\title{
A new mechanism for regional atmospheric chemistry modeling
}

\author{
William R. Stockwell, Frank Kirchner, and Michael Kuhn \\ Fraunhofer Institute for Atmospheric Environmental Research (IFU), Garmisch-Partenkirchen, Germany \\ Stephan Seefeld \\ Swiss Federal Institute for Environmental Science and Tcchnology (EAWAG), ETH Zürich, Switzerland
}

\begin{abstract}
A new gas-phase chemical mechanism for the modeling of regional atmospheric chemistry, the "Regional Atmospheric Chemistry Mechanism" (RACM) is presented. The mechanism is intended to be valid for remote to polluted conditions and from the Earth's surface through the upper troposphere. The RACM mechanism is based upon the earlier Regional Acid Deposition Model, version 2 (RADM2) mechanism [Stockwell et al., 1990] and the more detailed Euro-RADM mechanism [Stockwell and Kley, 1994]. The RACM mechanism includes rate constants and product yields from the most recent laboratory measurements, and it has been tested against environmental chamber data. A new condensed reaction mechanism is included for biogenic compounds: isoprene, $\alpha$-pinene, and d-limonene. The branching ratios for alkane decay were reevaluated, and in the revised mechanism the aldehyde to ketone ratios were significantly reduced. The relatively large amounts of nitrates resulting from the reactions of unbranched alkenes with $\mathrm{NO}_{3}$ are now included, and the production of $\mathrm{HO}$ from the ozonolysis of alkenes has a much greater yield. The aromatic chemistry has been revised through the use of new laboratory data. The yield of cresol production from aromatics was reduced, while the reactions of $\mathrm{HO}, \mathrm{NO}_{3}$, and $\mathrm{O}_{3}$ with unsaturated dicarbonyl species and unsaturated peroxynitrate are now included in the RACM mechanism. The peroxyacetyl nitrate chemistry and the organic peroxy radical-peroxy radical reactions were revised, and organic peroxy radical $+\mathrm{NO}_{3}$ reactions were added.
\end{abstract}

\section{Introduction}

The gas-phase chemical mechanism is one of the most important components of an atmospheric chemistry model. These models require high-quality gas-phase chemical mechanisms to calculate the concentrations of atmospheric chemical species. The concentrations of ozone and other air pollutants are determined by the emissions of nitrogen oxides and reactive organic species, gas- and aqueous-phase chemical reaction rates, deposition, and meteorological conditions.

There are several important mechanisms which are widely used for modeling the chemistry of the troposphere including the mechanism of Lurmann et al. [1986], the carbon bond IV mechanism of Gery et al. [1989], and the mechanism for the Regional Acid Deposition Modcl (RADM2) [Stockwell et al., 1990]. For example, the RADM2 mechanism is used in many photochemical transport/transformation atmospheric chemistry models to predict concentrations of oxidants and other air pollutants [Chang et al., 1991; Hass et al., 1995; Vogel et al., 1995].

Since these mechanisms have been published, much new laboratory work has become available [Le Bras, 1997; DeMore et al., 1994; Atkinson et al., 1992b; Atkinson, 1994]. The purpose of this paper is to present a completely revised version of the mechanism of Stockwell et al. [1990] and to show the effect of the revisions on calculated chemical concentrations. Since the purpose of the new mechanism is to describe atmospheric

Copyright 1997 by the American Geophysical Union.

Paper number 97JD00849.

0148-0227/97/97JD-00849\$09.00 chemistry on a regional scale, we have named it the "Regional Atmospheric Chemistry Mechanism" (RACM). The RACM mechanism was created to be capable of simulating the troposphere from the Earth's surface through the upper troposphere and to be valid for simulating remote to polluted urban conditions.

The mechanism includes 17 stable inorganic species, 4 inorganic intermediates, 32 stable organic species (4 of these are primarily of biogenic origin), and 24 organic intermediates (Table 1). The RACM mechanism includes 237 reactions (Table 2). The mechanism and its use are described in the subsequent text. The text is divided into section 2 on the inorganic chemistry and section 3 on the organic chemistry. The organic chemistry section includes a description of the aggregation procedures for emissions and the development of the chemistry for alkanes, carbonyls, alkenes, aromatics; the decomposition products of aromatics; and the primarily biogenic species, isoprene, $\alpha$-pinene, and d-limonene. The oxidation mechanism for isoprene, $\alpha$-pinene, and d-limonene is much more detailed and realistic than that in the RADM2 mechanism [Stockwell et al., 1990]. The aggregation factors for the organic emissions are given in Table 3. Sections 4 and 5 present comparisons of the mechanism with environmental chamber data and with the RADM2 mechanism, respectively. The treatment of peroxy radical reactions were described by Kirchner and Stockwell [1996a].

\section{Inorganic Chemistry}

Tropospheric inorganic chemistry is relatively well known. During the day the chemistry is driven by the photolysis of 
Table 1. RACM Mechanism Species List

\begin{tabular}{|c|c|c|c|c|}
\hline No. & Species & Definition & $\begin{array}{l}\text { Carbon } \\
\text { Number }\end{array}$ & $\begin{array}{l}\text { Molecular } \\
\text { Weight }\end{array}$ \\
\hline \multicolumn{5}{|c|}{ Stable Inorganic Compounds } \\
\hline & Oxidants & & & \\
\hline \multicolumn{5}{|c|}{ ozone } \\
\hline 2 & $\begin{array}{c}\mathrm{H}_{2} \mathrm{O}_{2} \\
\text { Nitrogenous }\end{array}$ & \multirow{2}{*}{\multicolumn{2}{|c|}{ hydrogen peroxide }} & \multirow[t]{2}{*}{34} \\
\hline & compounds & & & \\
\hline 3 & NO & \multicolumn{2}{|l|}{ nitric oxide } & 30 \\
\hline 4 & $\mathrm{NO}_{2}$ & \multicolumn{2}{|l|}{ nitrogen dioxide } & 46 \\
\hline 5 & $\mathrm{NO}_{3}$ & \multicolumn{2}{|l|}{ nitrogen trioxide } & 62 \\
\hline 6 & $\mathrm{~N}_{2} \mathrm{O}_{5}$ & \multicolumn{2}{|l|}{ dinitrogen pentoxide } & 108 \\
\hline 7 & HONO & \multicolumn{2}{|l|}{ nitrous acid } & 47 \\
\hline 8 & $\mathrm{HNO}_{3}$ & \multicolumn{2}{|l|}{ nitric acid } & 63 \\
\hline 9 & $\mathrm{HNO}_{4}$ & \multicolumn{2}{|l|}{ pernitric acid } & \multirow[t]{2}{*}{79} \\
\hline & $\begin{array}{l}\text { Sulfur } \\
\text { compounds }\end{array}$ & & & \\
\hline 10 & $\mathrm{SO}_{2}-\mathrm{T}_{2}$ & \multicolumn{2}{|l|}{ sulfur dioxide } & 64 \\
\hline 11 & SULF & \multicolumn{2}{|l|}{ sulfuric acid } & \multirow{2}{*}{98} \\
\hline & Carbon oxides & & & \\
\hline 12 & $\mathrm{CO}$ & \multicolumn{2}{|l|}{ carbon monoxide } & 28 \\
\hline 13 & $\mathrm{CO}_{2}$ & carbon dioxide & 1 & 44 \\
\hline & & Abundant Stable Species & & \\
\hline 14 & $\mathrm{~N}_{2}$ & nitrogen & & 28 \\
\hline 15 & $\mathrm{O}_{2}$ & oxygen & & 32 \\
\hline 16 & $\mathrm{H}_{2} \mathrm{O}$ & water & & 18 \\
\hline 17 & $\mathrm{H}_{2}$ & hydrogen & & 2 \\
\hline & & Inorganic Short-Lived Intermediates & & \\
\hline 18 & $\mathrm{O}^{3} \mathrm{P}$ & ground state oxygen atom, $\mathrm{O}\left({ }^{3} P\right)$ & & 16 \\
\hline 19 & $\mathrm{O}^{1} D$ & excited state oxygen atom, $\mathrm{O}\left({ }^{1} D\right)$ & & 16 \\
\hline & Odd hydrogen & & & \\
\hline 20 & $\mathrm{HO}$ & hydroxy radical & & 17 \\
\hline 21 & $\mathrm{HO}_{2}$ & hydroperoxy radical & & 33 \\
\hline & & Stable Organic Compounds & & \\
\hline & Alkanes & & & \\
\hline 22 & $\mathrm{CH}_{4}$ & methane & 1.0 & 16 \\
\hline 23 & ETH & ethane & 2.0 & 30 \\
\hline 24 & $\mathrm{HC} 3$ & $\begin{array}{l}\text { alkanes, alcohols, esters, and alkynes with HO rate } \\
\text { constant }(298 \mathrm{~K}, 1 \mathrm{~atm}) \text { less than } 3.4 \times 10^{-12} \mathrm{~cm}^{3} \mathrm{~s}^{-1}\end{array}$ & 2.9 & 44 \\
\hline 25 & HC5 & $\begin{array}{l}\text { alkanes, alcohols, esters, and alkynes with } \mathrm{HO} \text { rate } \\
\text { constant }(298 \mathrm{~K}, 1 \mathrm{~atm}) \text { between } 3.4 \times 10^{-12} \text { and } \\
6.8 \times 10^{-12} \mathrm{~cm}^{3} \mathrm{~s}^{-1}\end{array}$ & 4.8 & 72 \\
\hline 26 & $\mathrm{HC} 8$ & alkanes, alcohols, esters, and alkynes with $\mathrm{HO}$ rate & 7.9 & 114 \\
\hline & & $\begin{array}{l}\text { constant }(298 \mathrm{~K}, 1 \mathrm{~atm}) \text { greater than } 6.8 \times 10^{-12} \\
\mathrm{~cm}^{3} \mathrm{~s}^{-1}\end{array}$ & & \\
\hline & Alkenes & & & \\
\hline 27 & ETE & ethene & 2.0 & 28 \\
\hline 28 & OLT & terminal alkenes & 3.8 & 42 \\
\hline 29 & OLI & internal alkenes & 5.0 & 68 \\
\hline 30 & DIEN & butadiene and other anthropogenic dienes & 4.0 & 54 \\
\hline & $\begin{array}{c}\text { Stable biogenic } \\
\text { alkenes }\end{array}$ & & & \\
\hline 31 & ISO & isoprene & 5.0 & 68 \\
\hline 32 & API & $\begin{array}{l}\alpha \text {-pinene and other cyclic terpenes with one double } \\
\text { bond }\end{array}$ & 10.0 & 136 \\
\hline 33 & LIM & d-limonene and other cyclic diene-terpenes & 10.0 & 136 \\
\hline & Aromatics & & & \\
\hline 34 & TOL & toluene and less reactive aromatics & 7.1 & 92 \\
\hline 35 & XYL & xylene and more reactive aromatics & 8.9 & 106 \\
\hline 36 & CSL & cresol and other hydroxy substituted aromatics & 6.6 & 108 \\
\hline & Carbonyls & & & \\
\hline 37 & $\mathrm{HCHO}$ & formaldehyde & 1.0 & 30 \\
\hline 38 & ALD & acetaldehyde and higher aldehydes & 2.4 & 44 \\
\hline 39 & KET & ketones & 3.5 & 72 \\
\hline 40 & GLY & glyoxal & 2.0 & 58 \\
\hline 41 & MGLY & methylglyoxal and other $\alpha$-carbonyl aldehydes & 3.0 & 72 \\
\hline 42 & DCB & unsaturated dicarbonyls & 4.2 & 87 \\
\hline 43 & MACR & methacrolein and other unsaturated monoaldehydes & 4.0 & 70 \\
\hline 44 & UDD & unsaturated dihydroxy dicarbonyl & 4.2 & 119 \\
\hline 45 & HKET & hydroxy ketone & 3.0 & 74 \\
\hline
\end{tabular}


Table 1. (continued)

\begin{tabular}{|c|c|c|c|c|}
\hline No. & Spccies & Definition & $\begin{array}{l}\text { Carbon } \\
\text { Numbcr }\end{array}$ & $\begin{array}{l}\text { Molecular } \\
\text { Weight }\end{array}$ \\
\hline & \multicolumn{4}{|l|}{ Organic nitrogen } \\
\hline 46 & ONIT & organic nitrate & 4.0 & 119 \\
\hline 47 & PAN & peroxyacetyl nitrate and higher saturated PANs & 2.0 & 121 \\
\hline 48 & TPAN & unsaturated PANs & 4.0 & 147 \\
\hline \multicolumn{5}{|c|}{ Organic peroxides } \\
\hline 49 & OP1 & methyl hydrogen peroxide & 1.0 & 48 \\
\hline 50 & OP2 & higher organic peroxides & 2.0 & 62 \\
\hline 51 & PAA & peroxyacetic acid and higher analogs & 2.0 & 76 \\
\hline \multicolumn{5}{|c|}{ Organic acids } \\
\hline 52 & ORA1 & formic acid & 1.0 & 46 \\
\hline 53 & ORA2 & acetic acid and higher acids & 2.0 & 60 \\
\hline \multicolumn{5}{|c|}{ Organic Short-Lived Intermediates } \\
\hline \multicolumn{5}{|c|}{$\begin{array}{l}\text { Peroxy radicals from } \\
\quad \text { alkanes }\end{array}$} \\
\hline 54 & $\mathrm{MO}_{2}$ & methyl peroxy radical & 1.0 & 47 \\
\hline 55 & ETHP & peroxy radical formed from ETH & 2.0 & 61 \\
\hline 56 & HC3P & peroxy radical formed from $\mathrm{HC} 3$ & 2.9 & 75 \\
\hline 57 & HC5P & peroxy radical formed from HC5 & 4.8 & 103 \\
\hline 58 & $\mathrm{HC} 8 \mathrm{P}$ & peroxy radical formed from $\mathrm{HC} 8$ & 7.9 & 145 \\
\hline \multicolumn{5}{|c|}{$\begin{array}{l}\text { Peroxy radicals from } \\
\text { alkenes. }\end{array}$} \\
\hline 59 & ETEP & peroxy radicals formed from ETE & 2.0 & 77 \\
\hline 60 & OLTP & peroxy radicals formed from OLT & 3.8 & 91 \\
\hline 61 & OLIP & peroxy radicals formed from OLI & 4.8 & 117 \\
\hline \multicolumn{5}{|c|}{$\begin{array}{l}\text { Peroxy radicals from } \\
\text { biogenic alkenes }\end{array}$} \\
\hline 62 & ISOP & peroxy radicals formed from ISO and DIEN & 5.0 & 117 \\
\hline 63 & APIP & peroxy radicals formed from API & 10.0 & 185 \\
\hline 64 & LIMP & peroxy radicals formed from LIM & 10.0 & 185 \\
\hline \multicolumn{5}{|c|}{$\begin{array}{l}\text { Radicals produced } \\
\quad \text { from aromatics }\end{array}$} \\
\hline 65 & PHO & phenoxy radical and similar radicals & 6.6 & 107 \\
\hline 66 & ADDT & aromatic-HO adduct from TOL & 7.1 & 109 \\
\hline 67 & ADDX & aromatic-HO adduct from XYL & 8.9 & 123 \\
\hline 68 & $\mathrm{ADDC}$ & aromatic-HO adduct from CSL & 6.6 & 125 \\
\hline 69 & TOLP & peroxy radicals formed from TOL & 7.1 & 141 \\
\hline 70 & XYLP & peroxy radicals formed from XYL & 8.9 & 155 \\
\hline 71 & CSLP & pcroxy radicals formed from CSL & 6.6 & 157 \\
\hline \multicolumn{5}{|c|}{$\begin{array}{l}\text { Peroxy radicals with } \\
\text { carbonyl groups }\end{array}$} \\
\hline 72 & $\mathrm{ACO}_{3}$ & $\begin{array}{l}\text { acetyl peroxy and higher saturated acyl peroxy } \\
\text { radicals }\end{array}$ & 2.0 & 75 \\
\hline 73 & $\mathrm{TCO}_{3}$ & unsatured acyl peroxy radicals & 4.0 & 115 \\
\hline 74 & KETP & peroxy radicals formed from KET & 3.9 & 103 \\
\hline \multicolumn{5}{|c|}{$\begin{array}{l}\text { Other peroxy } \\
\text { radicals }\end{array}$} \\
\hline 75 & OLNN & $\mathrm{NO}_{3}$-alkene adduct reacting to form carbonitrates & $+\mathrm{HO}_{2} 3.0$ & 136 \\
\hline 76 & OLND & $\mathrm{NO}_{3}$-akene adduct reacting via decomposition & 3.0 & 136 \\
\hline 77 & $\mathrm{XO}_{2}$ & accounts for additional NO to $\mathrm{NO}_{2}$ conversions & $\cdots$ & $\cdots$ \\
\hline
\end{tabular}

ozone and nitrogen dioxide [Warneck, 1988]. The photolysis of ozone produces excited oxygen atoms, $O\left({ }^{1} D\right)$, and a fraction of these react with $\mathrm{H}_{2} \mathrm{O}$ to produce the hydroxyl radical (HO). HO reacts with inorganic and organic species to oxidize them. Many of these reactions produce $\mathrm{HO}_{2}$ or organic peroxy radicals which either react with $\mathrm{NO}$ to convert it to $\mathrm{NO}_{2}$ or (under low $\mathrm{NO}_{x}$ conditions) react to produce hydroperoxides. The conversion of $\mathrm{NO}$ to $\mathrm{NO}_{2}$ and the subsequent photolysis of $\mathrm{NO}_{2}$ produces more ozone. Ozone and $\mathrm{NO}_{2}$ react to form nitrate radical $\left(\mathrm{NO}_{3}\right)$ which is a very important reactive species during the nighttime [Morris and Niki, 1974; Japar and Niki, 1975; Platt et al., 1980]. The RACM mechanism has a reasonably complete set of inorganic reactions. The inorganic rate constants were set to the values recommended by DeMore et al.
[1994], and most of these changes from RADM2 were relatively small.

The cross sections and quantum yields for the photolysis of the inorganic species are taken from DeMore et al. [1994]. For $\mathrm{NO}_{3}$, DeMore et al. [1994] recommended the data from the review of Wayne et al. [1991]. Comparing the revised photolysis frequencies to the previous RADM2 values, there is an increase of $15-20 \%$ in the photolysis frequencies for HONO and a similar percent increase for both $\mathrm{NO}_{3}$ photolysis channels. Larger relative changes occur for the relatively low photolysis frequencies of $\mathrm{HNO}_{3}$ and $\mathrm{HO}_{2} \mathrm{NO}_{2}$. The photolysis frequencies for $\mathrm{NO}_{2}$ and $\mathrm{O}_{3}$ remained nearly unchanged after the updates.

Several inorganic reactions were added to the RACM mech- 
Table 2a. The RACM Mechanism: Photolysis Reactions

\begin{tabular}{|c|c|c|c|c|}
\hline $\begin{array}{l}\text { Reaction } \\
\text { No. }\end{array}$ & Reaction & $\begin{array}{l}\text { Photolysis } \\
\text { Frequency, } \\
\mathrm{s}^{-1}\end{array}$ & Cross Section & Quantum Yield \\
\hline (R1) & $\mathrm{NO}_{2} \rightarrow \mathrm{O}^{3} \mathrm{P}+\mathrm{NO}$ & $7.50 \times 10^{-3}$ & DeMore et al. [1994] & DeMore et al. [1994] \\
\hline (R2) & $\mathrm{O}_{3} \rightarrow \mathrm{O}^{1} \mathrm{D}+\mathrm{O}_{2}$ & $1.62 \times 10^{-5}$ & DeMore et al. [1994] & DeMore et al. [1994] \\
\hline (R3) & $\mathrm{O}_{3} \rightarrow \mathrm{O}^{3} \mathrm{P}+\mathrm{O}_{2}$ & $4.17 \times 10^{-4}$ & DeMore et al. [1994] & $\begin{array}{l}\text { total yield of } \mathrm{O}^{1} D \text { and } \mathrm{O}^{3} P \\
\text { assumed to be unity }\end{array}$ \\
\hline (R4) & $\mathrm{HONO} \rightarrow \mathrm{HO}+\mathrm{NO}$ & $1.63 \times 10^{-3}$ & DeMore et al. [1994] & DeMore et al. [1994] \\
\hline (R5) & $\mathrm{HNO}_{3} \rightarrow \mathrm{HO}+\mathrm{NO}_{2}$ & $4.50 \times 10^{-7}$ & DeMore et al. [1994] & assumed to be unity \\
\hline (R6) & $\mathrm{HNO}_{4} \rightarrow 0.65 \mathrm{HO}_{2}+0.65 \mathrm{NO}_{2}+0.35 \mathrm{HO}+0.35 \mathrm{NO}_{3}$ & $3.17 \times 10^{-6}$ & DeMore et al. [1994] & assumed to be unity \\
\hline (R7) & $\mathrm{NO}_{3} \rightarrow \mathrm{NO}+\mathrm{O}_{2}$ & $2.33 \times 10^{-2}$ & Wayne et al. [1991] & Wayne et al. [1991] \\
\hline (R8) & $\mathrm{NO}_{3} \rightarrow \mathrm{NO}_{2}+\mathrm{O}^{3} \mathrm{P}$ & $1.87 \times 10^{-1}$ & Wayne et al. [1991] & Wayne et al. [1991] \\
\hline (R9) & $\mathrm{H}_{2} \mathrm{O}_{2} \rightarrow \mathrm{HO}+\mathrm{HO}$ & $6.00 \times 10^{-6}$ & DeMore et al. [1994] & assumed to be unity \\
\hline (R10) & $\mathrm{HCHO} \rightarrow \mathrm{H}_{2}+\mathrm{CO}$ & $3.50 \times 10^{-5}$ & $\begin{array}{l}\text { wavelengths }<300 \mathrm{~nm}: \\
\text { Moortgat et al. }[1980]\end{array}$ & $\begin{array}{l}\text { wavelengths }<300 \mathrm{~nm}: \\
\text { Atkinson et al. }[1994]^{\mathrm{b}}\end{array}$ \\
\hline & & & $\begin{array}{l}\text { wavelengths }>300 \mathrm{~nm}: \\
\text { Cantrell et al. }[1990]\end{array}$ & $\begin{array}{l}\text { wavelengths }>300 \mathrm{~nm}: \\
\text { DeMore et al. }[1994]^{\mathrm{b}}\end{array}$ \\
\hline (R11) & $\mathrm{HCHO} \rightarrow 2 \mathrm{HO}_{2}+\mathrm{CO}$ & $2.17 \times 10^{-5}$ & same references as (R10) & same references as $(\mathrm{R} 10)^{\mathrm{b}}$ \\
\hline (R12) & $\mathrm{ALD} \rightarrow \mathrm{MO}_{2}+\mathrm{HO}_{2}+\mathrm{CO}$ & $3.67 \times 10^{-6}$ & Martinez et al. [1992] & Atkinson $[1994]^{\mathrm{b}}$ \\
\hline (R13) & $\mathrm{OP} 1 \rightarrow \mathrm{HCHO}+\mathrm{HO}_{2}+\mathrm{HO}$ & $4.17 \times 10^{-6}$ & DeMore et al. [1994] & DeMore et al. [1994] \\
\hline (R14) & $\mathrm{OP} 2 \rightarrow \mathrm{ALD}+\mathrm{HO}_{2}+\mathrm{HO}$ & $4.17 \times 10^{-6}$ & same as (R13) & same as (R13) \\
\hline (R15) & $\mathrm{PAA} \rightarrow \mathrm{MO}_{2}+\mathrm{HO}$ & $1.57 \times 10^{-6}$ & $\begin{array}{l}\mathrm{H}_{2} \mathrm{O}_{2} \text { cross sections } \\
\quad \text { scaled by } 0.28 ; \text { Giguere } \\
\text { and Olmos }[1956]\end{array}$ & assumed to be unity \\
\hline (R16) & $\mathrm{KET} \rightarrow \mathrm{ETHP}+\mathrm{ACO}_{3}$ & $6.67 \times 10^{-7}$ & Martinez et al. [1992] & Atkinson [1994] \\
\hline (R17) & $\mathrm{GLY} \rightarrow 0.13 \mathrm{HCHO}+1.87 \mathrm{CO}+0.87 \mathrm{H}_{2}$ & $5.83 \times 10^{-5}$ & Atkinson et al. [1992b] & $\begin{array}{l}\text { wavelengths < }<340: 0.0 \\
\text { wavclengths }>340: 0.029 \\
\text { Atkinson et }\end{array}$ \\
\hline (R18) & $\begin{aligned} \mathrm{GLY} \rightarrow & 0.45 \mathrm{HCHO}+1.55 \mathrm{CO}+0.80 \mathrm{HO}_{2} \\
& +0.15 \mathrm{H}_{2}\end{aligned}$ & $2.00 \times 10^{-5}$ & Atkinson et al. [1992b] & $\begin{array}{l}\text { wavelengths <340: } 0.4 \\
\text { wavelengths }>340: 0.0 \\
\text { Atkinson et al. }[1992 \mathrm{~b}]\end{array}$ \\
\hline (R19) & $\mathrm{MGLY} \rightarrow \mathrm{CO}+\mathrm{HO}_{2}+\mathrm{ACO}_{3}$ & $9.33 \times 10^{-5}$ & $\begin{array}{l}\text { Atkinson [1994] and } \\
\text { Staffelbach et al. }[1995]\end{array}$ & $\begin{array}{l}\text { estimated from Koch and } \\
\text { Moortgat [1996] }\end{array}$ \\
\hline (R20) & $\mathrm{DCB} \rightarrow \mathrm{TCO}_{3}+\mathrm{HO}_{2}$ & $4.33 \times 10^{-4}$ & Stockwell et al. [1990] & Stockwell et al. [1990] \\
\hline (R21) & $\mathrm{ONIT} \rightarrow 0.20 \mathrm{ALD}+0.80 \mathrm{KET}+\mathrm{HO}_{2}+\mathrm{NO}_{2}$ & $2.17 \times 10^{-6}$ & $\begin{array}{l}\text { assumed to be mixture of } \\
20 \% \text { n-propyl nitrate } \\
\text { and } 80 \% \text { i-propyl } \\
\text { nitrate; Atkinson [1994] }\end{array}$ & Atkinson [1994] \\
\hline$(\mathrm{R} 22)$ & $\mathrm{MACR} \rightarrow \mathrm{CO}+\mathrm{HCHO}+\mathrm{HO}_{2}+\mathrm{ACO}_{3}$ & $1.33 \times 10^{-6}$ & $\begin{array}{l}\text { Gardner et al. [1987] } \\
\text { assumed equal to } \\
\text { acrolein }\end{array}$ & Gardner et al. [1987] \\
\hline (R23) & $\mathrm{HKET} \rightarrow \mathrm{HCHO}+\mathrm{HO}_{2}+\mathrm{ACO}_{3}$ & $6.67 \times 10^{-7}$ & same as (R16) & same as (R16) \\
\hline
\end{tabular}

a Typical photolysis frequencies are given for solar zenith angle $40^{\circ}$, June 21 , summer surface $40^{\circ}$ northern latitude.

bressure dependences given by $\varphi(\lambda, P)=\varphi(\lambda, 1 \mathrm{~atm}) /\{\varphi(\lambda, 1 \mathrm{~atm})+[1-\varphi(\lambda, 1 \mathrm{~atm})] \times P\}$, where $P$ is in atmospheres.

anism. The reaction of $\mathrm{O}\left({ }^{3} P\right)$ with nitrogen oxides, especially the reaction of $\mathrm{O}\left({ }^{3} P\right)$ with $\mathrm{NO}_{2}$, can be important in the upper atmosphere [Warneck, 1988]:

$$
\begin{gathered}
\mathrm{O}\left({ }^{3} P\right)+\mathrm{NO}_{2} \rightarrow \mathrm{NO}+\mathrm{O}_{2} \\
\mathrm{O}\left({ }^{3} P\right)+\mathrm{NO}_{2}(+\mathrm{M}) \rightarrow \mathrm{NO}_{3}(+\mathrm{M}) \\
\mathrm{O}\left({ }^{3} P\right)+\mathrm{NO}(+\mathrm{M}) \rightarrow \mathrm{NO}_{2}(+\mathrm{M})
\end{gathered}
$$

In the original RADM2 mechanism the reaction of $\mathrm{O}\left({ }^{3} P\right)$ with $\mathrm{NO}_{2}$ produced only $\mathrm{NO}$; however, $\mathrm{O}\left({ }^{3} P\right)$ can also add to $\mathrm{NO}_{2}$ to produce $\mathrm{NO}_{3}$ [DeMore et al., 1994]. Both reactions are now included although rapid photolysis of $\mathrm{NO}_{3}$ would be expected to limit the importance of (2).

The reaction of $\mathrm{O}\left({ }^{3} P\right)$ with ozone was added because it is important in the upper atmosphere [Warneck, 1988]:

$$
\mathrm{O}\left({ }^{3} \mathrm{P}\right)+\mathrm{O}_{3} \rightarrow 2 \mathrm{O}_{2}
$$

The reaction of $\mathrm{HO}$ with $\mathrm{I}_{2}$ is a significant loss reaction for $\mathrm{HO}$ in the remote atmosphere:

$$
\mathrm{HO}+\mathrm{H}_{2}+\left(\mathrm{O}_{2}\right) \rightarrow \mathrm{HO}_{2}+\mathrm{H}_{2} \mathrm{O}
$$

The rate constant for (5) at $298 \mathrm{~K}$ is $6.7 \times 10^{-15} \mathrm{~cm}^{3} \mathrm{~s}^{-1}$ [DeMore et al., 1994] which is about equal to the rate constant for the $\mathrm{HO}+\mathrm{CH}_{4}$ reaction, $6.9 \times 10^{-15} \mathrm{~cm}^{-3} \mathrm{~s}^{-1}$ [Atkinson, 1994]. If the surface concentrations for $\mathrm{H}_{2}$ and $\mathrm{CH}_{4}$ are assumed to be 500 and 1700 ppbv, respectively, then the rate of the reaction of $\mathrm{HO}$ with $\mathrm{H}_{2}$ is about $30 \%$ that of the $\mathrm{HO}+$ $\mathrm{CH}_{4}$ reaction.

For completeness, the reaction of $\mathrm{HO}$ with nitrous acid (6) was added. The rate constant for this reaction is $4.86 \times 10^{-12}$ $\mathrm{cm}^{-3} \mathrm{~s}^{-1}$ at $298 \mathrm{~K}$. The rate of the $\mathrm{HONO}+\mathrm{HO}$ is about $2.4 \%$ of the $\mathrm{HONO}$ photolysis reaction if an $\mathrm{HO}$ concentration of $1 \times 10^{7} \mathrm{~cm}^{-3}$ and a HONO photolysis frequency of $2 \times$ $10^{-3} \mathrm{~s}$ are assumed:

$$
\mathrm{HO}+\mathrm{HONO} \rightarrow \mathrm{NO}_{2}+\mathrm{H}_{2} \mathrm{O}
$$

The reactions of $\mathrm{NO}_{3}$ are very important for nighttime atmospheric chemistry. The $\mathrm{NO}_{3}$ self-reaction (7) was added for atmospheric conditions with very high $\mathrm{NO}_{x}$ and $\mathrm{O}_{3}$ concentrations during the nighttime. For example, for $\mathrm{NO}_{3}$ concentration of 300 parts per trillion (ppt) [Platt et al., 1980] and a NO 
Table 2b. The RACM Mechanism

\begin{tabular}{|c|c|c|c|c|c|}
\hline $\begin{array}{l}\text { Reaction } \\
\text { No. }\end{array}$ & Reaction & $\begin{array}{c}A, \\
\mathrm{~cm}^{3} \mathrm{~s}^{-1}\end{array}$ & $\begin{array}{c}E / R \\
\mathbf{K}\end{array}$ & $k^{\mathrm{a}}$ & Note \\
\hline \multicolumn{6}{|c|}{ Inorganic Reactions } \\
\hline (R24) & $\mathrm{O}^{3} \mathrm{P}+\mathrm{O}_{2} \rightarrow \mathrm{O}_{3}$ & Table $2 \mathrm{f}$ & & $1.50 \times 10^{-14}$ & 1 \\
\hline (R25) & $\mathrm{O}^{3} \mathrm{P}+\mathrm{O}_{3} \rightarrow 2 \mathrm{O}_{2}$ & $8.00 \times 10^{-12}$ & 2060 & $7.96 \times 10^{-15}$ & 1 \\
\hline (R26) & $\mathrm{O}^{1} D+\mathrm{N}_{2} \rightarrow \mathrm{O}^{3} P+\mathrm{N}_{2}$ & $1.80 \times 10^{-11}$ & -110 & $2.60 \times 10^{-11}$ & 1 \\
\hline (R27) & $\mathrm{O}^{1} D+\mathrm{O}_{2} \rightarrow \mathrm{O}^{3} \mathrm{P}+\mathrm{O}_{2}$ & $3.20 \times 10^{-11}$ & -70 & $4.05 \times 10^{-11}$ & 1 \\
\hline (R28) & $\mathrm{O}^{1} D+\mathrm{H}_{2} \mathrm{O} \rightarrow \mathrm{HO}+\mathrm{HO}$ & $2.20 \times 10^{-10}$ & & $2.20 \times 10^{-10}$ & 1 \\
\hline (R29) & $\mathrm{O}_{3}+\mathrm{HO} \rightarrow \mathrm{HO}_{2}+\mathrm{O}_{2}$ & $1.60 \times 10^{-12}$ & 940 & $6.83 \times 10^{-14}$ & 1 \\
\hline (R30) & $\mathrm{O}_{3}+\mathrm{HO}_{2} \rightarrow \mathrm{HO}+2 \mathrm{O}_{2}$ & $1.10 \times 10^{-14}$ & 500 & $2.05 \times 10^{-15}$ & 1 \\
\hline (R31) & $\mathrm{HO}+\mathrm{HO}_{2} \rightarrow \mathrm{H}_{2} \mathrm{O}+\mathrm{O}_{2}$ & $4.80 \times 10^{-11}$ & -250 & $1.11 \times 10^{-10}$ & 1 \\
\hline (R32) & $\mathrm{H}_{2} \mathrm{O}_{2}+\mathrm{HO} \rightarrow \mathrm{HO}_{2}+\mathrm{H}_{2} \mathrm{O}$ & $2.90 \times 10^{-12}$ & 160 & $1.70 \times 10^{-12}$ & 1 \\
\hline (R33) & $\mathrm{HO}_{2}+\mathrm{HO}_{2} \rightarrow \mathrm{H}_{2} \mathrm{O}_{2}+\mathrm{O}_{2}$ & Table $2 \mathrm{f}$ & & $2.92 \times 10^{-12}$ & 1 \\
\hline (R34) & $\mathrm{HO}_{2}+\mathrm{HO}_{2}+\mathrm{H}_{2} \mathrm{O} \rightarrow \mathrm{H}_{2} \mathrm{O}_{2}+\mathrm{O}_{2}+\mathrm{H}_{2} \mathrm{O}$ & Table $2 \mathrm{f}$ & & $6.58 \times 10^{-30}$ & 1 \\
\hline (R35) & $\mathrm{O}^{3} \mathrm{P}+\mathrm{NO} \rightarrow \mathrm{NO}_{2}$ & Table $2 \mathrm{~d}$ & & $1.66 \times 10^{-12}$ & 1 \\
\hline (R36) & $\mathrm{O}^{3} \mathrm{P}+\mathrm{NO}_{2} \rightarrow \mathrm{NO}+\mathrm{O}_{2}$ & $6.50 \times 10^{-12}$ & -120 & $9.72 \times 10^{-12}$ & 1 \\
\hline (R37) & $\mathrm{O}^{3} \mathrm{P}+\mathrm{NO}_{2} \rightarrow \mathrm{NO}_{3}$ & Table $2 \mathrm{~d}$ & & $1.58 \times 10^{-12}$ & 1 \\
\hline (R38) & $\mathrm{HO}+\mathrm{NO} \rightarrow \mathrm{HONO}$ & Table 2d & & $4.87 \times 10^{-12}$ & 1 \\
\hline (R39) & $\mathrm{HO}+\mathrm{NO}_{2} \rightarrow \mathrm{HNO}_{3}$ & Table $2 \mathrm{~d}$ & & $1.15 \times 10^{-11}$ & 1 \\
\hline (R40) & $\mathrm{HO}+\mathrm{NO}_{3} \rightarrow \mathrm{NO}_{2}+\mathrm{HO}_{2}$ & $2.20 \times 10^{-11}$ & & $2.20 \times 10^{-11}$ & 1 \\
\hline (R41) & $\mathrm{HO}_{2}+\mathrm{NO} \rightarrow \mathrm{NO}_{2}+\mathrm{HO}$ & $3.70 \times 10^{-12}$ & -250 & $8.56 \times 10^{-12}$ & 1 \\
\hline (R42) & $\mathrm{HO}_{2}+\mathrm{NO}_{2} \rightarrow \mathrm{HNO}_{4}$ & Table 2d & & $1.39 \times 10^{-12}$ & 1 \\
\hline (R43) & $\mathrm{HNO}_{4} \rightarrow \mathrm{HO}_{2}+\mathrm{NO}_{2}$ & Table $2 \mathrm{e}$ & & $8.62 \times 10^{-2}$ & 1 \\
\hline (R44) & $\mathrm{HO}_{2}+\mathrm{NO}_{3} \rightarrow 0.3 \mathrm{HNO}_{3}+0.7 \mathrm{NO}_{2}+0.7 \mathrm{HO}+\mathrm{O}_{2}$ & $3.50 \times 10^{-12}$ & & $3.50 \times 10^{-12}$ & 1,2 \\
\hline (R45) & $\mathrm{HO}+\mathrm{HONO} \rightarrow \mathrm{NO}_{2}+\mathrm{H}_{2} \mathrm{O}$ & $1.80 \times 10^{-11}$ & 390 & $4.86 \times 10^{-12}$ & 1 \\
\hline$(\mathrm{R} 46)$ & $\mathrm{HO}+\mathrm{HNO}_{3} \rightarrow \mathrm{NO}_{3}+\mathrm{H}_{2} \mathrm{O}$ & Table $2 \mathrm{f}$ & & $1.47 \times 10^{-13}$ & 1 \\
\hline (R47) & $\mathrm{HO}+\mathrm{HNO}_{4} \rightarrow \mathrm{NO}_{2}+\mathrm{O}_{2}+\mathrm{H}_{2} \mathrm{O}$ & $1.30 \times 10^{-12}$ & -380 & $4.65 \times 10^{-12}$ & 1,3 \\
\hline (R48) & $\mathrm{O}_{3}+\mathrm{NO} \rightarrow \mathrm{NO}_{2}+\mathrm{O}_{2}$ & $2.00 \times 10^{-12}$ & 1400 & $1.82 \times 10^{-14}$ & 1 \\
\hline (R49) & $\mathrm{O}_{3}+\mathrm{NO}_{2} \rightarrow \mathrm{NO}_{3}+\mathrm{O}_{2}$ & $1.20 \times 10^{-13}$ & 2450 & $3.23 \times 10^{-17}$ & 1 \\
\hline$(\mathrm{R} 50)$ & $\mathrm{NO}+\mathrm{NO}+\mathrm{O}_{2} \rightarrow \mathrm{NO}_{2}+\mathrm{NO}_{2}$ & $3.30 \times 10^{-39}$ & -530 & $1.95 \times 10^{-38}$ & 4 \\
\hline (R51) & $\mathrm{NO}_{3}+\mathrm{NO} \rightarrow \mathrm{NO}_{2}+\mathrm{NO}_{2}$ & $1.50 \times 10^{-11}$ & -170 & $2.65 \times 10^{-11}$ & 1 \\
\hline (R52) & $\mathrm{NO}_{3}+\mathrm{NO}_{2} \rightarrow \mathrm{NO}+\mathrm{NO}_{2}+\mathrm{O}_{2}$ & $4.50 \times 10^{-14}$ & 1260 & $6.56 \times 10^{-16}$ & 1 \\
\hline (R53) & $\mathrm{NO}_{3}+\mathrm{NO}_{2} \rightarrow \mathrm{N}_{2} \mathrm{O}_{5}$ & Table 2d & & $1.27 \times 10^{-12}$ & 1 \\
\hline (R54) & $\mathrm{N}_{2} \mathrm{O}_{5} \rightarrow \mathrm{NO}_{2}+\mathrm{NO}_{3}$ & Table 2e & & $4.36 \times 10^{-2}$ & 1 \\
\hline (R55) & $\mathrm{NO}_{3}+\mathrm{NO}_{3} \rightarrow \mathrm{NO}_{2}+\mathrm{NO}_{2}+\mathrm{O}_{2}$ & $8.50 \times 10^{-13}$ & 2450 & $2.29 \times 10^{-16}$ & 1 \\
\hline (R56) & $\mathrm{HO}+\mathrm{H}_{2} \rightarrow \mathrm{H}_{2} \mathrm{O}+\mathrm{HO}_{2}$ & $5.50 \times 10^{-12}$ & 2000 & $6.69 \times 10^{-15}$ & 1 \\
\hline (R57) & $\mathrm{HO}+\mathrm{SO}_{2} \rightarrow \mathrm{SULF}+\mathrm{HO}_{2}$ & Table 2d & & $8.89 \times 10^{-13}$ & 1 \\
\hline (R58) & $\mathrm{CO}+\mathrm{HO} \rightarrow \mathrm{HO}_{2}+\mathrm{CO}_{2}$ & Table $2 \mathrm{f}$ & & $2.40 \times 10^{-13}$ & 1 \\
\hline \multicolumn{6}{|c|}{$O^{3} P+$ Organic Compound } \\
\hline (R59) & $\begin{array}{l}\mathrm{ISO}+\mathrm{O}^{3} \mathrm{P} \rightarrow 0.86 \mathrm{OLT}+0.05 \mathrm{HCHO}+0.02 \mathrm{HO}+0.01 \mathrm{CO}+0.13 \\
\mathrm{DCB}+0.28 \mathrm{HO}+0.15 \mathrm{XO}\end{array}$ & $6.00 \times 10^{-11}$ & & $6.00 \times 10^{-11}$ & 5 \\
\hline (R60) & $\mathrm{MACR}+\mathrm{O}^{3} \mathrm{P} \rightarrow \mathrm{ALD}$ & $1.59 \times 10^{-11}$ & -13 & $1.66 \times 10^{-11}$ & 6 \\
\hline \multicolumn{6}{|c|}{$H O+$ Organic Compounds } \\
\hline (R61) & $\mathrm{CH}_{4}+\mathrm{HO} \rightarrow \mathrm{MO}_{2}+\mathrm{H}_{2} \mathrm{O}$ & Table $2 \mathrm{c}$ & & $6.86 \times 10^{-15}$ & 7 \\
\hline (R62) & $\mathrm{ETH}+\mathrm{HO} \rightarrow \mathrm{ETHP}+\mathrm{H}_{2} \mathrm{O}$ & Table $2 \mathrm{c}$ & & $2.57 \times 10^{-13}$ & 7 \\
\hline (R63) & $\begin{array}{l}\mathrm{HC} 3+\mathrm{HO} \rightarrow 0.583 \mathrm{HC} 3 \mathrm{P}+0.381 \mathrm{HO}_{2}+0.335 \mathrm{ALD}+0.036 \mathrm{ORA} 1 \\
+0.036 \mathrm{CO}+0.036 \mathrm{GLY}+0.036 \mathrm{HO}+0.010 \mathrm{HCHO}+\mathrm{H}_{2} \mathrm{O}\end{array}$ & $5.26 \times 10^{-12}$ & 260 & $2.20 \times 10^{-12}$ & 8 \\
\hline (R64) & $\mathrm{HC} 5+\mathrm{HO} \rightarrow 0.75 \mathrm{HC} 5 \mathrm{P}+0.25 \mathrm{KET}+0.25 \mathrm{HO}_{2}+\mathrm{H}_{2} \mathrm{O}$ & $8.02 \times 10^{-12}$ & 155 & $4.77 \times 10^{-12}$ & 8 \\
\hline (R65) & $\begin{array}{l}\mathrm{HC} 8+\mathrm{HO} \rightarrow 0.951 \mathrm{HC} 8 \mathrm{P}+0.025 \mathrm{ALD}+0.024 \mathrm{HKET}+0.049 \mathrm{HO}_{2} \\
+\mathrm{H}_{2} \mathrm{O}\end{array}$ & $1.64 \times 10^{-11}$ & 125 & $1.08 \times 10^{-11}$ & 8 \\
\hline (R66) & $\mathrm{ETE}+\mathrm{HO} \rightarrow \mathrm{ETEP}$ & $1.96 \times 10^{-12}$ & -438 & $8.52 \times 10^{-12}$ & 7 \\
\hline (R67) & $\mathrm{OLT}+\mathrm{HO} \rightarrow \mathrm{OLTP}$ & $5.72 \times 10^{-12}$ & -500 & $3.06 \times 10^{-11}$ & 8 \\
\hline (R68) & $\mathrm{OLI}+\mathrm{HO} \rightarrow$ OLIP & $1.33 \times 10^{-11}$ & -500 & $7.12 \times 10^{-11}$ & 8 \\
\hline (R69) & $\mathrm{DIEN}+\mathrm{HO} \rightarrow \mathrm{ISOP}$ & $1.48 \times 10^{-11}$ & -448 & $6.65 \times 10^{-11}$ & 7 \\
\hline (R70) & ISO $+\mathrm{HO} \rightarrow$ ISOP & $2.54 \times 10^{-11}$ & -410 & $1.01 \times 10^{-10}$ & 7 \\
\hline (R71) & $\mathrm{API}+\mathrm{HO} \rightarrow \mathrm{APIP}$ & $1.21 \times 10^{-11}$ & -444 & $5.37 \times 10^{-11}$ & 7 \\
\hline (R72) & LIM + HO $\rightarrow$ LIMP & $1.70 \times 10^{-10}$ & & $1.71 \times 10^{-10}$ & 7 \\
\hline (R73) & $\mathrm{TOL}+\mathrm{HO} \rightarrow 0.90 \mathrm{ADDT}+0.10 \mathrm{XO}_{2}+0.10 \mathrm{HO}_{2}$ & $1.81 \times 10^{-12}$ & -355 & $5.96 \times 10^{-12}$ & 7 \\
\hline (R74) & $\mathrm{XYL}+\mathrm{HO} \rightarrow 0.90 \mathrm{ADDX}+0.10 \mathrm{XO}_{2}+0.10 \mathrm{HO}_{2}$ & $7.30 \times 10^{-12}$ & -355 & $2.40 \times 10^{-11}$ & 7,10 \\
\hline$(\mathrm{R} 75)$ & $\mathrm{CSL}+\mathrm{HO} \rightarrow 0.85 \mathrm{ADDC}+0.10 \mathrm{PHO}+0.05 \mathrm{HO}_{2}+0.05 \mathrm{XO}_{2}$ & $6.00 \times 10^{-11}$ & & $6.00 \times 10^{-11}$ & 7,11 \\
\hline (R76) & $\mathrm{HCHO}+\mathrm{HO} \rightarrow \mathrm{HO}_{2}+\mathrm{CO}+\mathrm{H}_{2} \mathrm{O}$ & $1.00 \times 10^{-11}$ & & $1.00 \times 10^{-11}$ & 1 \\
\hline (R77) & $\mathrm{ALD}+\mathrm{HO} \rightarrow \mathrm{ACO}_{3}+\mathrm{H}_{2} \mathrm{O}$ & $5.55 \times 10^{-12}$ & -331 & $1.69 \times 10^{-11}$ & 7 \\
\hline (R78) & $\mathrm{KET}+\mathrm{HO} \rightarrow \mathrm{KETP}+\mathrm{H}_{2} \mathrm{O}$ & Table $2 \mathrm{c}$ & & $6.87 \times 10^{-13}$ & 7 \\
\hline (R79) & $\mathrm{HKET}+\mathrm{HO} \rightarrow \mathrm{HO}_{2}+\mathrm{MGLY}+\mathrm{H}_{2} \mathrm{O}$ & $3.00 \times 10^{-12}$ & & $3.00 \times 10^{-12}$ & 7 \\
\hline (R80) & $\mathrm{GLY}+\mathrm{HO} \rightarrow \mathrm{HO}_{2}+2 \mathrm{CO}+\mathrm{H}_{2} \mathrm{O}$ & $1.14 \times 10^{-11}$ & & $1.14 \times 10^{-11}$ & 12 \\
\hline (R81) & $\mathrm{MGLY}+\mathrm{HO} \rightarrow \mathrm{ACO}_{3}+\mathrm{CO}+\mathrm{H}_{2} \mathrm{O}$ & $1.72 \times 10^{-11}$ & & $1.72 \times 10^{-11}$ & 7 \\
\hline (R82) & $\begin{array}{c}\mathrm{MACR}+\mathrm{HO} \rightarrow 0.51 \mathrm{TCO}_{3}+0.41 \mathrm{HKET}+0.08 \mathrm{MGLY}+0.41 \mathrm{CO}+ \\
0.08 \mathrm{HCHO}+0.49 \mathrm{HO}_{2}+0.49 \mathrm{XO}_{2}\end{array}$ & $1.86 \times 10^{-11}$ & -175 & $3.35 \times 10^{-11}$ & 7,13 \\
\hline$(\mathrm{R} 83)$ & $\begin{array}{l}\mathrm{DCB}+\mathrm{HO} \rightarrow 0.50 \mathrm{TCO}_{3}+0.50 \mathrm{HO}_{2}+0.50 \mathrm{XO}_{2}+0.35 \mathrm{UDD}+0.15 \\
\mathrm{GLY}+0.15 \mathrm{MGLY}\end{array}$ & $2.80 \times 10^{-11}$ & -175 & $5.04 \times 10^{-11}$ & 14 \\
\hline (R84) & $\mathrm{UDD}+\mathrm{HO} \rightarrow 0.88 \mathrm{ALD}+0.12 \mathrm{KET}+\mathrm{HO}_{2}$ & $2.70 \times 10^{-10}$ & & $2.70 \times 10^{-10}$ & 15 \\
\hline (R85) & $\mathrm{OP} 1+\mathrm{HO} \rightarrow 0.65 \mathrm{MO}_{2}+0.35 \mathrm{HCHO}+0.35 \mathrm{HO}$ & $2.93 \times 10^{-12}$ & -190 & $5.54 \times 10^{-12}$ & 7 \\
\hline
\end{tabular}


Table 2b. (continued)

\begin{tabular}{|c|c|c|c|c|c|}
\hline $\begin{array}{l}\text { Reaction } \\
\text { No. }\end{array}$ & Reaction & $\begin{array}{c}A \\
\mathrm{~cm}^{3} \mathrm{~s}^{-1}\end{array}$ & $\begin{array}{c}E / R \\
\mathrm{~K}\end{array}$ & $k^{\mathrm{a}}$ & Note \\
\hline$(\mathrm{R} 86)$ & $\begin{array}{l}\mathrm{OP} 2+\mathrm{HO} \\
\quad 0.07 \mathrm{XO}_{2}\end{array}$ & $3.40 \times 10^{-12}$ & -190 & $6.43 \times 10^{-12}$ & 16 \\
\hline (R87) & $\mathrm{PAA}+\mathrm{HO} \rightarrow 0.35 \mathrm{HCHO}+0.65 \mathrm{ACO}_{3}+0.35 \mathrm{HO}_{2}+0.35 \mathrm{XO}_{2}$ & $2.93 \times 10^{-12}$ & -190 & $5.54 \times 10^{-12}$ & 17 \\
\hline (R88) & $\mathrm{PAN}+\mathrm{HO} \rightarrow \mathrm{HCHO}+\mathrm{XO}_{2}+\mathrm{H}_{2} \mathrm{O}+\mathrm{NO}_{3}$ & $4.00 \times 10^{-14}$ & & $4.00 \times 10^{-14}$ & 1,18 \\
\hline (R89) & $\begin{array}{l}\text { TPAN }+\mathrm{HO} \rightarrow 0.60 \mathrm{HKET}+0.40 \mathrm{HCHO}+0.40 \mathrm{HO}_{2}+\mathrm{XO}_{2}+ \\
0.40 \mathrm{PAN}+0.60 \mathrm{NO}_{3}\end{array}$ & $3.25 \times 10^{-13}$ & -500 & $1.74 \times 10^{-12}$ & 9,19 \\
\hline (R90) & $\mathrm{ONIT}+\mathrm{HO} \rightarrow \mathrm{HC} 3 \mathrm{P}+\mathrm{NO}_{2}+\mathrm{H}_{2} \mathrm{O}$ & $5.31 \times 10^{-12}$ & 260 & $2.22 \times 10^{-12}$ & 21 \\
\hline \multicolumn{6}{|c|}{$\mathrm{NO}_{3}+$ Organic Cormpounds } \\
\hline (R92) & $\mathrm{ALD}+\mathrm{NO}_{3} \rightarrow \mathrm{ACO}_{3}+\mathrm{HNO}_{3}$ & $1.40 \times 10^{-12}$ & 1900 & $2.38 \times 10^{-15}$ & 1,22 \\
\hline (R93) & $\mathrm{GLY}+\mathrm{NO}_{3} \rightarrow \mathrm{HNO}_{3}+\mathrm{HO}_{2}+2 \mathrm{CO}$ & $2.90 \times 10^{-12}$ & 1900 & $4.94 \times 10^{-15}$ & 22,23 \\
\hline (R94) & $\mathrm{MGLY}+\mathrm{NO}_{3} \rightarrow \mathrm{HNO}_{3}+\mathrm{ACO}_{3}+\mathrm{CO}$ & $1.40 \times 10^{-12}$ & 1900 & $2.38 \times 10^{-15}$ & 24 \\
\hline (R95) & $\mathrm{MACR}+\mathrm{NO}_{3} \rightarrow 0.20 \mathrm{TCO}_{3}+0.20 \mathrm{HNO}_{3}+0.80 \mathrm{OLNN}+0.80 \mathrm{CO}$ & $8.27 \times 10^{-15}$ & 150 & $5.00 \times 10^{-15}$ & 25 \\
\hline (R96) & $\begin{array}{c}\mathrm{DCB}+\mathrm{NO}_{3} \rightarrow 0.50 \mathrm{TCO}_{3}+0.50 \mathrm{HO}_{2}+0.50 \mathrm{XO}_{2}+0.25 \mathrm{GLY}+ \\
0.25 \mathrm{ALD}+0.03 \mathrm{KET}+0.25 \mathrm{MGLY}+0.5 \mathrm{HNO}_{3}+0.5 \mathrm{NO}_{2}\end{array}$ & $2.87 \times 10^{-13}$ & 1000 & $1.00 \times 10^{-14}$ & 14 \\
\hline (R97) & $\mathrm{CSL}+\mathrm{NO}_{3} \rightarrow \mathrm{HNO}_{3}+\mathrm{PHO}$ & $2.20 \times 10^{-11}$ & & $2.20 \times 10^{-11}$ & 26 \\
\hline (R98) & $\mathrm{ETE}+\mathrm{NO}_{3} \rightarrow 0.80 \mathrm{OLNN}+0.20$ OLND & Table $2 \mathrm{c}$ & & $2.05 \times 10^{-16}$ & 7 \\
\hline (R99) & $\mathrm{OLT}+\mathrm{NO}_{3} \rightarrow 0.43 \mathrm{OLNN}+0.57 \mathrm{OLND}$ & $1.79 \times 10^{-13}$ & 450 & $3.95 \times 10^{-14}$ & 8,27 \\
\hline (R100) & $\mathrm{OLI}+\mathrm{NO}_{3} \rightarrow 0.11$ OLNN +0.89 OLND & $8.64 \times 10^{-13}$ & -450 & $3.91 \times 10^{-12}$ & 8,27 \\
\hline (R101) & DIEN $+\mathrm{NO}_{3} \rightarrow 0.90$ OLNN +0.10 OLND $+0.90 \mathrm{MACR}$ & $1.0 \times 10^{-13}$ & & $1.0 \times 10^{-13}$ & 28 \\
\hline (R102) & $\mathrm{ISO}+\mathrm{NO}_{3} \rightarrow 0.90 \mathrm{OLNN}+0.10 \mathrm{OLND}+0.90 \mathrm{MACR}$ & $4.00 \times 10^{-12}$ & 446 & $8.96 \times 10^{-13}$ & 29 \\
\hline (R103) & $\mathrm{API}+\mathrm{NO}_{3} \rightarrow 0.10 \mathrm{OLNN}+0.90$ OLND & $1.19 \times 10^{-12}$ & -490 & $6.16 \times 10^{-12}$ & 7 \\
\hline (R104) & $\mathrm{LIM}+\mathrm{NO}_{3} \rightarrow 0.13 \mathrm{OLNN}+0.87$ OLND & $1.22 \times 10^{11}$ & & $1.22 \times 10^{-11}$ & 7 \\
\hline (R105) & $\begin{array}{l}\mathrm{TPAN}+\mathrm{NO}_{3} \rightarrow 0.60 \mathrm{ONIT}+0.60 \mathrm{NO}_{3}+0.40 \mathrm{PAN}+0.40 \mathrm{HCHO}+ \\
\quad 0.40 \mathrm{NO}_{2}+\mathrm{XO}_{2}\end{array}$ & $2.20 \times 10^{-14}$ & 500 & $4.11 \times 10^{-15}$ & $\begin{array}{r}9,19 \\
30\end{array}$ \\
\hline (R106) & $\begin{array}{l}O_{3}+\text { Organic Compounds } \\
\mathrm{ETE}+\mathrm{O}_{3} \rightarrow \mathrm{HCHO}+0.43 \mathrm{CO}+0.37 \mathrm{ORA} 1+0.26 \mathrm{HO}_{2}+0.13 \mathrm{H}_{2} \\
\quad+0.12 \mathrm{HO}\end{array}$ & $9.14 \times 10^{-15}$ & 2580 & $1.59 \times 10^{-18}$ & 7 \\
\hline (R107) & $\begin{array}{l}\mathrm{OLT}+\mathrm{O}_{3} \rightarrow 0.64 \mathrm{HCHO}+0.44 \mathrm{ALD}+0.37 \mathrm{CO}+0.14 \mathrm{ORA} 1+0.10 \\
\mathrm{ORA} 2+0.25 \mathrm{HO}_{2}+0.40 \mathrm{HO}+0.03 \mathrm{KET}+0.03 \mathrm{KETP}+0.06 \mathrm{CH}_{4} \\
+0.05 \mathrm{H}_{2}+0.006 \mathrm{H}_{2} \mathrm{O}_{2}+0.03 \mathrm{ETH}+0.19 \mathrm{MO}_{2}+0.10 \mathrm{ETHP}\end{array}$ & $4.33 \times 10^{-15}$ & 1800 & $1.03 \times 10^{-17}$ & 8,31 \\
\hline (R108) & $\begin{array}{l}\mathrm{OLI}+\mathrm{O}_{3} \rightarrow 0.02 \mathrm{HCHO}+0.99 \mathrm{ALD}+0.16 \mathrm{KET}+0.30 \mathrm{CO}+0.011 \\
\mathrm{H}_{2} \mathrm{O}_{2}+0.14 \mathrm{ORA} 2+0.07 \mathrm{CH}_{4}+0.22 \mathrm{HO}_{2}+0.63 \mathrm{HO}+0.23 \mathrm{MO}_{2} \\
+0.12 \mathrm{KETP}+0.06 \mathrm{ETH}+0.18 \mathrm{ETHP}\end{array}$ & $4.40 \times 10^{-15}$ & 845 & $2.58 \times 10^{-16}$ & 8,31 \\
\hline (R109) & $\begin{array}{c}\text { DIEN }+\mathrm{O}_{3} \rightarrow 0.90 \mathrm{HCHO}+0.39 \mathrm{MACR}+0.36 \mathrm{CO}+0.15 \mathrm{ORA} 1+ \\
0.09 \mathrm{O}^{3} P+0.30 \mathrm{HO}_{2}+0.35 \mathrm{OLT}+0.28 \mathrm{HO}+0.05 \mathrm{H}_{2}+0.15 \\
\mathrm{ACO}_{3}+0.03 \mathrm{MO}_{2}+0.02 \mathrm{KETP}+0.13 \mathrm{XO}_{2}+0.001 \mathrm{H}_{2} \mathrm{O}_{2}\end{array}$ & $1.34 \times 10^{-14}$ & 2283 & $6.33 \times 10^{-18}$ & 7 \\
\hline (R110) & $\begin{array}{c}\mathrm{ISO}+\mathrm{O}_{3} \rightarrow 0.90 \mathrm{HCHO}^{3}+0.39 \mathrm{MACR}+0.36 \mathrm{CO}+0.15 \mathrm{ORA} 1+ \\
0.09 \mathrm{O}^{3} \mathrm{P}+0.30 \mathrm{HO}_{2}+0.35 \mathrm{OLT}+0.28 \mathrm{HO}+0.05 \mathrm{H}_{2} \mid 0.15 \\
\mathrm{ACO}_{3}+0.03 \mathrm{MO}_{2}+0.02 \mathrm{KETP}+0.13 \mathrm{XO}_{2}+0.001 \mathrm{H}_{2} \mathrm{O}_{2}\end{array}$ & $7.86 \times 10^{-15}$ & 1913 & $1.28 \times 10^{-17}$ & 7 \\
\hline (R111) & $\begin{array}{l}\mathrm{API}+\mathrm{O}_{3} \rightarrow 0.65 \mathrm{ALD}+0.53 \mathrm{KET}+0.14 \mathrm{CO}+0.20 \mathrm{ETHP}+0.42 \\
\mathrm{KETP}+0.85 \mathrm{HO}+0.10 \mathrm{HO} 2+0.02 \mathrm{H}_{2} \mathrm{O}_{2}\end{array}$ & $1.01 \times 10^{-15}$ & 736 & $8.66 \times 10^{-17}$ & 7 \\
\hline (R112) & $\begin{array}{l}\mathrm{LIM}+\mathrm{O}_{3} \rightarrow 0.04 \mathrm{HCHO}+0.46 \mathrm{OLT}+0.14 \mathrm{CO}+0.16 \mathrm{ETHP}+0.42 \\
\mathrm{KETP}+0.85 \mathrm{HO}+0.10 \mathrm{HO}_{2}+0.02 \mathrm{H}_{2} \mathrm{O}_{2}+0.79 \mathrm{MACR}+0.01 \\
\text { ORA } 1+0.07 \text { ORA2 }\end{array}$ & $2.00 \times 10^{-16}$ & & $2.00 \times 10^{-16}$ & 7 \\
\hline (R113) & $\begin{array}{l}\text { MACR }+\mathrm{O}_{3} \rightarrow 0.40 \mathrm{HCHO}+0.60 \mathrm{MGLY}+0.13 \mathrm{ORA} 2+0.54 \mathrm{CO}+ \\
0.08 \mathrm{H}_{2}+0.22 \mathrm{ORA} 1+0.29 \mathrm{HO}_{2}+0.07 \mathrm{HO}+0.13 \mathrm{OP} 2+0.13 \mathrm{ACO}_{3}\end{array}$ & $1.36 \times 10^{-15}$ & 2112 & $1.14 \times 10^{-18}$ & 32 \\
\hline (R114) & $\begin{array}{l}\mathrm{DCB}+\mathrm{O}_{3} \rightarrow 0.21 \mathrm{HO}+0.29 \mathrm{HO}_{2}+0.66 \mathrm{CO}+0.50 \mathrm{GLY}+0.28 \\
\mathrm{ACO}_{3}+0.16 \mathrm{ALD}+0.62 \mathrm{MGLY}+0.11 \mathrm{PAA}+0.11 \mathrm{ORA} 1+0.21 \\
\mathrm{ORA}_{2}\end{array}$ & $2.00 \times 10^{-18}$ & & $2.00 \times 10^{-18}$ & 14 \\
\hline (R115) & $\begin{array}{l}\mathrm{TPAN}+\mathrm{O}_{3} \rightarrow 0.70 \mathrm{HCHO}+0.30 \mathrm{PAN}+0.70 \mathrm{NO}_{2}+0.13 \mathrm{CO}+0.04 \\
\mathrm{H}_{2}+0.11 \text { ORA } 1+0.08 \mathrm{HO}_{2}+0.036 \mathrm{HO}+0.70 \mathrm{ACO}_{3}\end{array}$ & $2.46 \times 10^{-15}$ & 1700 & $8.19 \times 10^{-18}$ & 9,19 \\
\hline \multicolumn{6}{|c|}{ Reactions of Intermediates Produced by Aromatic Oxidation } \\
\hline (R116) & $\mathrm{PHO}+\mathrm{NO}_{2} \rightarrow 0.10 \mathrm{CSL}+\mathrm{ONIT}$ & $2.00 \times 10^{-11}$ & & $2.00 \times 10^{-11}$ & 34 \\
\hline & $\mathrm{PHO}+\mathrm{HO}_{2} \rightarrow \mathrm{CSL}$ & $1.00 \times 10^{-11}$ & & $1.00 \times 10^{-11}$ & 35 \\
\hline (R118) & $\mathrm{ADDT}+\mathrm{NO}_{2} \rightarrow \mathrm{CSL}+\mathrm{IONO}$ & $3.60 \times 10^{-11}$ & & $3.60 \times 10^{-11}$ & 7 \\
\hline (R119) & $\mathrm{ADDT}+\mathrm{O}_{2} \rightarrow 0.98 \mathrm{TOLP}+0.02 \mathrm{CSL}+0.02 \mathrm{HO}_{2}$ & $1.66 \times 10^{-17}$ & -1044 & $5.52 \times 10^{-16}$ & 7 \\
\hline (R120) & $\mathrm{ADDT}+\mathrm{O}_{3} \rightarrow \mathrm{CSL}+\mathrm{HO}$ & $5.00 \times 10^{-11}$ & & $5.00 \times 10^{-11}$ & 36 \\
\hline 121) & $\mathrm{ADDX}+\mathrm{NO}_{2} \rightarrow \mathrm{CSL}+\mathrm{HONO}$ & $3.60 \times 10^{-11}$ & & $3.60 \times 10^{-11}$ & 37 \\
\hline (R122) & $\mathrm{ADDX}+\mathrm{O}_{2} \rightarrow 0.98 \mathrm{XYLP}+0.02 \mathrm{CSL}+0.02 \mathrm{HO}_{2}$ & $1.66 \times 10^{-17}$ & -1044 & $5.52 \times 10^{-16}$ & 37 \\
\hline (R123) & $\mathrm{ADDX}+\mathrm{O}_{3} \rightarrow \mathrm{CSL}+\mathrm{HO}$ & $1.00 \times 10^{-11}$ & & $1.00 \times 10^{-11}$ & 37 \\
\hline (R124) & $\mathrm{ADDC}+\mathrm{NO}_{2} \rightarrow \mathrm{CSL}+\mathrm{HONO}$ & $3.60 \times 10^{-11}$ & & $3.60 \times 10^{-11}$ & 37 \\
\hline (R125) & $\mathrm{ADDC}+\mathrm{O}_{2} \rightarrow 0.98 \mathrm{CSLP}+0.02 \mathrm{CSL}+0.02 \mathrm{HO}_{2}$ & $1.66 \times 10^{-17}$ & -1044 & $5.52 \times 10^{-16}$ & 37 \\
\hline (R126) & $\mathrm{ADDC}+\mathrm{O}_{3} \rightarrow \mathrm{CSL}+\mathrm{HO}$ & $5.00 \times 10^{11}$ & & $5.00 \times 10^{-11}$ & 37 \\
\hline \multicolumn{6}{|c|}{ Peroxyacylnitrate Formation and Decomposition } \\
\hline (R127) & $\mathrm{ACO}_{3}+\mathrm{NO}_{2} \rightarrow \mathrm{PAN}$ & & & $8.66 \times 10^{-12}$ & \\
\hline & $\mathrm{PAN} \rightarrow \mathrm{ACO}_{3}+\mathrm{NO}_{2}$ & Table $2 \mathrm{e}$ & & $4.63 \times 10^{-4}$ & 38 \\
\hline (R129) & $\mathrm{TCO}_{3}+\mathrm{NO}_{2} \rightarrow$ TPAN & Table $2 \mathrm{~d}$ & & $8.66 \times 10^{-12}$ & 38 \\
\hline (R130) & $\mathrm{TPAN} \rightarrow \mathrm{TCO}_{3}+\mathrm{NO}_{2}$ & Table $2 \mathrm{e}$ & & $4.63 \times 10^{4}$ & 38 \\
\hline
\end{tabular}


Table 2b. (continued)

\begin{tabular}{|c|c|c|c|c|c|}
\hline $\begin{array}{l}\text { Reaction } \\
\quad \text { No. }\end{array}$ & Reaction & $\begin{array}{c}A \\
\mathrm{~cm}^{3} \mathrm{~s}^{-1}\end{array}$ & $\begin{array}{l}E / R \\
\mathrm{~K}\end{array}$ & $k^{\mathrm{a}}$ & Note \\
\hline \multicolumn{6}{|c|}{$N O+$ Organic Peroxy Radicals } \\
\hline (R131) & $\mathrm{MO}_{2}+\mathrm{NO} \rightarrow \mathrm{HCHO}+\mathrm{HO}_{2}+\mathrm{NO}_{2}$ & $4.2 \times 10^{-12}$ & -180 & $7.68 \times 10^{-12}$ & 1 \\
\hline (R132) & $\mathrm{ETHP}+\mathrm{NO} \rightarrow \mathrm{ALD}+\mathrm{HO}_{2}+\mathrm{NO}_{2}$ & $8.7 \times 10^{-12}$ & & $8.70 \times 10^{-12}$ & 1 \\
\hline (R133) & $\begin{array}{l}\mathrm{HC} 3 \mathrm{P}+\mathrm{NO} \rightarrow 0.047 \mathrm{HCHO}+0.233 \mathrm{ALD}+0.623 \mathrm{KET}+0.063 \mathrm{GLY} \\
\quad+0.742 \mathrm{HO}_{2}+0.15 \mathrm{MO}_{2}+0.048 \mathrm{ETHP}+0.048 \mathrm{XO}_{2}+0.059 \mathrm{ONIT} \\
\quad+0.941 \mathrm{NO}_{2}\end{array}$ & $4.0 \times 10^{-12}$ & & $4.00 \times 10^{-12}$ & 38 \\
\hline (R134) & $\begin{array}{l}\mathrm{HC} 5 \mathrm{P}+\mathrm{NO} \rightarrow 0.021 \mathrm{HCHO}+0.211 \mathrm{ALD}+0.722 \mathrm{KET}+0.599 \mathrm{HO}_{2} \\
\quad+0.031 \mathrm{MO}_{2}+0.245 \mathrm{ETHP}+0.334 \mathrm{XO}_{2}+0.124 \mathrm{ONIT}+0.876 \mathrm{NO}_{2}\end{array}$ & $4.0 \times 10^{-12}$ & & $4.00 \times 10^{-12}$ & 38 \\
\hline (R135) & $\begin{array}{l}\mathrm{HC} 8 \mathrm{P}+\mathrm{NO} \rightarrow 0.15 \mathrm{ALD}+0.642 \mathrm{KET}+0.133 \mathrm{ETHP}+0.261 \mathrm{ONIT} \\
\quad+0.739 \mathrm{NO}_{2}+0.606 \mathrm{HO}_{2}+0.416 \mathrm{XO}_{2}\end{array}$ & $4.0 \times 10^{-12}$ & & $4.00 \times 10^{-12}$ & 38 \\
\hline (R136) & $\mathrm{ETEP}+\mathrm{NO} \rightarrow 1.6 \mathrm{HCHO}+\mathrm{HO}_{2}+\mathrm{NO}_{2}+0.2 \mathrm{ALD}$ & $9.0 \times 10^{-12}$ & & $9.00 \times 10^{-12}$ & 7,39 \\
\hline (R137) & $\mathrm{OLTP}+\mathrm{NO} \rightarrow 0.94 \mathrm{ALD}+\dot{\mathrm{HCHO}}+\mathrm{HO}_{2}+\mathrm{NO}_{2}+0.06 \mathrm{KET}$ & $4.0 \times 10^{-12}$ & & $4.00 \times 10^{-12}$ & 38 \\
\hline (R138) & $\mathrm{OLIP}+\mathrm{NO} \rightarrow \mathrm{HO}_{2}+1.71 \mathrm{ALD}+0.29 \mathrm{KET}+\mathrm{NO}_{2}$ & $4.0 \times 10^{-12}$ & & $4.00 \times 10^{-12}$ & 38 \\
\hline (R139) & $\begin{array}{l}\text { ISOP + NO } \rightarrow 0.446 \mathrm{MACR}+0.354 \mathrm{OLT}+0.847 \mathrm{HO}_{2}+0.606 \\
\mathrm{HCHO}+0.153 \mathrm{ONIT}+0.847 \mathrm{NO}_{2}\end{array}$ & $4.0 \times 10^{-12}$ & & $4.00 \times 10^{-12}$ & 38 \\
\hline (R140) & $\begin{array}{l}\mathrm{APIP}+\mathrm{NO} \rightarrow 0.80 \mathrm{HO}_{2}+0.80 \mathrm{ALD}+0.80 \mathrm{KET}+0.20 \mathrm{ONIT}+ \\
\quad 0.80 \mathrm{NO}_{2}\end{array}$ & $4.0 \times 10^{-12}$ & & $4.0 \times 10^{-12}$ & 38 \\
\hline (R141) & $\begin{array}{c}\text { LIMP }+\mathrm{NO} \rightarrow 0.65 \mathrm{HO}_{2}+0.40 \mathrm{MACR}+0.25 \mathrm{OLI}+0.25 \mathrm{HCHO}+ \\
0.35 \mathrm{ONIT}+0.65 \mathrm{NO}_{2}\end{array}$ & $4.0 \times 10^{-12}$ & & $4.0 \times 10^{-12}$ & 38 \\
\hline (R142) & $\begin{array}{c}\mathrm{TOLP}+\mathrm{NO} \rightarrow 0.95 \mathrm{NO}_{2}+0.95 \mathrm{HO}_{2}+0.65 \mathrm{MGLY}+1.20 \mathrm{GLY}+ \\
0.50 \mathrm{DCB}+0.05 \mathrm{ONIT}\end{array}$ & $4.0 \times 10^{-12}$ & & $4.00 \times 10^{-12}$ & 38 \\
\hline (R143) & $\begin{array}{l}\mathrm{XYLP}+\mathrm{NO} \rightarrow 0.95 \mathrm{NO}_{2}+0.95 \mathrm{HO}_{2}+0.60 \mathrm{MGLY}+0.35 \mathrm{GLY}+ \\
0.95 \mathrm{DCB}+0.05 \mathrm{ONIT}\end{array}$ & $4.0 \times 10^{-12}$ & & $4.00 \times 10^{-12}$ & 38 \\
\hline (R144) & $\mathrm{CSLP}+\mathrm{NO} \rightarrow \mathrm{GLY}+\mathrm{MGLY}+\mathrm{HO}_{2}+\mathrm{NO}_{2}$ & $4.0 \times 10^{-12}$ & & $4.00 \times 10^{-12}$ & 38 \\
\hline (R145) & $\mathrm{ACO}_{3}+\mathrm{NO} \rightarrow \mathrm{MO}_{2}+\mathrm{NO}_{2}$ & $2.0 \times 10^{-11}$ & & $2.00 \times 10^{-11}$ & 7,39 \\
\hline (R146) & $\mathrm{TCO}_{3}+\mathrm{NO} \rightarrow \mathrm{ACO}_{3}+\mathrm{HCHO}+\mathrm{NO}_{2}$ & $2.0 \times 10^{-11}$ & & $2.00 \times 10^{-11}$ & 38 \\
\hline (R147) & $\begin{array}{l}\mathrm{KETP}+\mathrm{NO} \rightarrow 0.54 \mathrm{MGLY}+0.46 \mathrm{ALD}+0.23 \mathrm{ACO}_{3}+0.77 \mathrm{HO}_{2}+ \\
0.16 \mathrm{XO}_{2}+\mathrm{NO}_{2}\end{array}$ & $4.0 \times 10^{-12}$ & & $4.00 \times 10^{-12}$ & 38 \\
\hline (R148) & $\mathrm{OLNN}+\mathrm{NO} \rightarrow \mathrm{HO}_{2}+\mathrm{ONIT}+\mathrm{NO}_{2}$ & $4.0 \times 10^{-12}$ & & $4.00 \times 10^{-12}$ & 38 \\
\hline (R149) & $\mathrm{OLND}+\mathrm{NO} \rightarrow 0.287 \mathrm{HCHO}+1.24 \mathrm{ALD}+0.464 \mathrm{KET}+2 \mathrm{NO}_{2}$ & $4.0 \times 10^{-12}$ & & $4.00 \times 10^{-12}$ & 38 \\
\hline \multicolumn{6}{|c|}{$\mathrm{HO}_{2}+$ Organic Peroxy Radicals } \\
\hline$(\mathrm{R} 150)$ & $\mathrm{MO}_{2}+\mathrm{HO}_{2} \rightarrow \mathrm{OP} 1$ & $3.8 \times 10^{-13}$ & -800 & $5.57 \times 10^{-12}$ & 1 \\
\hline (R151) & $\mathrm{ETHP}+\mathrm{HO}_{2} \rightarrow \mathrm{OP} 2$ & $7.5 \times 10^{-13}$ & -700 & $7.86 \times 10^{-12}$ & 1 \\
\hline (R152) & $\mathrm{HC} 3 \mathrm{P}+\mathrm{HO}_{2} \rightarrow \mathrm{OP} 2$ & $1.66 \times 10^{-13}$ & -1300 & $1.30 \times 10^{-11}$ & 38 \\
\hline (R153) & $\mathrm{HC} 5 \mathrm{P}+\mathrm{HO}_{2} \rightarrow \mathrm{OP} 2$ & $1.66 \times 10^{-13}$ & -1300 & $1.30 \times 10^{-11}$ & 38 \\
\hline (R154) & $\mathrm{HC} 8 \mathrm{P}+\mathrm{HO}_{2} \rightarrow \mathrm{OP} 2$ & $1.66 \times 10^{-13}$ & -1300 & $1.30 \times 10^{-11}$ & 38 \\
\hline (R155) & $\mathrm{ETEP}+\mathrm{HO}_{2} \rightarrow \mathrm{OP} 2$ & $1.9 \times 10^{-13}$ & -1300 & $1.50 \times 10^{-11}$ & 38,40 \\
\hline (R156) & $\mathrm{OLTP}+\mathrm{HO}_{2} \rightarrow \mathrm{OP} 2$ & $1.66 \times 10^{-13}$ & -1300 & $1.30 \times 10^{-11}$ & 38 \\
\hline (R157) & $\mathrm{OLIP}+\mathrm{HO}_{2} \rightarrow \mathrm{OP} 2$ & $1.66 \times 10^{-13}$ & -1300 & $1.30 \times 10^{-11}$ & 38 \\
\hline (R158) & $\mathrm{ISOP}+\mathrm{HO}_{2} \rightarrow \mathrm{OP} 2$ & $1.28 \times 10^{-13}$ & -1300 & $1.00 \times 10^{11}$ & 41 \\
\hline (R159) & $\mathrm{APIP}+\mathrm{HO}_{2} \rightarrow \mathrm{OP} 2$ & $1.50 \times 10^{-11}$ & & $1.50 \times 10^{-11}$ & 9 \\
\hline (R160) & $\mathrm{LIMP}+\mathrm{HO}_{2} \rightarrow \mathrm{OP} 2$ & $1.50 \times 10^{-11}$ & & $1.50 \times 10^{-11}$ & 9 \\
\hline (R161) & $\mathrm{TOLP}+\mathrm{HO}_{2} \rightarrow \mathrm{OP} 2$ & $3.75 \times 10^{-13}$ & -980 & $1.01 \times 10^{-11}$ & 38,40 \\
\hline (R162) & $\mathrm{XYLP}+\mathrm{HO}_{2} \rightarrow \mathrm{OP} 2$ & $3.75 \times 10^{-13}$ & -980 & $1.01 \times 10^{-11}$ & 38,40 \\
\hline (R163) & $\mathrm{CSLP}+\mathrm{HO}_{2} \rightarrow \mathrm{OP} 2$ & $3.75 \times 10^{-13}$ & -980 & $1.01 \times 10^{-11}$ & 38,40 \\
\hline (R164) & $\mathrm{ACO}_{3}+\mathrm{HO}_{2} \rightarrow \mathrm{PAA}$ & $1.15 \times 10^{-12}$ & -550 & $7.28 \times 10^{-12}$ & 40 \\
\hline (R165) & $\mathrm{ACO}_{3}+\mathrm{HO}_{2} \rightarrow \mathrm{ORA} 2+\mathrm{O}_{3}$ & $3.86 \times 10^{-16}$ & -2640 & $2.72 \times 10^{-12}$ & 40 \\
\hline (R166) & $\mathrm{TCO}_{3}+\mathrm{HO}_{2} \rightarrow \mathrm{OP} 2$ & $1.15 \times 10^{-12}$ & -550 & $7.28 \times 10^{-12}$ & 38 \\
\hline (R167) & $\mathrm{TCO}_{3}+\mathrm{HO}_{2} \rightarrow \mathrm{ORA} 2+\mathrm{O}_{3}$ & $3.86 \times 10^{-16}$ & -2640 & $2.72 \times 10^{-12}$ & 38 \\
\hline (R168) & $\mathrm{KETP}+\mathrm{HO}_{2} \rightarrow \mathrm{OP} 2$ & $1.15 \times 10^{-13}$ & -1300 & $9.02 \times 10^{-12}$ & 38,40 \\
\hline (R169) & $\mathrm{OLNN}+\mathrm{HO}_{2} \rightarrow \mathrm{ONIT}$ & $1.66 \times 10^{-13}$ & -1300 & $1.30 \times 10^{-11}$ & 38 \\
\hline (R170) & $\mathrm{OLND}+\mathrm{HO}_{2} \rightarrow$ ONIT & $1.66 \times 10^{-13}$ & -1300 & $1.30 \times 10^{11}$ & 38 \\
\hline \multicolumn{6}{|c|}{ Methyl Peroxy Radical + Organic Peroxy Radicals } \\
\hline$(\mathrm{R} 171)$ & $\mathrm{MO}_{2}+\mathrm{MO}_{2} \rightarrow 1.33 \mathrm{HCHO}+0.66 \mathrm{HO}_{2}$ & $9.1 \times 10^{-14}$ & -416 & $3.68 \times 10^{-13}$ & 39 \\
\hline (R172) & $\mathrm{ETHP}+\mathrm{MO}_{2} \rightarrow 0.75 \mathrm{HCHO}+\mathrm{HO}_{2}+0.75 \mathrm{ALD}$ & $1.18 \times 10^{-13}$ & -158 & $2.01 \times 10^{-13}$ & 38,40 \\
\hline (R173) & $\begin{array}{l}\mathrm{HC} 3 \mathrm{P}+\mathrm{MO}_{2} \rightarrow 0.81 \mathrm{HCHO}+0.992 \mathrm{HO}_{2}+0.58 \mathrm{ALD}+0.018 \mathrm{KET} \\
\quad+0.007 \mathrm{MO}_{2}+0.005 \mathrm{MGLY}+0.085 \mathrm{XO}_{2}+0.119 \mathrm{GLY}\end{array}$ & $9.46 \times 10^{-14}$ & -431 & $4.02 \times 10^{-13}$ & 38 \\
\hline (R174) & $\begin{array}{l}\mathrm{HC} 5 \mathrm{P}+\mathrm{MO}_{2} \rightarrow 0.829 \mathrm{HCHO}+0.946 \mathrm{HO}_{2}+0.523 \mathrm{ALD}+0.24 \mathrm{KET} \\
\quad+0.014 \mathrm{ETHP}+0.049 \mathrm{MO}_{2}+0.245 \mathrm{XO}_{2}\end{array}$ & $1.00 \times 10^{-13}$ & -467 & $4.79 \times 10^{-13}$ & 38 \\
\hline (R175) & $\begin{array}{l}\mathrm{HC} 8 \mathrm{P}+\mathrm{MO}_{2} \rightarrow 0.753 \mathrm{HCHO}+0.993 \mathrm{HO}_{2}+0.411 \mathrm{ALD}+0.419 \mathrm{KET} \\
\quad+0.322 \mathrm{XO}_{2}+0.013 \mathrm{ETHP}\end{array}$ & $4.34 \times 10^{-14}$ & -633 & $3.63 \times 10^{-13}$ & 38 \\
\hline (R176) & $\mathrm{ETEP}+\mathrm{MO}_{2} \rightarrow 1.55 \mathrm{HCHO}+\mathrm{HO}_{2}+0.35 \mathrm{ALD}$ & $1.71 \times 10^{-13}$ & -708 & $1.84 \times 10^{-12}$ & 38 \\
\hline (R177) & $\mathrm{OLTP}+\mathrm{MO}_{2} \rightarrow 1.25 \mathrm{HCHO}+\mathrm{HO}_{2}+0.669 \mathrm{ALD}+0.081 \mathrm{KET}$ & $1.46 \times 10^{-13}$ & -708 & $1.57 \times 10^{-12}$ & 38 \\
\hline (R178) & OLIP $+\mathrm{MO}_{2} \rightarrow 0.755 \mathrm{HCHO}+\mathrm{HO}_{2}+0.932 \mathrm{ALD}+0.313 \mathrm{KET}$ & $9.18 \times 10^{-14}$ & -708 & $9.87 \times 10^{-13}$ & 38 \\
\hline (R179) & $\begin{array}{l}\mathrm{ISOP}+\mathrm{MO}_{2} \\
1.09 \mathrm{HCHO}\end{array}$ & $1.36 \times 10^{-13}$ & -708 & $1.46 \times 10^{-12}$ & 9 \\
\hline (R180) & $\mathrm{APIP}+\mathrm{MO}_{2} \rightarrow \mathrm{HCHO}+\mathrm{ALD}+\mathrm{KET}+2 \mathrm{HO}_{2}$ & $3.56 \times 10^{-14}$ & -708 & $3.83 \times 10^{-13}$ & 9 \\
\hline (R181) & $\mathrm{LIMP}+\mathrm{MO}_{2} \rightarrow 1.4 \mathrm{HCHO}+0.60 \mathrm{MACR}+0.40 \mathrm{OLI}+2 \mathrm{HO}_{2}$ & $3.56 \times 10^{-14}$ & -708 & $3.83 \times 10^{-13}$ & 9 \\
\hline (R182) & $\mathrm{TOLP}+\mathrm{MO}_{2} \rightarrow \mathrm{IICHO}+\mathrm{HO}_{2}+0.35 \mathrm{MGLY}+0.65 \mathrm{GLY}+\mathrm{DCB}$ & $3.56 \times 10^{-14}$ & -708 & $3.83 \times 10^{-13}$ & 38 \\
\hline (R183) & $\mathrm{XYLP}+\mathrm{MO}_{2} \rightarrow \mathrm{HCHO}+\mathrm{HO}_{2}+0.63 \mathrm{MGLY}+0.37 \mathrm{GLY}+\mathrm{DCB}$ & $3.56 \times 10^{-14}$ & -708 & $3.83 \times 10^{-13}$ & 38 \\
\hline (R184) & $\mathrm{CSLP}+\mathrm{MO}_{2} \rightarrow \mathrm{GLY}+\mathrm{MGLY}+\mathrm{HCHO}+2 \mathrm{HO}_{2}$ & $3.56 \times 10^{-14}$ & -708 & $3.83 \times 10^{-13}$ & 38 \\
\hline
\end{tabular}


Table 2b. (continued)

\begin{tabular}{|c|c|c|c|c|c|}
\hline $\begin{array}{l}\text { Reaction } \\
\text { No. }\end{array}$ & Reaction & $\begin{array}{c}A, \\
\mathrm{~cm}^{3} \mathrm{~s}^{-1}\end{array}$ & $E / R$ & $k^{\mathrm{a}}$ & Note \\
\hline (R185) & $\mathrm{ACO}_{3}+\mathrm{MO}_{2} \rightarrow \mathrm{HCHO}+\mathrm{HO}_{2}+\mathrm{MO}_{2}$ & $3.21 \times 10^{-11}$ & 440 & $7.33 \times 10^{-12}$ & 38 \\
\hline (R186) & $\mathrm{ACO}_{3}+\mathrm{MO}_{2} \rightarrow \mathrm{HCHO}+$ ORA2 & $2.68 \times 10^{-16}$ & -2510 & $1.22 \times 10^{-12}$ & 38 \\
\hline (R187) & $\mathrm{TCO}_{3}+\mathrm{MO}_{2} \rightarrow 2 \mathrm{HCHO}+\mathrm{HO}_{2}+\mathrm{ACO}_{3}$ & $3.21 \times 10^{-11}$ & 440 & $7.33 \times 10^{-12}$ & 38 \\
\hline (R188) & $\mathrm{TCO}_{3}+\mathrm{MO}_{2} \rightarrow \mathrm{HCHO}+\mathrm{ORA} 2$ & $2.68 \times 10^{-16}$ & -2510 & $1.22 \times 10^{-12}$ & 38 \\
\hline (R189) & $\begin{array}{l}\text { KETP }+\mathrm{MO}_{2} \rightarrow 0.75 \mathrm{HCHO}+0.88 \mathrm{HO}_{2}+0.40 \mathrm{MGLY}+0.30 \mathrm{ALD} \\
\quad+0.30 \mathrm{HKET}+0.12 \mathrm{ACO}_{3}+0.08 \mathrm{XO}_{2}\end{array}$ & $6.91 \times 10^{-13}$ & -508 & $3.80 \times 10^{-12}$ & 38 \\
\hline (R190) & $\mathrm{OLNN}+\mathrm{MO}_{2} \rightarrow 0.75 \mathrm{HCHO}+\mathrm{HO}_{2}+$ ONIT & $1.60 \times 10^{-13}$ & -708 & $1.72 \times 10^{-12}$ & 38 \\
\hline (R191) & $\begin{array}{l}\text { OLND }+\mathrm{MO}_{2} \rightarrow 0.96 \mathrm{HCHO}+0.5 \mathrm{HO}_{2}+0.64 \mathrm{ALD}+0.149 \mathrm{KET}+ \\
0.5 \mathrm{NO}_{2}+0.5 \text { ONIT }\end{array}$ & $9.68 \times 10^{-14}$ & -708 & $1.04 \times 10^{-12}$ & 38 \\
\hline \multicolumn{6}{|c|}{ Acetyl Radical + Organic Peroxy Radicals } \\
\hline (R192) & $\mathrm{ETHP}+\mathrm{ACO}_{3} \rightarrow \mathrm{ALD}+0.5 \mathrm{HO}_{2}+0.5 \mathrm{MO}_{2}+0.5 \mathrm{ORA} 2$ & $1.03 \times 10^{-12}$ & -211 & $2.09 \times 10^{-12}$ & 38 \\
\hline (R193) & $\begin{array}{l}\mathrm{HC} 3 \mathrm{P}+\mathrm{ACO}_{3} \rightarrow 0.724 \mathrm{ALD}+0.127 \mathrm{KET}+0.488 \mathrm{HO}_{2}+0.508 \mathrm{MO}_{2} \\
\quad+0.006 \mathrm{ETHP}+0.071 \mathrm{XO}_{2}+0.091 \mathrm{HCHO}+0.10 \mathrm{GLY}+0.499 \\
\mathrm{ORA} 2+0.004 \mathrm{MGLY}\end{array}$ & $6.90 \times 10^{-13}$ & -460 & $3.23 \times 10^{-12}$ & 38 \\
\hline (R194) & $\begin{array}{l}\mathrm{HC} 5 \mathrm{P}+\mathrm{ACO}_{3} \rightarrow 0.677 \mathrm{ALD}+0.33 \mathrm{KET}+0.438 \mathrm{HO}_{2}+0.554 \mathrm{MO}_{2} \\
\quad+0.495 \mathrm{ORA} 2+0.018 \mathrm{ETHP}+0.237 \mathrm{XO}_{2}+0.076 \mathrm{HCHO}\end{array}$ & $5.59 \times 10^{-13}$ & -522 & $3.22 \times 10^{-12}$ & 38 \\
\hline (R195) & $\begin{array}{l}\mathrm{HC} 8 \mathrm{P}+\mathrm{ACO}_{3} \rightarrow 0.497 \mathrm{ALD}+0.581 \mathrm{KET}+0.489 \mathrm{HO}_{2}+0.507 \mathrm{MO}_{2} \\
\quad+0.495 \mathrm{ORA} 2+0.015 \mathrm{ETHP}+0.318 \mathrm{XO}_{2}\end{array}$ & $2.47 \times 10^{-13}$ & -683 & $2.44 \times 10^{-12}$ & 38 \\
\hline (R196) & $\begin{array}{l}\mathrm{ETEP}+\mathrm{ACO}_{3} \rightarrow 0.8 \mathrm{HCHO}+0.6 \mathrm{ALD}+0.5 \mathrm{HO}_{2}+0.5 \mathrm{MO}_{2}+ \\
0.5 \mathrm{ORA} 2\end{array}$ & $9.48 \times 10^{-13}$ & -765 & $1.24 \times 10^{-11}$ & 38 \\
\hline (R197) & $\begin{array}{l}\text { OLTP }+\mathrm{ACO}_{3} \rightarrow 0.859 \text { ALD }+0.501 \mathrm{HCHO}+0.501 \mathrm{HO}_{2}+0.501 \\
\mathrm{MO}_{2}+0.499 \text { ORA } 2+0.141 \mathrm{KET}\end{array}$ & $8.11 \times 10^{-13}$ & -765 & $1.06 \times 10^{-11}$ & 38 \\
\hline (R198) & $\underset{0.49 \mathrm{ORA} 2}{\mathrm{OLIP}}+\mathrm{ACO}_{3} \rightarrow 0.941 \mathrm{ALD}+0.569 \mathrm{KET}+0.51 \mathrm{HO}_{2}+0.51 \mathrm{MO}_{2}+$ & $5.09 \times 10^{-13}$ & -765 & $6.63 \times 10^{-12}$ & 38 \\
\hline (R199) & $\begin{array}{l}\text { ISOP }+\mathrm{ACO}_{3} \rightarrow 0.771 \mathrm{MACR}+0.229 \mathrm{OLT}+0.506 \mathrm{HO}_{2}+0.494 \\
\text { ORA2 }+0.340 \mathrm{HCHO}+0.506 \mathrm{MO}_{2}\end{array}$ & $7.60 \times 10^{-13}$ & -765 & $9.90 \times 10^{-12}$ & 9 \\
\hline$(\mathrm{R} 200)$ & $\mathrm{APIP}+\mathrm{ACO}_{3} \rightarrow \mathrm{ALD}+\mathrm{KET}+\mathrm{HO}_{2}+\mathrm{MO}_{2}$ & $7.40 \times 10^{-13}$ & -765 & $9.63 \times 10^{-12}$ & 9 \\
\hline (R201) & $\underset{\mathrm{MO}_{2}}{\mathrm{LIMP}}+\mathrm{ACO}_{3} \rightarrow 0.60 \mathrm{MACR}+0.40 \mathrm{OLI}+0.40 \mathrm{HCHO}+\mathrm{HO}_{2}+$ & $7.40 \times 10^{-13}$ & -765 & $9.63 \times 10^{-12}$ & 9 \\
\hline (R202) & $\mathrm{TOLP}+\mathrm{ACO}_{3} \rightarrow \mathrm{MO}_{2}+\mathrm{HO}_{2}+0.35 \mathrm{MGLY}+0.65 \mathrm{GLY}+\mathrm{DCB}$ & $7.40 \times 10^{13}$ & -765 & $9.63 \times 10^{12}$ & 38 \\
\hline (R203) & $\mathrm{XYLP}+\mathrm{ACO}_{3} \rightarrow \mathrm{MO}_{2}+\mathrm{HO}_{2}+0.63 \mathrm{MGLY}+0.37 \mathrm{GLY}+\mathrm{DCB}$ & $7.40 \times 10^{-13}$ & -765 & $9.63 \times 10^{-12}$ & 38 \\
\hline (R204) & $\mathrm{CSLP}+\mathrm{ACO}_{3} \rightarrow \mathrm{GLY}+\mathrm{MGLY}+\mathrm{MO}_{2}+\mathrm{HO}_{2}$ & $7.40 \times 10^{-13}$ & -765 & $9.63 \times 10^{-12}$ & 38 \\
\hline (R205) & $\mathrm{ACO}_{3}+\mathrm{ACO}_{3} \rightarrow 2 \mathrm{MO}_{2}$ & $2.80 \times 10^{-12}$ & -530 & $1.66 \times 10^{-11}$ & 39 \\
\hline (R206) & $\mathrm{TCO}_{3}+\mathrm{ACO}_{3} \rightarrow \mathrm{MO}_{2}+\mathrm{ACO}_{3}+\mathrm{HCHO}$ & $2.80 \times 10^{-12}$ & -530 & $1.66 \times 10^{-11}$ & 38 \\
\hline (R207) & $\begin{array}{l}\mathrm{KETP}+\mathrm{ACO}_{3} \rightarrow 0.54 \mathrm{MGLY}+0.35 \mathrm{ALD}+0.11 \mathrm{KET}+0.12 \mathrm{ACO}_{3} \\
\quad+0.38 \mathrm{HO}_{2}+0.08 \mathrm{XO}_{2}+0.5 \mathrm{MO}_{2}+0.5 \mathrm{ORA} 2\end{array}$ & $7.51 \times 10^{-13}$ & -565 & $5.00 \times 10^{-12}$ & 38 \\
\hline (R208) & $\mathrm{OLNN}+\mathrm{ACO}_{3} \rightarrow \mathrm{ONIT}+0.5 \mathrm{ORA} 2+0.5 \mathrm{MO}_{2}+0.50 \mathrm{HO}_{2}$ & $8.85 \times 10^{-13}$ & -765 & $1.15 \times 10^{-11}$ & 38 \\
\hline (R209) & $\begin{array}{l}\mathrm{OLND}+\mathrm{ACO}_{3} \rightarrow 0.207 \mathrm{HCHO}+0.65 \mathrm{ALD}+0.167 \mathrm{KET}+0.484 \\
\mathrm{ORA} 2+0.484 \mathrm{ONIT}+0.516 \mathrm{NO}_{2}+0.516 \mathrm{MO}_{2}\end{array}$ & $5.37 \times 10^{-13}$ & -765 & $7.00 \times 10^{-12}$ & 38 \\
\hline \multicolumn{6}{|c|}{$\mathrm{NO}_{3}$-Alkene-Peroxyradical $+\mathrm{NO}_{3}$-Alkene-Peroxyradical Reactions } \\
\hline (R210) & $\mathrm{OLNN}+\mathrm{OLNN} \rightarrow 2 \mathrm{ONIT}+\mathrm{HO}_{2}$ & $7.0 \times 10^{-14}$ & -1000 & $2.00 \times 10^{-12}$ & 38 \\
\hline (R211) & $\begin{array}{l}\mathrm{OLNN}+\mathrm{OLND} \rightarrow 0.202 \mathrm{HCHO}+0.64 \mathrm{ALD}+0.149 \mathrm{KET}+0.50 \\
\mathrm{HO}_{2}+1.50 \mathrm{ONIT}+0.50 \mathrm{NO}_{2}\end{array}$ & $4.25 \times 10^{-14}$ & -1000 & $1.22 \times 10^{-12}$ & 38 \\
\hline$(\mathrm{R} 212)$ & $\begin{array}{l}\text { OLND + OLND } \rightarrow 0.504 \mathrm{HCHO}+1.21 \mathrm{ALD}+0.285 \mathrm{KET}+ \\
\text { ONIT }+\mathrm{NO}_{2}\end{array}$ & $2.96 \times 10^{-14}$ & -1000 & $8.50 \times 10^{-13}$ & 38 \\
\hline \multicolumn{6}{|c|}{$\mathrm{NO}_{3}+$ Organic Peroxy Radicals } \\
\hline$(\mathrm{R} 213)$ & $\mathrm{MO}_{2}+\mathrm{NO}_{3} \rightarrow \mathrm{HCHO}+\mathrm{HO}_{2}+\mathrm{NO}_{2}$ & $1.2 \times 10^{-12}$ & & $1.20 \times 10^{-12}$ & 38 \\
\hline (R214) & $\mathrm{ETHP}+\mathrm{NO}_{3} \rightarrow \mathrm{ALD}+\mathrm{HO}_{2}+\mathrm{NO}_{2}$ & $1.20 \times 10^{-12}$ & & $1.20 \times 10^{-12}$ & 38 \\
\hline (R215) & $\begin{array}{l}\mathrm{HC} 3 \mathrm{P}+\mathrm{NO}_{3} \rightarrow 0.048 \mathrm{HCHO}+0.243 \mathrm{ALD}+0.67 \mathrm{KET}+0.063 \mathrm{GLY} \\
\quad+0.792 \mathrm{HO}_{2}+0.155 \mathrm{MO}_{2}+0.053 \mathrm{ETHP}+0.051 \mathrm{XO}_{2}+\mathrm{NO}_{2}\end{array}$ & $1.20 \times 10^{-12}$ & & $1.20 \times 10^{-12}$ & 38 \\
\hline$(\mathrm{R} 216)$ & $\begin{array}{l}\mathrm{HC} 5 \mathrm{P}+\mathrm{NO}_{3} \rightarrow 0.021 \mathrm{HCHO}+0.239 \mathrm{ALD}+0.828 \mathrm{KET}+0.699 \mathrm{HO}_{2} \\
\quad+0.04 \mathrm{MO}_{2}+0.262 \mathrm{ETHP}+0.391 \mathrm{XO}_{2}+\mathrm{NO}_{2}\end{array}$ & $1.20 \times 10^{-12}$ & & $1.20 \times 10^{-12}$ & 38 \\
\hline (R217) & $\begin{array}{l}\mathrm{HC} 8 \mathrm{P}+\mathrm{NO}_{3} \rightarrow 0.187 \mathrm{ALD}+0.88 \mathrm{KET}+0.845 \mathrm{HO}_{2}+0.155 \mathrm{ETHP} \\
\quad+0.587 \mathrm{XO}_{2}+\mathrm{NO}_{2}\end{array}$ & $1.20 \times 10^{-12}$ & & $1.20 \times 10^{-12}$ & 38 \\
\hline (R218) & $\mathrm{ETEP}+\mathrm{NO}_{3} \rightarrow 1.6 \mathrm{HCHO}+0.2 \mathrm{ALD}+\mathrm{HO}_{2}+\mathrm{NO}_{2}$ & $1.20 \times 10^{-12}$ & & $1.20 \times 10^{-12}$ & 38 \\
\hline (R219) & $\mathrm{OLTP}+\mathrm{NO}_{3} \rightarrow \mathrm{HCHO}+0.94 \mathrm{ALD}+0.06 \mathrm{KET}+\mathrm{HO}_{2}+\mathrm{NO}_{2}$ & $1.20 \times 10^{-12}$ & & $1.20 \times 10^{-12}$ & 38 \\
\hline (R220) & $\mathrm{OLIP}+\mathrm{NO}_{3} \rightarrow 1.71 \mathrm{ALD}+0.29 \mathrm{KET}+\mathrm{HO}_{2}+\mathrm{NO}_{2}$ & $1.20 \times 10^{-12}$ & & $1.20 \times 10^{-12}$ & 38 \\
\hline (R221) & $\begin{array}{l}\mathrm{ISOP}+\mathrm{NO}_{3} \rightarrow 0.60 \mathrm{MACR}+0.40 \mathrm{OLT}+0.686 \mathrm{HCHO}+ \\
\mathrm{HO}_{2}+\mathrm{NO}_{2}\end{array}$ & $1.20 \times 10^{-12}$ & & $1.20 \times 10^{-12}$ & 42 \\
\hline (R222) & $\mathrm{APIP}+\mathrm{NO}_{3} \rightarrow \mathrm{ALD}+\mathrm{KET}+\mathrm{HO}_{2}+\mathrm{NO}_{2}$ & $1.20 \times 10^{-12}$ & & $1.20 \times 10^{-12}$ & 9 \\
\hline (R223) & $\mathrm{LIMP}+\mathrm{NO}_{3} \rightarrow 0.60 \mathrm{MACR}+0.40 \mathrm{OLI}+0.40 \mathrm{HCHO}+\mathrm{HO}_{2}+\mathrm{NO}_{2}$ & $1.20 \times 10^{-12}$ & & $1.20 \times 10^{-12}$ & 9 \\
\hline (R224) & $\mathrm{TOLP}+\mathrm{NO}_{3} \rightarrow 0.70 \mathrm{MGLY}+1.30 \mathrm{GLY}+0.50 \mathrm{DCB}+\mathrm{HO}_{2}+\mathrm{NO}_{2}$ & $1.20 \times 10^{-12}$ & & $1.20 \times 10^{-12}$ & 38 \\
\hline$(\mathrm{R} 225)$ & $\mathrm{XYLP}+\mathrm{NO}_{3} \rightarrow 1.26 \mathrm{MGLY}+0.74 \mathrm{GLY}+\mathrm{DCB}+\mathrm{HO}_{2}+\mathrm{NO}_{2}$ & $1.20 \times 10^{-12}$ & & $1.20 \times 10^{-12}$ & 38 \\
\hline (R226) & $\mathrm{CSLP}+\mathrm{NO}_{3} \rightarrow \mathrm{GLY}+\mathrm{MGLY}+\mathrm{HO}_{2}+\mathrm{NO}_{2}$ & $1.20 \times 10^{-12}$ & & $1.20 \times 10^{-12}$ & 38 \\
\hline (R227) & $\mathrm{ACO}_{3}+\mathrm{NO}_{3} \rightarrow \mathrm{MO}_{2}+\mathrm{NO}_{2}$ & $4.00 \times 10^{-12}$ & & $4.00 \times 10^{-12}$ & 38 \\
\hline (R228) & $\mathrm{TCO}_{3}+\mathrm{NO}_{3} \rightarrow \mathrm{HCHO}+\mathrm{ACO}_{3}+\mathrm{NO}_{2}$ & $4.00 \times 10^{-12}$ & & $4.00 \times 10^{-12}$ & 38 \\
\hline (R229) & $\begin{array}{l}\mathrm{KETP}+\mathrm{NO}_{3} \rightarrow 0.54 \mathrm{MGLY}+0.46 \mathrm{AID}+0.77 \mathrm{HO}_{2}+0.23 \mathrm{ACO}_{3}+ \\
0.16 \mathrm{XO}_{2}+\mathrm{NO}_{2}\end{array}$ & $1.20 \times 10^{-12}$ & & $1.20 \times 10^{-12}$ & 38 \\
\hline$(\mathrm{R} 230)$ & $\mathrm{OLNN}+\mathrm{NO}_{3} \rightarrow \mathrm{ONIT}+\mathrm{HO}_{2}+\mathrm{NO}_{2}$ & $1.20 \times 10^{-12}$ & & $1.20 \times 10^{-12}$ & 38 \\
\hline (R231) & $\mathrm{OLND}+\mathrm{NO}_{3} \rightarrow 0.28 \mathrm{HCHO}+1.24 \mathrm{ALD}+0.469 \mathrm{KET}+2 \mathrm{NO}_{2}$ & $1.20 \times 10^{-12}$ & & $1.20 \times 10^{-12}$ & 38 \\
\hline
\end{tabular}


Table 2b. (continued)

\begin{tabular}{|c|c|c|c|c|c|c|}
\hline $\begin{array}{c}\text { Reaction } \\
\text { No. }\end{array}$ & Reaction & & $\underset{\mathrm{cm}^{3} \mathrm{~s}^{-1}}{A,}$ & $\begin{array}{c}E / R \\
\mathrm{~K}\end{array}$ & $k^{\mathrm{a}}$ & Note \\
\hline & & Operator Reactions & & & & \\
\hline (R232) & $\mathrm{XO}_{2}+\mathrm{HO}_{2} \rightarrow \mathrm{OP} 2$ & & $1.66 \times 10^{13}$ & -1300 & $1.30 \times 10^{-11}$ & 38 \\
\hline (R233) & $\mathrm{XO}_{2}+\mathrm{MO}_{2} \rightarrow \mathrm{HCHO}+\mathrm{HO}_{2}$ & & $5.99 \times 10^{-15}$ & -1510 & $9.50 \times 10^{-13}$ & 38 \\
\hline (R234) & $\mathrm{XO}_{2}+\mathrm{ACO}_{3} \rightarrow \mathrm{MO}_{2}$ & & $3.40 \times 10^{-14}$ & -1560 & $6.38 \times 10^{-12}$ & 38 \\
\hline (R235) & $\mathrm{XO}_{2}+\mathrm{XO}_{2} \rightarrow$ & & $7.13 \times 10^{-17}$ & -2950 & $1.42 \times 10^{-12}$ & 38 \\
\hline (R236) & $\mathrm{XO}_{2}+\mathrm{NO} \rightarrow \mathrm{NO}_{2}$ & & $4.0 \times 10^{-12}$ & & $4.00 \times 10^{-12}$ & 38 \\
\hline (R237) & $\mathrm{XO}_{2}+\mathrm{NO}_{3} \rightarrow \mathrm{NO}_{2}$ & & $1.20 \times 10^{-12}$ & & $1.20 \times 10^{-12}$ & 38 \\
\hline
\end{tabular}

Notes 1, DeMore et al. [1994]; 2, products estimated (see text); 3, products from Uselman et al. [1979]; 4, Atkinson and Lloyd [1984]; 5, Paulson et al., [1992a]; 6, rate constant taken to be same as for iso-butene $+O^{3} P$ [Cvetanovic, 1987]; 7, Atkinson [1994]; 8, rate constant and product distribution calculated for average U.S. emissions (see text); 9, Kirchner and Stockwell [1996b]; 10, E/R taken to be same as for the toluene + $\mathrm{HO}$ reaction; 11, taken as $m$-cresol; 12, $\mathrm{O}_{2}$-addition channel [Atkinson, 1994] is ignored for simplification (see text); 13, Tuazon and Atkinson [1990]; 14, rate constant from Bierbach et al. [1994]; 15, rate constant from Wiesen et al. [1995]; 16, rate constant estimated as for propyl hydroperoxide; 17, rate constant as for $\mathrm{CH}_{3} \mathrm{O}_{2} \mathrm{H}+\mathrm{HO} ; 18$, rate constant is upper limit; 19, TPAN treated as methacrylic peroxy nitrate; 20, rate constant from Grosjean et al. [1993a], E/R from Kirchner and Stockwell [1996b], and products from Grosjean et al. [1993b]; 21, rate constant taken to be as $\mathrm{HC} 3$; decomposition is assumed; $22, E / R$ is treated as $\mathrm{ALD}+\mathrm{NO}_{3} ; 23$, rate constant is assumed to be equal to $2 \times k(\mathrm{ALD}+\mathrm{NO})_{3}$ $-k\left(\mathrm{ETH}+\mathrm{NO}_{3}\right) ; 24$, rate constant is treated as ALD $+\mathrm{NO}_{3} ; 25$, rate constant is taken to be as crotonaldehyde [Atkinson, 1994] and $E / R$ and products from Kirchner and Stockwell [1996b]; 26, rate constant is estimated as the mean of a mixture of 50\% methylphenol and 50\% dimethylphenol [Atkinson, 1994]; 27, rate constant is uncertain because of large variation in rate constant for alkenes grouped into model species; 28, Atkinson [1991]; 29, rate constant is the mean of eight measurements, and products are described by Kirchner and Stockwell [1996b]; 30, rate constant taken to be $80 \%$ of estimated rate constant for methacrolein by Kirchner and Stockwell [1996b]; 31, rate constant and products estimated from Atkinson [1994]; 32, rate constant from Atkinson [1994], and the products were estimated from Kirchner and Stockwell [1996b]; 33, rate constant is from constant from Grosjean et al. [1993a], the yield of $\mathrm{HCHO}$ was from Grosjean et al. [1993b], and other products and $E / R$ were estimated by Kirchner and Stockwell [1996b]; 34, rate constant taken to be same as $\mathrm{CH}_{3} \mathrm{O}+\mathrm{NO}_{2}$; 35, rate constant taken to be same as $\mathrm{C}_{6} \mathrm{H}_{5} \mathrm{CH}_{2} \mathrm{O}_{2}+\mathrm{HO}_{2}$ [Le Bras, 1997]; 36, the difference between Bierbach [1994] and Atkinson [1994] was assumed (see text); 37, rate constant same as corresponding reaction for ADDT; 38, Kirchner and Stockwell [1996a]; 39, Lightfoot et al. [1992]; 40, Le Bras [1997]; 41, rate constant taken to be same as $\mathrm{HO}_{2}+\mathrm{CH}_{2}=\mathrm{CHCH}_{2} \mathrm{O}$ [Le Bras, 1997]; 42, rate constant taken to be same as $\mathrm{HCP}+\mathrm{NO}_{3}$.

${ }^{a}$ The rate constants are for $298 \mathrm{~K}$ and $1 \mathrm{~atm}$. The units for rate constants of first-order reactions are $\mathrm{s}^{-1}$; of second-order reactions, $\mathrm{cm}^{3} \mathrm{~s}^{-1}$; and for third-order reactions, $\mathrm{cm}^{6} \mathrm{~s}^{1}$. For second-order reactions the rate constants are given by $k=A \exp [(-E / R) / T]$ unless indicated otherwise.

concentration of $0.2 \mathrm{ppt}$, the $\mathrm{NO}_{3}$ radical self-reaction occurs at a rate that is $2.6 \%$ of the rate of its reaction with $\mathrm{NO}$ :

$$
\mathrm{NO}_{3}+\mathrm{NO}_{3} \rightarrow 2 \mathrm{NO}_{2}+\mathrm{O}_{2}
$$

Some studies have found evidence for the thermal decomposition of $\mathrm{NO}_{3}$ [Johnston et al., 1986; Davidson et al., 1990; Hjorth et al., 1992]; this reaction could be especially important during warm summer nights:

$$
\mathrm{NO}_{3} \rightarrow \mathrm{NO}+\mathrm{O}_{2}
$$

However, these results are contradicted by the studies of Russell et al. [1986] and Davis et al. [1993]. DeMore et al. [1994] rule out (8) on the basis of the results of Davis et al. [1993], who determined that barrier to thermal dissociation is $47.3 \mathrm{kcal}$ $\mathrm{mol}^{-1}$. On this basis, (8) was not included in the new mechanism.

Cantrell et al. [1985] showed that the reaction of $\mathrm{HO}_{2}$ with $\mathrm{NO}_{3}$ was important for nighttime chemistry. Further research by several groups [Hall et al., 1988; Becker et al., 1992; Mellouki et al., 1993] has shown that the rate constant and especially the products for this reaction in the RADM2 mechanism had to be revised. DeMore et al. [1994] recommend a weighted avcragc $\left(3.5 \times 10^{-12} \mathrm{~cm}^{3} \mathrm{~s}^{-1}\right)$ of Hall et al. [1988], Mellouki et al. [1988, 1993], and Becker et al. [1992] for the overall rate constant. This recommendation was adopted. Yields of 0.7 for the HOproducing channel and 0.3 for the $\mathrm{HNO}_{3}$-producing channel were taken from Le Bras [1997]:

$$
\mathrm{HO}_{2}+\mathrm{NO}_{3} \rightarrow 0.3 \mathrm{HNO}_{3}+\mathrm{O}_{2}+0.7 \mathrm{HO}+0.7 \mathrm{NO}_{2}
$$

These yields are consistent with those of Becker et al. [1992] and Mellouki et al. [1988, 1993] within the experimental uncertainties.

Reaction (10) was added for completeness to the RACM mechanism because of nighttime production of HO from (9) and other sources:

$$
\mathrm{HO}+\mathrm{NO}_{3} \rightarrow \mathrm{NO}_{2}+\mathrm{HO}_{2}
$$

In the atmosphere, $\mathrm{N}_{2} \mathrm{O}_{5}$ reacts with water to produce $\mathrm{HNO}_{3}$ primarily through heterogeneous processes:

Table 2c. The RACM Chemical Mechanism: Reaction Rate Constants of the Form $k=T^{2} C \exp (-D / T)$

\begin{tabular}{lllrr}
\hline $\begin{array}{c}\text { Reaction } \\
\text { No. }\end{array}$ & \multicolumn{1}{c}{ Reaction } & $\begin{array}{c}C, \\
\mathrm{~K}^{-2} \mathrm{~cm}^{3} \mathrm{~s}^{-1}\end{array}$ & $D, \mathrm{~K}$ & Note \\
\hline (R61) & $\mathrm{CH}_{4}+\mathrm{HO} \rightarrow \mathrm{MO}_{2}+\mathrm{H}_{2} \mathrm{O}$ & $7.44 \times 10^{-18}$ & 1361 & 7 \\
$(\mathrm{R} 62)$ & $\mathrm{ETH}+\mathrm{HO} \rightarrow \mathrm{ETHP}+\mathrm{H}_{2} \mathrm{O}$ & $1.51 \times 10^{-17}$ & 492 & 7 \\
$(\mathrm{R} 78)$ & $\mathrm{KET}+\mathrm{HO} \rightarrow \mathrm{KETP}+\mathrm{H}_{2} \mathrm{O}$ & $5.68 \times 10^{-18}$ & -92 & 7 \\
$(\mathrm{R} 98)$ & $\mathrm{ETE}+\mathrm{NO}_{3} \rightarrow 0.80 \mathrm{OLNN}+\mathrm{0}+20$ OLND & $4.88 \times 10^{-18}$ & 2282 & 7 \\
\hline
\end{tabular}

Note 7, Atkinson [1994]. 
Table 2d. The RACM Chemical Mechanism: Troe Reactions

\begin{tabular}{llccccr}
\hline $\begin{array}{c}\text { Reaction } \\
\text { No. }\end{array}$ & \multicolumn{1}{c}{ Reaction } & $\begin{array}{c}k_{0}^{300} \\
\mathrm{~cm}^{6} \mathrm{~s}^{-1}\end{array}$ & $n$ & $\begin{array}{c}k_{\infty}^{300}, \\
\mathrm{~cm}^{3} \mathrm{~s}^{-1}\end{array}$ & $m$ & Note \\
\hline $\begin{array}{l}\text { (R35) } \\
(\mathrm{R} 37)\end{array}$ & $\mathrm{O}^{3} \mathrm{P}+\mathrm{NO} \rightarrow \mathrm{NO}_{2}$ & $9.00 \times 10^{-32}$ & 1.5 & $3.00 \times 10^{-11}$ & 0.0 & 1 \\
$(\mathrm{R} 38)$ & $\mathrm{O} P+\mathrm{NO}_{2} \rightarrow \mathrm{NO}_{3}$ & $9.00 \times 10^{-32}$ & 2.0 & $2.20 \times 10^{-11}$ & 0.0 & 1 \\
$(\mathrm{R} 39)$ & $\mathrm{HO}+\mathrm{NO}_{2} \rightarrow \mathrm{HNNO}_{3}$ & $7.00 \times 10^{-31}$ & 2.6 & $1.50 \times 10^{-11}$ & 0.5 & 1 \\
$(\mathrm{R} 42)$ & $\mathrm{HO}_{2}+\mathrm{NO}_{2} \rightarrow \mathrm{HNO}_{4}$ & $2.60 \times 10^{-30}$ & 3.2 & $2.40 \times 10^{-11}$ & 1.3 & 1 \\
$(\mathrm{R} 53)$ & $\mathrm{NO}_{3}+\mathrm{NO}_{2} \rightarrow \mathrm{N}_{2} \mathrm{O}_{5}$ & $2.80 \times 10^{-31}$ & 3.2 & $4.70 \times 10^{-12}$ & 1.4 & 1 \\
$(\mathrm{R} 57)$ & $\mathrm{HO}^{3}+\mathrm{SO}_{2} \rightarrow \mathrm{SULF}^{2}+\mathrm{HO}_{2}$ & $3.00 \times 10^{-30}$ & 3.9 & $1.50 \times 10^{-12}$ & 0.7 & 1 \\
$(\mathrm{R} 127)$ & $\mathrm{ACO}_{3}+\mathrm{NO}_{2} \rightarrow \mathrm{PAN}$ & $9.70 \times 10^{-29}$ & 3.3 & $1.50 \times 10^{-12}$ & 0.0 & 1 \\
$(\mathrm{R} 129)$ & $\mathrm{TCO}_{3}+\mathrm{NO}_{2} \rightarrow$ TPAN & $9.70 \times 10^{-29}$ & 5.6 & $9.30 \times 10^{-12}$ & 1.5 & 1 \\
\hline
\end{tabular}

Here, $k\left(\mathrm{~cm}^{3}\right.$ molecule $\left.^{-1} \mathrm{~s}^{-1}\right)=\left\{k_{0}(T)[M] /\left(1+k_{0}(T)[M] / k_{\infty}(T)\right)\right\} 0.6^{\left\{1+\left[\log _{10}\left(k_{0}(T)[M] / k_{\infty}(T)\right)\right\}^{2}\right\}^{-1}}$ where: $k_{0}(T)=k_{0}^{300}(T / 300)^{-n} ; k_{\infty}(T)=k_{\infty}^{300}(T / 300)^{-m}$; and $[M]$ is the concentration of air in molecules $\mathrm{cm}^{-3}$ [DeMore et al., 1994]. Note 1, DeMore et al. [1994]; 38, Kirchner and Stockwell [1996a, 1997].

$$
\mathrm{N}_{2} \mathrm{O}_{5}+\mathrm{H}_{2} \mathrm{O} \rightarrow 2 \mathrm{HNO}_{3}
$$

However, the gas-phase reaction of $\mathrm{N}_{2} \mathrm{O}_{5}$ with $\mathrm{H}_{2} \mathrm{O}$ is not a fast reaction due to entropy considerations [Calvert and Stockwell, 1983]. Experimental measurements of the gas-phase rate constant are complicated by a strong heterogeneous component to the reaction rate. The rate constant utilized by RADM2, $2.0 \times 10^{-21} \mathrm{~cm}^{3} \mathrm{~s}^{-1}$, is the upper limit recommended by DeMore et al. [1994]. Other workers report an upper limit of $5 \times 10^{-22} \mathrm{~cm}^{3} \mathrm{~s}^{-1}$ [Sverdrup et al., 1987]. Given the entropy considerations and the strong interference of heterogeneous reactions in the available measurements, this reaction was omitted from the RACM mechanism.

\section{Organic Chemistry}

The recent mechanisms of Andersson-Sköld [1995] and Jenkin et al. [1996] include highly detailed descriptions of atmospheric organic oxidation mechanisms. The mechanism of Andersson-Sköld was developed to describe the gas-phase chemistry of 90 emitted compounds, and this mechanism includes more than 700 species with nearly 2000 reactions. The mechanism of Jenkin et al. is even larger with 120 emitted organic compounds reacting in 2500 chemical species and 7000 chemical reactions. However, emissions inventories include hundreds of emitted volatile organic compounds (VOC) [Middleton et al., 1990]. Given the need to conserve computational resources for a transport/transformation model and the complexity of explicit detailed mechanisms, it is necessary to group organic compounds together to form a manageable set of model classes. For the same reason, many multiple pathways are formulated as one reaction in the RACM mechanism, and not all organic intermediates (i.e., alkyl radicals) are explicitly described.

\subsection{Aggregation of Organic Compounds}

For the RACM mechanism the hundreds of VOCs in the real atmosphere are aggregated into 16 anthropogenic and 3 biogenic model species (Table 3). The grouping of organic chemical species into the RACM model species is based on the magnitudes of the emission rates, similarities in functional groups and the compound's reactivity toward HO [Middleton et al., 1990; Stockwell et al., 1990]. The aggregation procedure was done in two steps to simplify the process. First, hundreds of anthropogenic VOC are grouped into 32 emission categories, and second, these categories are aggregated into 16 model species (Table 3). For further details the reader is referred to Middleton et al. [1990] and Stockwell et al. [1990]. Here we focus on our revisions to the procedure. In the text the following subscripts are used: " $i$ " for chemical species, "cat" for Middleton et al. emission categories, and " $m$ " for model species. It should be stressed that the aggregated rate constants presented in Table $2 \mathrm{~b}$ and the aggregation factors presented in Table 3 were calculated on the basis of a specific emissions inventory [Middleton et al., 1990]. The procedure is completely general and can be used to adapt the mechanism's rate constants and the aggregation factors to other inventories. The procedure is illustrated in detail for alkanes, and it is applicable to the other classes of organic compounds as well.

One of the parameters used for the aggregation is the reactivity of the VOC toward HO. The rate constants $k_{m \text {, HO }}$ for the reactions of the model species with $\mathrm{HO}$ were calculated as the weighted mean of the rate constants $k_{\mathrm{cat}, \mathrm{HO}}$ of all categories aggregated together into a single model species (for an example, see Table 4). The rate constants $k_{\mathrm{cat}, \mathrm{HO}}$ of the categories were calculated as the weighted mean of the rate constants $k_{i, \mathrm{HO}}$ at $298 \mathrm{~K}$ of the chemical species grouped into this

Table 2e. The RACM Chemical Mechanism: Troe Equilibrium Reactions

\begin{tabular}{|c|c|c|c|c|c|c|c|c|}
\hline $\begin{array}{c}\text { Reaction } \\
\text { No. }\end{array}$ & Reaction & A & $B$ & $\begin{array}{c}k_{0}^{300} \\
\mathrm{~cm}^{6} \mathrm{~s}^{-1}\end{array}$ & $n$ & $\begin{array}{c}k_{\infty}^{300} \\
\mathrm{~cm}^{3} \mathrm{~s}^{-1}\end{array}$ & $m$ & Note \\
\hline (R43) & $\mathrm{HNO}_{4} \rightarrow \mathrm{HO}_{2}+\mathrm{NO}_{2}$ & $4.76 \times 10^{+26}$ & 10900 & $1.80 \times 10^{-31}$ & 3.2 & $4.70 \times 10^{-12}$ & 1.4 & 1 \\
\hline (R54) & $\mathrm{N}_{2} \mathrm{O}_{5} \rightarrow \mathrm{NO}_{2}+\mathrm{NO}_{3}$ & $3.70 \times 10^{+26}$ & 11000 & $2.20 \times 10^{-30}$ & 3.9 & $1.50 \times 10^{-12}$ & 0.7 & 1 \\
\hline (R128) & $\mathrm{PAN} \rightarrow \mathrm{ACO}_{3}+\mathrm{NO}_{2}$ & $1.16 \times 10^{+28}$ & 13954 & $9.70 \times 10^{-29}$ & 5.6 & $9.30 \times 10^{-12}$ & 1.5 & 38 \\
\hline (R130) & $\mathrm{TPAN} \rightarrow \mathrm{TCO}_{3}+\mathrm{NO}_{2}$ & $1.16 \times 10^{+28}$ & 13954 & $9.70 \times 10^{-29}$ & 5.6 & $9.30 \times 10^{-12}$ & 1.5 & 38 \\
\hline
\end{tabular}

Here, $k\left(\mathrm{~s}^{-1}\right)=A \exp (-\mathrm{B} / \mathrm{T}) \times\left\{k_{0}(T)[M] /\left(1+k_{0}(T)[M] / k_{\infty}(T)\right)\right\} 0.6^{\left\{1+\left[\log _{10}\left(k_{0}(T)[M] / k_{\infty}(T)\right)\right]^{2}\right\}^{-1}}$, where $k_{0}(T), k_{\infty}(T)$, and $[M]$ are as defined for Table $2 \mathrm{f}$ [DeMore et al., 1994]. Notes 1, DeMore et al. [1994]; 38, Kirchner and Stockwell [1996a]. 
Table 2f. The RACM Chemical Mechanism: Reactions With Special Rate Expressions

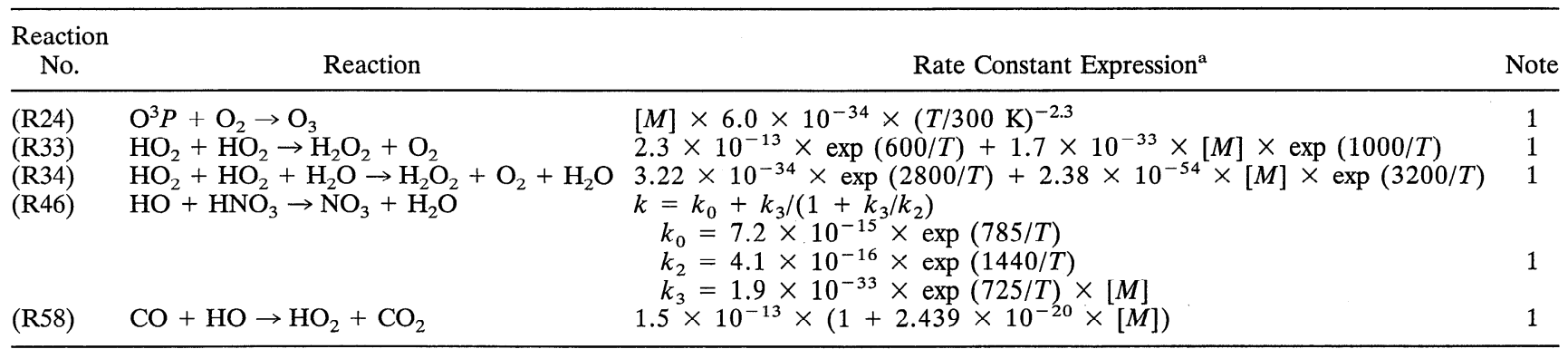

Note 1, DeMore et al. [1994].

The units for second-order rate constants are $\mathrm{cm}^{3} \mathrm{~s}^{-1}$ and for third-order the units are $\mathrm{cm}^{6} \mathrm{~s}^{-1}$. For all of the above, $[M]$ is the concentration of air in molecules $\mathrm{cm}^{-3}$.

Table 3. Allocation of Emissions Categories to RACM Species

\begin{tabular}{|c|c|c|c|}
\hline Category $^{\mathrm{a}}$ & Emission Category Description ${ }^{a}$ & $\begin{array}{l}\text { RACM } \\
\text { Species }\end{array}$ & $\begin{array}{l}\text { Aggregation } \\
\text { Factor }\end{array}$ \\
\hline 1 & methane & $\mathrm{CH} 4$ & 1.00 \\
\hline 2 & ethane & ETH & 1.00 \\
\hline 3 & propane & $\mathrm{HC} 3$ & 0.57 \\
\hline 4 & $\begin{array}{l}\text { alkanes with } \mathrm{HO} \text { rate constant }(298 \mathrm{~K}, 1 \mathrm{~atm}) \text { between } \\
1.7 \times 10^{-12} \text { and } 3.4 \times 10^{-12} \mathrm{~cm}^{3} \mathrm{~s}^{-1}\end{array}$ & $\mathrm{HC} 3$ & 1.11 \\
\hline 5 & $\begin{array}{l}\text { alkanes with HO rate constant }(298 \mathrm{~K}, 1 \mathrm{~atm}) \text { between } \\
3.4 \times 10^{-12} \text { and } 6.8 \times 10^{-12} \mathrm{~cm}^{3} \mathrm{~s}^{-1}\end{array}$ & HC5 & 0.97 \\
\hline 6 & $\begin{array}{l}\text { alkanes with HO rate constant }(298 \mathrm{~K}, 1 \mathrm{~atm}) \text { between } \\
6.8 \times 10^{-12} \text { and } 1.36 \times 10^{-11} \mathrm{~cm}^{3} \mathrm{~s}^{-1}\end{array}$ & $\mathrm{HC} 8$ & 0.94 \\
\hline 7 & $\begin{array}{l}\text { alkanes with HO rate constant }(298 \mathrm{~K}, 1 \mathrm{~atm}) \text { greater } \\
\text { than } 1.36 \times 10^{-11} \mathrm{~cm}^{3} \mathrm{~s}^{-1}\end{array}$ & $\mathrm{HC} 8$ & 1.14 \\
\hline \multirow[t]{2}{*}{8} & alkane/aromatic mix & $\mathrm{HC} 8$ & 0.91 \\
\hline & & XYL & 0.09 \\
\hline 9 & ethene & ETE & 1.00 \\
\hline 10 & propene & OLT & 1.00 \\
\hline 11 & alkenes (primary) & OLT & 1.00 \\
\hline 12 & alkenes (internal) & OLI & 1.00 \\
\hline \multirow[t]{2}{*}{13} & alkenes (primary/internal mix) & OLI & 0.50 \\
\hline & & OLT & 0.50 \\
\hline 14 & benzene, halobenzenes & TOL & 0.29 \\
\hline 15 & $\begin{array}{l}\text { aromatics with HO rate constant }(298 \mathrm{~K}, 1 \mathrm{~atm}) \\
\text { less than } 1.36 \times 10^{-11} \mathrm{~cm}^{3} \mathrm{~s}^{-1}\end{array}$ & TOL & 1.00 \\
\hline 16 & $\begin{array}{l}\text { aromatics with } \mathrm{HO} \text { rate constant }(298 \mathrm{~K}, 1 \mathrm{~atm}) \\
\text { greater than } 1.36 \times 10^{-11} \mathrm{~cm}^{3} \mathrm{~s}^{-1}\end{array}$ & XYL & 1.00 \\
\hline 17 & phenols and cresols & CSL & 1.00 \\
\hline \multirow[t]{2}{*}{18} & styrenes & OLT & 1.00 \\
\hline & & TOL & 1.00 \\
\hline 19 & formaldehyde & HCHO & 1.00 \\
\hline 20 & higher aldehydes & ALD & 1.00 \\
\hline 21 & acetone & KET & 0.33 \\
\hline 22 & higher ketones & KET & 1.61 \\
\hline 23 & organic acids ${ }^{\mathrm{b}}$ & ORA2 & 1.00 \\
\hline 24 & acetylene & $\mathrm{HC} 3$ & 0.41 \\
\hline 25 & haloalkenes & $\mathrm{HC} 3$ & 0.44 \\
\hline 26 & unreactive & & \\
\hline 27 & $\begin{array}{l}\text { others with } \mathrm{HO} \text { rate constant }(298 \mathrm{~K}, 1 \mathrm{~atm}) \\
\text { less than } 1.7 \times 10^{-12} \mathrm{~cm}^{3} \mathrm{~s}^{-1}\end{array}$ & $\mathrm{HC} 3$ & 0.49 \\
\hline 28 & $\begin{array}{l}\text { others with } \mathrm{HO} \text { rate constant }(298 \mathrm{~K}, 1 \mathrm{~atm}) \\
\text { between } 1.7 \times 10^{-12} \text { and } 3.4 \times 10^{-12} \mathrm{~cm}^{3} \mathrm{~s}^{-1}\end{array}$ & $\mathrm{HC} 3$ & 1.37 \\
\hline 29 & $\begin{array}{l}\text { others with HO rate constant }(298 \mathrm{~K}, 1 \mathrm{~atm}) \\
\text { between } 3.4 \times 10^{-12} \text { and } 6.8 \times 10^{-12} \mathrm{~cm}^{3} \mathrm{~s}^{-1}\end{array}$ & HC5 & 1.07 \\
\hline 30 & $\begin{array}{l}\text { others with HO rate constant }(298 \mathrm{~K}, 1 \mathrm{~atm}) \\
\text { greatcr than } 6.8 \times 10^{-12} \mathrm{~cm}^{3} \mathrm{~s}^{-1}\end{array}$ & $\mathrm{HC} 8$ & 1.15 \\
\hline 31 & unidentified $^{\mathrm{c}}$ & & \\
\hline 32 & unassigned $^{\mathrm{c}}$ & & \\
\hline
\end{tabular}

${ }^{a}$ Emission inventory and definition of emission categories taken from Middleton et al. [1990].

${ }^{\text {b}}$ There was no separate category for formic acid emissions given by Middleton et al. [1990]. If formic acid emissions are available, they should be grouped into ORA1 with an aggregation factor of 1.00 .

'Not assigned to any model species. 
Table 4. Allocation of Alkane Emissions to RACM Species

\begin{tabular}{|c|c|c|c|c|c|c|c|}
\hline \multirow[b]{2}{*}{$\begin{array}{l}\text { Model } \\
\text { Species }\end{array}$} & \multirow[b]{2}{*}{$\begin{array}{l}\text { Categories }{ }^{\mathrm{a}} \text { and } \\
\text { Chemical Species }^{\mathrm{b}}\end{array}$} & \multicolumn{2}{|c|}{ Chemical Species } & \multicolumn{3}{|c|}{ Category } & \multirow{2}{*}{$\begin{array}{c}\text { Model Species } \\
k_{m, \mathrm{HO}} \\
10^{-12} \mathrm{~cm}^{-3} \mathrm{~s}^{-1}\end{array}$} \\
\hline & & $\begin{array}{l}\text { Emission Rate }^{\mathrm{c}} \\
\mathrm{Mmol} \mathrm{yr}^{-1}\end{array}$ & $10^{-12} \mathrm{~km}_{i, \mathrm{HO}} \stackrel{\mathrm{d}}{\mathrm{cm}^{-3}} \mathrm{~s}^{-1}$ & $\begin{array}{c}\text { Percent Emission, }{ }^{\circ} \\
\% E_{\text {cat }}\end{array}$ & 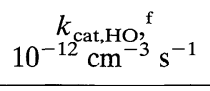 & $\begin{array}{l}\text { Aggregation } \\
\text { Factor }^{\mathrm{g}}\end{array}$ & \\
\hline \multicolumn{8}{|l|}{$\mathrm{CH} 4$} \\
\hline & category 1 & & & 100 & 0.007 & 1.00 & \\
\hline & $\begin{array}{l}\text { methane } \\
\text { CH4 aggregated rate constant }\end{array}$ & & 0.007 & & & & 0.007 \\
\hline \multirow[t]{3}{*}{ ETH } & & & & & & & \\
\hline & $\begin{array}{l}\text { category } 2 \\
\text { ethane }\end{array}$ & & 0.257 & 100 & 0.257 & 1.00 & \\
\hline & ETH aggregated rate constant & & & & & & 0.257 \\
\hline \multirow[t]{21}{*}{$\mathrm{HC} 3$} & & & & & & & \\
\hline & category 3 & & & 4.8 & 1.15 & 0.57 & \\
\hline & propane & 2,818 & 1.15 & & & & \\
\hline & category 4 & & & 40.4 & 2.49 & 1.11 & \\
\hline & $\begin{array}{l}n \text {-butane } \\
i \text {-butane }\end{array}$ & 18,672 & 2.54 & & & & \\
\hline & & 5,069 & 2.33 & & & & \\
\hline & $\begin{array}{l}\text { 2,2-dimethylbutane } \\
\text { category } 24\end{array}$ & 233 & 2.32 & 236 & 081 & 0.41 & \\
\hline & acetylene & 14,000 & 0.81 & 23.6 & 0.01 & 0.41 & \\
\hline & category 27 & & & 3.1 & 0.99 & 0.49 & \\
\hline & methanol & 1,250 & 0.94 & & & & \\
\hline & ethylacetate & 386 & $1.48^{\mathrm{h}}$ & & & & \\
\hline & methylacetate & 203 & $0.35^{\mathrm{h}}$ & & & & \\
\hline & category 28 & 200 & & 28.2 & 3.27 & 1.37 & \\
\hline & ethanol & 14,978 & 3.27 & & & & \\
\hline & isopropyl acetate & 559 & $3.72^{\mathrm{h}}$ & & & & \\
\hline & dimethylether & 1,217 & 2.98 & & & & \\
\hline & category 25 & & & $0^{\mathrm{i}}$ & 0.88 & 0.44 & \\
\hline & perchlorethylene & 2,129 & 0.17 & & & & \\
\hline & trichloroethylene & 1,012 & 1.75 & & & & \\
\hline & ethylene dichloride & 371 & 2.61 & & & & \\
\hline & HC3 aggregated rate constant & & & & & & 2.20 \\
\hline \multirow{14}{*}{$\mathrm{HC} 5$} & category 5 & & & 69.8 & 4.54 & 0.97 & \\
\hline & $i$-pentane & $668^{\mathrm{j}}$ & 3.9 & & & & \\
\hline & hexane & $380^{\mathrm{k}}$ & 5.61 & & & - & \\
\hline & $n$-pentane & $377^{j}$ & 3.94 & & & & \\
\hline & 2-methylpentane & $191^{\mathrm{k}, 1}$ & 5.6 & & & & \\
\hline & 3-methyl pentane & $119^{\mathrm{k}, 1}$ & 5.7 & & & & \\
\hline & 2,2,4-trimethylpentane & 161 & 3.59 & & & & \\
\hline & 2,3-dimethylbutane & 86 & 5.99 & & & & \\
\hline & cyclopentane & $38^{\mathrm{j}}$ & 5.08 & & & & \\
\hline & category 29 & & & 30.2 & 5.29 & 1.07 & \\
\hline & isopropanol & 538 & 5.32 & & & & \\
\hline & $n$-butyl acetate & 180 & $5.71^{\mathrm{m}}$ & & & & \\
\hline & $n$-propyl acetate & 50 & $3.42^{\mathrm{m}}$ & & & & \\
\hline & HC5 aggregated rate constant & & & & & & 4.77 \\
\hline \multirow[t]{23}{*}{$\mathrm{HC} 8$} & category 6 & & & 81.4 & 9.21 & 0.94 & \\
\hline & heptane & 456 & 7.15 & & & & \\
\hline & methylcyclohexane & $322^{\mathrm{n}}$ & 10.4 & & & & \\
\hline & $n$-decane & $208^{\circ}$ & 11.6 & & & & \\
\hline & nonane & $152^{\mathrm{p}}$ & 10.2 & & & & \\
\hline & 2,4-dimethylhexane & 110 & $8.57^{\mathrm{q}}$ & & & & \\
\hline & methylcyclopentane & 105 & $11.66^{\mathrm{q}}$ & & & & \\
\hline & octane & 98 & 8.68 & & & & \\
\hline & 2,3,3-trimethylpentane & 90 & $4.38^{\mathrm{q}}$ & & & & \\
\hline & 3-methylhexane & $114^{\mathrm{r}}$ & $10.1^{\mathrm{q}}$ & & & & \\
\hline & $n$-undecane & 82 & 13.2 & & & & \\
\hline & cyclohexane & 71 & 7.49 & & & & \\
\hline & 2,4-dimethylpentane & 67 & 5.2 & & & & \\
\hline & 2,5-dimethylheptane & 59 & $9.98^{\mathrm{q}}$ & & & & \\
\hline & 3,4-dimethyloctane & 59 & $11.68^{\mathrm{q}}$ & & & & \\
\hline & dimethylcyclohexane & 40 & $11.88^{\mathrm{q}}$ & & & & \\
\hline & 2,2,5-trimethylhexane & 39 & $13.09^{\mathrm{q}}$ & & & & \\
\hline & 4-methylheptane & 38 & $8.57^{\mathrm{q}}$ & & & & \\
\hline & 3-methylheptane & 32 & $8.57^{\mathrm{q}}$ & & & & \\
\hline & 2,4,5-trimethylheptane & 21 & $13.09^{\mathrm{q}}$ & & & & \\
\hline & category 7 & & & 10.8 & 17.34 & 1.14 & \\
\hline & $n$-pentadecane & 226 & 22 & & & & \\
\hline & 2-methyldecane & $116^{\mathrm{s}}$ & $12.52^{q}$ & & & & \\
\hline
\end{tabular}


Table 4. (continued)

\begin{tabular}{|c|c|c|c|c|c|c|c|}
\hline \multirow[b]{2}{*}{$\begin{array}{l}\text { Model } \\
\text { Species }\end{array}$} & \multirow[b]{2}{*}{$\begin{array}{c}\text { Categories }^{\mathrm{a}} \text { and } \\
\text { Chemical Species }^{\mathrm{b}}\end{array}$} & \multicolumn{2}{|c|}{ Chemical Species } & \multicolumn{3}{|c|}{ Category } & \multirow{2}{*}{$\begin{array}{c}\text { Model Species } \\
k_{m, \mathrm{HO}} \\
10^{-12} \mathrm{~cm}^{-3} \mathrm{~s}^{-1}\end{array}$} \\
\hline & & $\begin{array}{c}\text { Emission Rate, } \\
\mathrm{Mmol} \mathrm{yr}^{\mathrm{c}}\end{array}$ & $\underset{10^{-12} \mathrm{~cm}_{i, \mathrm{HO}}{ }^{\mathrm{d}}}{\mathrm{cm}^{-1}}$ & $\begin{array}{c}\text { Percent Emission, } \\
\% E_{\text {cat }}\end{array}$ & $\begin{array}{c}k_{\text {cat,HO }}, \stackrel{f}{f}, \\
10^{-12} \mathrm{~cm}^{-3} \mathrm{~s}^{-1}\end{array}$ & $\begin{array}{l}\text { Aggregation } \\
\text { Factor }\end{array}$ & \\
\hline & diethylcyclohexane & 48 & $15.53^{\mathrm{q}}$ & \multirow{9}{*}{7.8} & \multirow{9}{*}{18.16} & \multirow{9}{*}{1.15} & \\
\hline & $n$-dodecane & 40 & 14.2 & & & & \\
\hline & $n$-tridecane & 23 & 16 & & & & \\
\hline & $n$-tetradecane & 21 & 19 & & & & \\
\hline & category 30 & & & & & & \\
\hline & glycol ether & 102 & $26.8^{\mathrm{t}}$ & & & & \\
\hline & propylene glycole & 56 & $13.21^{q}$ & & & & \\
\hline & ethylene glycole & 19 & $5.71^{\mathrm{m}}$ & & & & \\
\hline & HC8 aggregated rate constant & & & & & & 10.8 \\
\hline
\end{tabular}

${ }^{a}$ Category defined by Middleton et al. [1990].

b Species used to calculate $k_{\text {cat,Ho }}$ and the aggregation factor. Species with emissions less than $20 \mathrm{Gg} / \mathrm{yr}$ were usually neglected.

${ }^{\mathrm{c}}$ As from Middleton et al. [1990].

${ }^{\mathrm{d}} \mathrm{HO}$ rate constant at $298 \mathrm{~K}$, from Atkinson [1994] if not mentioned otherwise.

epercent category emission into RACM, percent contribution by moles of the category to the RACM class.

${ }^{\text {f }} \mathrm{HO}$ rate constant of the category at $298 \mathrm{~K}$, calculated according to equation (14).

${ }^{\mathrm{g}}$ Calculated according to equation (16).

${ }_{\mathrm{h}}^{\mathrm{h}}$ Wallington et al. [1988].

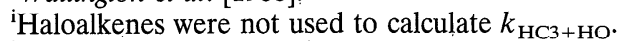

jThe emissions of "isomers of pentane" and "C5 paraffin" were interpreted as $62 \% i$-pentane, $35 \%$ n-pentane, and $3 \%$ cyclopentane corresponding to the emissions of these species.

kThe emissions of "isomers hexane" were interpreted as 55\% hexane, 28\% 2-methylpentane, and 17\% 3-methylpentane corresponding to the emissions of these species.

'The emissions of "methylpentane" were interpreted as $62 \%$ 2-methylpentane and 38\% 3-methylpentane corresponding to the emissions of these species.

${ }^{\mathrm{m}}$ Williams et al. [1993].

"The emissions of "C7-cycloparaffins" were interpreted as methylcyclohexane.

"The emissions of "isomers of decane" were interpreted as $n$-decane.

pThe emissions of "isomers of nonane" were interpreted as nonane.

${ }^{\mathrm{q}}$ Calculated using Kwok and Atkinson [1995].

rThe emissions of "methylhexane" were interpreted as 3-methylhexane.

sThe emissions of "isomers of undecane" were interpreted as 2-methyldecane.

${ }^{\mathrm{t}}$ Dagaut et al. [1989].

category. The weighting was done on the basis of the emissions $E$ in units of moles per year taken from the U.S. emissions inventory [Middleton et al., 1990]:

$$
k_{m, \mathrm{HO}}=\frac{\sum_{\mathrm{cat} \in m}\left(E_{\mathrm{cat}} k_{\mathrm{cat}, \mathrm{HO}}\right)}{\sum_{\mathrm{cat} \in m} E_{\mathrm{cat}}}=\frac{\sum_{\mathrm{cat} \in m}\left(\% E_{\mathrm{cat}} k_{\mathrm{cat}, \mathrm{HO}}\right)}{100}
$$

with

$$
\begin{gathered}
E_{\text {cat }}=\sum_{i \in \text { cat }} E_{i}, \quad \% E_{\text {cat }}=100 \frac{E_{\text {cat }}}{\sum_{\text {cat }} E_{\text {cat }}} \\
k_{\text {cat }, \mathrm{HO}}=\frac{\sum_{i \in \text { cat }}\left(E_{i} k_{i, \mathrm{HO}}\right)}{\sum_{i \in \mathrm{cat}} E_{i}}
\end{gathered}
$$

The rate constant was calculated for 273 and $298 \mathrm{~K}$ and fitted to an Arrhenius equation to determine the activation energy of the rate constant for the model species.

The method of reactivity weighting was used to account for the differences in reactivities between chemical species and model species. The reactivity weighting was based on the assumption that the effect of an emitted chemical species on a simulation is approximately proportional to the amount of the compound that reacts with $\mathrm{HO}$ on a daily basis [Stockwell et al., 1990]. Under this assumption an emitted compound can be represented by a model species which reacts at a different rate provided that an aggregation factor, $\operatorname{Agg}_{i}$, is applied to the compound emissions. The factor is the ratio of the fraction of the chemical species $i$ which reacts with $\mathrm{HO}$ during the day to the fraction of the model species $m$ which reacts:

$$
\operatorname{Agg}_{i}=\frac{1-\exp \left(-k_{i, \mathrm{HO}} \int[\mathrm{HO}] d t\right)}{1-\exp \left(-k_{m, \mathrm{HO}} \int[\mathrm{HO}] d t\right)}
$$

For the daily average integrated HO radical concentration, $\int[\mathrm{HO}] d t$, a value of $1.63 \times 10^{11} \mathrm{~s} \mathrm{~cm}^{-3}$ was used [Stockwell et al., 1990]. The aggregation factor becomes unity if both the model and emitted VOC are highly reactive.

To use this aggregation procedure, it is necessary to know the $\mathrm{HO}$ rate constants of every chemical species that is to be aggregated in a model species. However, for cases where this rate constant is not known we calculated aggregation factors for categories, Agg $_{\text {cat }}$. Because species in a category react similarly, the aggregation factors of categories, Agg $_{\text {cal }}$, can be used as an approximation for the aggregation factors of chemical species, Agg $_{i}$ belonging to a category. Table 3 shows the aggregation factors, calculated using 


$$
\operatorname{Agg}_{\mathrm{cat}}=\frac{1-\exp \left(-k_{\mathrm{cat}, \mathrm{HO}} \int[\mathrm{HO}] d t\right)}{1-\exp \left(-k_{m, \mathrm{HO}} \int[\mathrm{HO}] d t\right)} \times M_{\mathrm{cat}}
$$

where $M_{\text {cat }}$ is the mixing factor, which is 1 for categories which belong only to one model species and $1 / n$ for categories which belong to $n$ model species. The rate constants used to calculate $k_{\text {cat,HO }}$ were taken from the recommendations of Atkinson [1994] or calculated according to the procedure of Kwok and Atkinson [1995] by using a structure-reactivity relationship. As an example of the aggregation process, Table 4 shows the complete set of rate constants and aggregation factors for chemical species, classes, and model species for alkanes.

Middleton et al. [1990] is not followed for anthropogenic emissions of isoprene and terpenes. Although isoprene is a terminal alkene, it is treated as the separate model compound, ISO, because it is primarily emitted from biogenic rather than anthropogenic sources. Any anthropogenic emissions of isoprene and terpenes should be aggregated together into the primarily biogenic model species.

\subsection{Alkanes}

Alkanes are very important chemical species which may be transported over long distances. Methane and ethane are treated explicitly in both the RADM2 and RACM mechanisms. All other alkanes along with alkynes, alcohols, esters, and epoxides are aggregated according to their $\mathrm{HO}$ rate constants into three additional model species, HC3, HC5, and $\mathrm{HC}$. HC3 represents species with relatively low $\mathrm{HO}$ reaction rate constants (lower than $3.4 \times 10^{-12} \mathrm{~cm}^{3} \mathrm{~s}^{-1}$ ); HC5 represents species with moderate $\mathrm{HO}$ reaction rate constants (between $3.4 \times 10^{-12}$ and $6.8 \times 10^{-12} \mathrm{~cm}^{3} \mathrm{~s}^{-1}$ ); and HC8 represents species with relatively high $\mathrm{HO}$ reaction rate constants $\left(>6.8 \times 10^{-12} \mathrm{~cm}^{3} \mathrm{~s}^{-1}\right)$.

The determination of the reaction products and product yields of the model species $\mathrm{HC} 3, \mathrm{HC} 5$, and $\mathrm{HC} 8$ is described in the following. Alkanes react with $\mathrm{HO}$ by the abstraction of hydrogen atoms. The product of this reaction is an alkyl radical, which under atmospheric conditions reacts immediately with $\mathrm{O}_{2}$ to form an alkyl peroxy radical:

$$
\begin{gathered}
\mathrm{RH}+\mathrm{HO} \rightarrow \mathrm{R} \cdot+\mathrm{H}_{2} \mathrm{O} \\
\mathrm{R} \cdot+\mathrm{O}_{2} \rightarrow \mathrm{RO}_{2}
\end{gathered}
$$

For example, $n$-butane:

$$
\begin{gathered}
\mathrm{CH}_{3} \mathrm{CH}_{2} \mathrm{CH}_{2} \mathrm{CH}_{3}+\mathrm{HO}\left(+\mathrm{O}_{2}\right) \rightarrow 0.13 \mathrm{CH}_{3} \mathrm{CH}_{2} \mathrm{CH}_{2} \mathrm{CH}_{2} \mathrm{O}_{2} \cdot \\
+0.87 \mathrm{CH}_{3} \mathrm{CH}_{2}\left(\mathrm{CHO}_{2} \cdot\right) \mathrm{CH}_{3}+\mathrm{H}_{2} \mathrm{O}
\end{gathered}
$$

However, alcohols and alkynes, which are grouped together with alkanes in the model species $\mathrm{HC} 3, \mathrm{HC} 5$, and $\mathrm{HC}$, do not always form peroxy radicals but may form other species directly. For that reason the reactions of $\mathrm{HC} 3, \mathrm{HC} 5$, and $\mathrm{HC} 8$ with $\mathrm{HO}$ form products like ALD, GLY, or CO besides HC3P, HC5P, and HC8P, respectively. The product yield fractions, $\alpha_{m, \mathrm{HO}}^{p}$, of the reaction of model species $m$ with HO giving product $p$ were calculated as the weighted mean of the product yields of all chemical species belonging to that model species:

$$
\alpha_{m, \mathrm{HO}}^{p}=\frac{\sum_{i \in m} \operatorname{Agg}_{i} E_{i} \beta_{i, \mathrm{HO}}^{p}}{\sum_{i \in m} \operatorname{Agg}_{i} E_{i}}
$$

where $\beta_{i, \text { HO }}^{p}$ is the product yield fraction of the reaction of the chemical species $i$ with $\mathrm{HO}$ giving the product $p$. The values for $\beta_{i, \mathrm{HO}}^{p}$ were taken from the recommendations of Atkinson [1994] or calculated according to the procedure of Kwok and Atkinson [1995]. For example, $\beta_{\mathrm{CII}_{3} \mathrm{CII}_{2} \mathrm{OH}, \mathrm{HO}}^{\mathrm{ALD}} 0.95$ because

$$
\begin{gathered}
\mathrm{CH}_{3} \mathrm{CH}_{2} \mathrm{OH}+\mathrm{HO} \rightarrow 5 \% \cdot \mathrm{O}_{2} \mathrm{CH}_{2} \mathrm{CH}_{2} \mathrm{OH} \\
+95 \%\left(\mathrm{CH}_{3} \mathrm{CHO}+\mathrm{HO}_{2}\right)+\mathrm{H}_{2} \mathrm{O}
\end{gathered}
$$

\subsection{Peroxy Radical Formed From Alkanes}

The species $\mathrm{CH}_{4}, \mathrm{ETH}, \mathrm{HC} 3, \mathrm{HC} 5$, and $\mathrm{HC} 8$ react with $\mathrm{HO}$ and $\mathrm{O}_{2}$ to form the peroxy radicals $\mathrm{MO}_{2}$, ETHP, HC3P, HC5P, and $\mathrm{HC} 8 \mathrm{P}$, respectively. The composition of the model peroxy radicals $\mathrm{HC} 3 \mathrm{P}, \mathrm{HC} 5 \mathrm{P}$, and $\mathrm{HC} 8 \mathrm{P}$ is determined by the composition of the model species $\mathrm{HC} 3, \mathrm{HC} 5$, and $\mathrm{HC} 8$ and the reactivity of the chemical species which are aggregated in them. In general, organic peroxy radicals $\mathrm{RO}_{2} \cdot$ can react with $\mathrm{NO}, \mathrm{NO}_{2}, \mathrm{NO}_{3}, \mathrm{HO}_{2}$, and $\mathrm{R}^{\prime} \mathrm{O}_{2}$.

$$
\begin{gathered}
\mathrm{RO}_{2} \cdot+\mathrm{NO} \rightarrow x \mathrm{RONO}_{2}+(1-x)\left(\mathrm{RO} \cdot+\mathrm{NO}_{2}\right) \\
\mathrm{RO}_{2} \cdot+\mathrm{NO}_{2} \rightarrow \mathrm{RO}_{2} \mathrm{NO}_{2} \\
\mathrm{RO}_{2} \cdot+\mathrm{NO}_{3} \rightarrow \mathrm{RO} \cdot+\mathrm{NO}_{2}+\mathrm{O}_{2} \\
\mathrm{RO}_{2} \cdot+\mathrm{HO}_{2} \rightarrow \mathrm{ROOH}+\mathrm{O}_{2} \\
\mathrm{RO}_{2} \cdot+\mathrm{R}^{\prime} \mathrm{O}_{2} \cdot \rightarrow \text { products }
\end{gathered}
$$

The determination of the rate constants for (22)-(26) are discussed by Kirchner and Stockwell [1996a].

The alkoxy radicals RO- formed in (22) and (24) may react with $\mathrm{O}_{2}$ or undergo isomerization or decompose. When an RO- radical reacts with $\mathrm{O}_{2}$, an $\mathrm{HO}_{2}$ radical and a carbonyl species are produced. An example for $n$-butane is shown by

$$
\mathrm{CH}_{3} \mathrm{CH}_{2} \mathrm{CH}_{2} \mathrm{CH}_{2} \mathrm{O}_{2} \cdot+\mathrm{NO} \rightarrow \mathrm{CH}_{3} \mathrm{CH}_{2} \mathrm{CH}_{2} \mathrm{CH}_{2} \mathrm{O} \cdot+\mathrm{NO}_{2}
$$

$$
\mathrm{CH}_{3} \mathrm{CH}_{2} \mathrm{CH}_{2} \mathrm{CH}_{2} \mathrm{O} \cdot+\mathrm{O}_{2} \rightarrow \mathrm{CH}_{3} \mathrm{CH}_{2} \mathrm{CH}_{2} \mathrm{CHO}+\mathrm{HO}_{2}
$$

Alkoxy radicals may also decompose to produce a carbonyl species and after addition of $\mathrm{O}_{2}$, a new organic peroxy radical is produced:

$$
\mathrm{CH}_{3} \mathrm{CH}_{2} \mathrm{C}(\mathrm{O} \cdot) \mathrm{HCH}_{3}\left(+\mathrm{O}_{2}\right) \rightarrow \mathrm{CH}_{3} \mathrm{CHO}+\mathrm{CH}_{3} \mathrm{CH}_{2} \mathrm{OO} \cdot
$$

or they can undergo a 1,5-H-isomerization leading normally to an $\mathrm{HO}_{2}$ and a carbonyl species with one additional $\mathrm{NO}$ to $\mathrm{NO}_{2}$ conversion:

$$
\begin{aligned}
& \mathrm{CH}_{3} \mathrm{CH}_{2} \mathrm{CH}_{2} \mathrm{CH}_{2} \mathrm{O} \cdot \rightarrow \mathrm{HOCH}_{2} \mathrm{CH}_{2} \mathrm{CH}_{2} \mathrm{CH}_{2} \cdot \\
& \mathrm{HOCH}_{2} \mathrm{CH}_{2} \mathrm{CH}_{2} \mathrm{CH}_{2} \cdot+\mathrm{O}_{2} \rightarrow \mathrm{HOCH}_{2} \mathrm{CH}_{2} \mathrm{CH}_{2} \mathrm{CH}_{2} \mathrm{O}_{2} \cdot \\
& \mathrm{HOCH}_{2} \mathrm{CH}_{2} \mathrm{CH}_{2} \mathrm{CH}_{2} \mathrm{O}_{2} \cdot+\mathrm{NO} \\
& \rightarrow \mathrm{HOCH}_{2} \mathrm{CH}_{2} \mathrm{CH}_{2} \mathrm{CH}_{2} \mathrm{O} \cdot+\mathrm{NO}_{2} \\
& \mathrm{HOCH}_{2} \mathrm{CH}_{2} \mathrm{CH}_{2} \mathrm{CH}_{2} \mathrm{O} \cdot+\mathrm{O}_{2} \\
& \rightarrow \mathrm{HOCH}_{2} \mathrm{CH}_{2} \mathrm{CH}_{2} \mathrm{CHO}+\mathrm{HO}_{2}
\end{aligned}
$$

In the RACM mechanism, production of the operator radical, $\mathrm{XO}_{2}$, accounts for the additional $\mathrm{NO}$ to $\mathrm{NO}_{2}$ conversation step due to isomerization. For individual alkoxy radical species the rate constants for isomerization of the alkoxy radicals were determined, and these werc uscd to determine the appropriate 
$\mathrm{XO}_{2}$ yield from the average composition of each lumped alkoxy radical species [Kirchner and Stockwell, 1996a].

The calculation of the product yields of the model peroxy radical are based on the product yields of the chemical species in these reactions. Given a general reaction of a model peroxy radical $\mathrm{RO}_{2}(m)$ with a species $\mathrm{X}$, with $\mathrm{X}$ being $\mathrm{NO}, \mathrm{NO}_{2}, \mathrm{NO}_{3}$, $\mathrm{HO}_{2}$, or $\mathrm{R}^{\prime} \mathrm{O}_{2}(m)$ :

$$
\mathrm{RO}_{2}(m)+\mathrm{X} \rightarrow \alpha_{\mathrm{RO}_{2}(m), \mathrm{X}}^{p} P+\alpha_{\mathrm{RO}_{2}(m), \mathrm{X}}^{p^{\prime}} P^{\prime}+\cdots
$$

the product yield fraction, $\alpha_{\mathrm{RO}_{2}(m), X}^{p}$, for a product $p$ from this reaction is given by the weighted mean of the product yield fractions, $\beta_{i, \mathrm{HO}, \mathrm{X}}^{p}$, for a product $p$ from the reaction of the chemical species $i$ with $\mathrm{HO}$ giving an peroxy radical and the subsequent reaction of this species peroxy radical with $\mathrm{X}$ :

$$
\alpha_{\mathrm{RO}_{2}(m), \mathrm{X}}=\frac{\sum_{i \in m} \operatorname{Agg}_{i} E_{i} \beta_{i, \mathrm{HO}, \mathrm{X}}^{p}}{\sum_{i \in m} \operatorname{Agg}_{i} E_{i}}
$$

where $\beta_{i, \mathrm{HO}}^{p}$, is normalized to account only for reaction products which come from the formation of an $\mathrm{RO}_{2}$. Therefore

$$
\sum_{p} \beta_{i, \mathrm{HO}, \mathrm{X}}^{p}=1 \quad \forall i, \mathrm{X}
$$

Here $\beta_{i, \mathrm{HO}, \mathrm{X}}^{p}$ was calculated using product yield of the reactions $\mathrm{R} \cdot+\mathrm{HO}, \mathrm{RO}_{2} \cdot+\mathrm{X}$ and $\mathrm{RO} \cdot$ decomposition. If no product yields were available from laboratory studies, they were calculated following the procedure proposed by Kwok and Atkinson [1995].

\subsection{Carbonyls}

The carbonyl species in the mechanism include formaldehyde (HCHO), acetaldehyde and higher saturated aldehydes (ALD), acetone and higher saturated ketones (KET), unsaturated dicarbonyls (DCB), glyoxal (GLY), and methylglyoxal and other species of the form RC(O)CHO (MGLY). DCB and GLY are produced through the photooxidation of aromatic compounds. Most MGLY is produced through aromatic oxidation and some is produced through ketone reactions.

The reactions of $\mathrm{HCHO}$ are represented by an explicit set of reactions. The chemistry of ALD is treated as acetaldehyde. The chemistry of KET is treated as a mixture of $50 \%$ acetone and 50\% methyl ethyl ketone (by mole fraction). Although methyl ethyl ketone and higher ketones are the main photochemical oxidation products of VOC, the emissions of acetone are twice as high as methyl ethyl ketone on a mole basis [Middleton et al., 1990]; therefore this split is a reasonable compromise between the composition of the emissions and the photochemical production. Atkinson [1994] recommends the following rate constants for the reaction of $\mathrm{HO}$ with acetone and methylethyl ketone:

Acetone

$$
k=5.34 \times 10^{-18} \mathrm{~cm}^{3} \mathrm{~s}^{-1} \times T^{2} \exp (-230 / T)
$$

Methyl ethyl ketone

$$
k=3.24 \times 10^{-18} \mathrm{~cm}^{3} \mathrm{~s}^{-1} \times T^{2} \exp (414 / T)
$$

The rate constant of $\mathrm{HO}$ with KET was derived from the arithmetic means of the activation energies and the rate constants at $298 \mathrm{~K}$ (Table $2 \mathrm{c}$ ).

The scheme for the reactions of KET was based upon the following considerations. Acetone reacts to produce $\mathrm{CH}_{3} \mathrm{COCH}_{2} \mathrm{O} \cdot$ (reactions (39)-(40)). The $\mathrm{CH}_{3} \mathrm{COCH}_{2} \mathrm{O} \cdot$ may either react with $\mathrm{O}_{2}$ to form methylglyoxal and $\mathrm{HO}_{2}$ or to decompose to produce an acetyl peroxy radical and formaldehyde. The branching ratio between these two reaction processes is uncertain [Atkinson and Lloyd, 1984; Le Bras, 1997]. For the RACM mechanism, decomposition of the $\mathrm{CH}_{3} \mathrm{COCH}_{2} \mathrm{O}_{2} \cdot$ radical was not considered, and further experimental data are required:

$$
\begin{aligned}
& \mathrm{CH}_{3} \mathrm{COCH}_{3}+\mathrm{HO}\left(+\mathrm{O}_{2}\right) \rightarrow \mathrm{CH}_{3} \mathrm{COCH}_{2} \mathrm{O}_{2} \cdot+\mathrm{H}_{2} \mathrm{O} \\
& \mathrm{CH}_{3} \mathrm{COCH}_{2} \mathrm{O}_{2} \cdot+\mathrm{NO} \rightarrow \mathrm{CH}_{3} \mathrm{COCH}_{2} \mathrm{O} \cdot+\mathrm{NO}_{2} \\
& \mathrm{CH}_{3} \mathrm{COCH}_{2} \mathrm{O} \cdot+\mathrm{O}_{2} \rightarrow a \mathrm{CH}_{3} \mathrm{COCHO}+a \mathrm{HO}_{2} \\
& +b \mathrm{CH}_{3} \mathrm{CO}_{3} \cdot+b \text { HCHO }
\end{aligned}
$$

The reactions of methyl ethyl ketone are known to be considerably more complicated. The reaction of methyl ethyl ketone with $\mathrm{HO}$ and the subsequent reaction of the organic with $\mathrm{O}_{2}$ radical leads to [Atkinson, 1994]

$$
\begin{aligned}
\mathrm{CH}_{3} \mathrm{CH}_{2} \mathrm{COCH}_{3}+\mathrm{HO}\left(+\mathrm{O}_{2}\right) \rightarrow 0.46 \cdot \mathrm{O}_{2} \mathrm{CH}_{2} \mathrm{CH}_{2} \mathrm{C}(\mathrm{O}) \mathrm{CH}_{3} \\
+0.46 \mathrm{CH}_{3} \mathrm{CH}\left(\mathrm{O}_{2} \cdot\right) \mathrm{C}(\mathrm{O}) \mathrm{CH}_{3} \\
+0.08 \mathrm{CH}_{3} \mathrm{CH}_{2} \mathrm{C}(\mathrm{O}) \mathrm{CH}_{2} \mathrm{O}_{2} \cdot+\mathrm{H}_{2} \mathrm{O}
\end{aligned}
$$

The three peroxy radicals may react with $\mathrm{NO}$ or other species to produce oxy radicals.

$\mathrm{CH}_{3} \mathrm{C}(\mathrm{O}) \mathrm{CH}_{2} \mathrm{CH}_{2} \mathrm{O}_{2}+\mathrm{NO} \rightarrow \mathrm{CH}_{3} \mathrm{C}(\mathrm{O}) \mathrm{CII}_{2} \mathrm{CH}_{2} \mathrm{O}+\mathrm{NO}_{2}$

$$
\begin{aligned}
& \mathrm{CH}_{3} \mathrm{CH}\left(\mathrm{O}_{2} \cdot\right) \mathrm{C}(\mathrm{O}) \mathrm{CH}_{3}+\mathrm{NO} \\
& \quad \rightarrow \mathrm{CH}_{3} \mathrm{CH}(\mathrm{O} \cdot) \mathrm{C}(\mathrm{O}) \mathrm{CH}_{3}+\mathrm{NO}_{2} \\
& \mathrm{CH}_{3} \mathrm{CH}_{2} \mathrm{C}(\mathrm{O}) \mathrm{CH}_{2} \mathrm{O}_{2} \cdot+\mathrm{NO} \rightarrow \mathrm{CH}_{3} \mathrm{CH}_{2} \mathrm{C}(\mathrm{O}) \mathrm{CH}_{2} \mathrm{O}+\mathrm{NO}_{2}
\end{aligned}
$$

According to the rate constants given by Atkinson [1994], 58\% of the primary oxy radicals, $\mathrm{CH}_{3} \mathrm{C}(\mathrm{O}) \mathrm{CH}_{2} \mathrm{CH}_{2} \mathrm{O} \cdot$ and $\mathrm{CH}_{3} \mathrm{CH}_{2} \mathrm{C}(\mathrm{O}) \mathrm{CH}_{2} \mathrm{O}$, react through isomerization and the remaining $42 \%$ react through abstraction of $\mathrm{H}$ atoms by $\mathrm{O}_{2}$.

The isomerization reactions involve the transfer of an $\mathrm{H}$ atom to the terminal $\mathrm{O}$ atom:

$$
\begin{aligned}
& \mathrm{CH}_{3} \mathrm{C}(\mathrm{O}) \mathrm{CH}_{2} \mathrm{CH}_{2} \mathrm{O} \cdot \rightarrow \mathrm{HOCH}_{2} \mathrm{CH}_{2} \mathrm{C}(\mathrm{O}) \mathrm{CH}_{2} \cdot \\
& \mathrm{CH}_{3} \mathrm{CH}_{2} \mathrm{C}(\mathrm{O}) \mathrm{CH}_{2} \mathrm{O}^{\cdot} \rightarrow \mathrm{HOCH}_{2} \mathrm{C}(\mathrm{O}) \mathrm{CH}_{2} \mathrm{CH}_{2} .
\end{aligned}
$$

In the RACM mechanism the effects of the $\mathrm{HOCH}_{2} \mathrm{CH}_{2} \mathrm{C}(\mathrm{O}) \mathrm{CH}_{2} \cdot$ radical are represented through the reactions of $\mathrm{ALD}, \mathrm{XO}_{2}$, and $\mathrm{HO}_{2}$, and the effects of the $\mathrm{HOCH}_{2} \mathrm{C}(\mathrm{O}) \mathrm{CH}_{2} \mathrm{CH}_{2}$. radical are represented through the reactions of MGLY $; \mathrm{XO}_{2}$, and $\mathrm{HO}_{2}$.

The $\mathrm{H}$ abstraction reactions produce aldehydes and an $\mathrm{HO}_{2}$ :

$$
\mathrm{CH}_{3} \mathrm{C}(\mathrm{O}) \mathrm{CH}_{2} \mathrm{CH}_{2} \mathrm{O} \cdot+\mathrm{O}_{2} \rightarrow \mathrm{CH}_{3} \mathrm{C}(\mathrm{O}) \mathrm{CH}_{2} \mathrm{CHO}+\mathrm{HO}_{2}
$$

$$
\mathrm{CH}_{3} \mathrm{CH}_{2} \mathrm{C}(\mathrm{O}) \mathrm{CH}_{2} \mathrm{O} \cdot+\mathrm{O}_{2} \rightarrow \mathrm{CH}_{3} \mathrm{CH}_{2} \mathrm{C}(\mathrm{O}) \mathrm{CHO}+\mathrm{HO}_{2}
$$


$\mathrm{CH}_{3} \mathrm{C}(\mathrm{O}) \mathrm{CH}_{2} \mathrm{CHO}$ is treated as $\mathrm{ALD}$ and $\mathrm{CH}_{3} \mathrm{CH}_{2} \mathrm{C}(\mathrm{O}) \mathrm{CHO}$ is treated as MGLY.

The secondary oxy radical $\mathrm{CH}_{3} \mathrm{CH}(\mathrm{O} \cdot) \mathrm{C}(\mathrm{O}) \mathrm{CH}_{3}$ rapidly decomposes [Atkinson, 1994]:

$$
\mathrm{CH}_{3} \mathrm{CH}(\mathrm{O} \cdot) \mathrm{C}(\mathrm{O}) \mathrm{CH}_{3}\left(+\mathrm{O}_{2}\right) \rightarrow \mathrm{CH}_{3} \mathrm{CHO}+\mathrm{CH}_{3} \mathrm{C}(\mathrm{O}) \mathrm{O}_{2} \text {. }
$$

These products are characterized as ALD and $\mathrm{ACO}_{3}$ in the RACM mechanism.

For methyl ethyl ketone if (43)-(50) are considered along with the radical yields and given the assumption that KET represents a $50 \%-50 \%$ mixture of acetone and methyl ethyl ketone, (51) for the reaction of the KETP radical with NO is obtained:

$$
\begin{gathered}
\mathrm{KETP}+\mathrm{NO} \rightarrow 0.54 \mathrm{MGLY}+0.46 \mathrm{ALD}+0.23 \mathrm{ACO}_{3} \\
+0.77 \mathrm{HO}_{2}+0.16 \mathrm{XO}_{2}+\mathrm{NO}_{2}
\end{gathered}
$$

The dicarbonyl species glyoxal (GLY) and methylglyoxal (MGLY) have very similar chemistry and therefore their product yields were derived in a similar manner. For the reaction of glyoxal with HO, Atkinson [1994] recommends two reaction channels:

$$
\begin{array}{r}
\mathrm{HCOCOH}+\mathrm{HO} \rightarrow \mathrm{HCO}+\mathrm{CO}+\mathrm{H}_{2} \mathrm{O} \\
\mathrm{HCOCOH}+\mathrm{HO}\left(+\mathrm{O}_{2}\right) \rightarrow \mathrm{HC}(\mathrm{O}) \mathrm{C}(\mathrm{O}) \mathrm{O}_{2}+\mathrm{H}_{2} \mathrm{O}
\end{array}
$$

The relative percent yields of (52) and (53) are $60 \%$ and $40 \%$, respectively. The $\mathrm{HCO}$ radical reacts to produce $\mathrm{HO}_{2}$ and $\mathrm{CO}$ :

$$
\mathrm{HCO} \cdot+\mathrm{O}_{2} \rightarrow \mathrm{HO}_{2}+\mathrm{CO}
$$

If (52) and (54) were the only reaction pathways for HCO$\mathrm{COH}$, the overall reaction would be

$$
\mathrm{HCOCOH}+\mathrm{HO}\left(+\mathrm{O}_{2}\right) \rightarrow \mathrm{HO}_{2}+2 \mathrm{CO}+\mathrm{H}_{2} \mathrm{O}
$$

An explicit consideration of the reaction pathway for $\mathrm{HC}(\mathrm{O})$ $\mathrm{C}(\mathrm{O}) \mathrm{O}_{2} \cdot$ would require two additional species $\mathrm{HC}(\mathrm{O})$ $\mathrm{C}(\mathrm{O}) \mathrm{O}_{2} \cdot$ and $\mathrm{HC}(\mathrm{O}) \mathrm{C}(\mathrm{O}) \mathrm{O}_{2} \mathrm{NO}_{2}$ and many additional $\mathrm{RO}_{2} \cdot$ reactions. The subsequent chemistry of $\mathrm{HC}(\mathrm{O}) \mathrm{C}(\mathrm{O}) \mathrm{O}_{2} \cdot$ is highly uncertain, but it would be expected that the radical reacts with NO:

$$
\mathrm{HC}(\mathrm{O}) \mathrm{C}(\mathrm{O}) \mathrm{O}_{2} \cdot+\mathrm{NO}\left(+\mathrm{O}_{2}\right) \rightarrow \mathrm{HO}_{2}+\mathrm{CO}+\mathrm{CO}_{2}+\mathrm{NO}_{2}
$$

Probably, $\mathrm{HC}(\mathrm{O}) \mathrm{C}(\mathrm{O}) \mathrm{O}_{2} \cdot$ can react with $\mathrm{NO}_{2}$ to produce a peroxy nitrate with a thermal stability similar to peroxyacetyl nitrate (PAN).

$$
\mathrm{HC}(\mathrm{O}) \mathrm{C}(\mathrm{O}) \mathrm{O}_{2} \cdot+\mathrm{NO}_{2} \rightarrow \mathrm{HC}(\mathrm{O}) \mathrm{C}(\mathrm{O}) \mathrm{O}_{2} \mathrm{NO}_{2}
$$

Because of the $\alpha$-dicarbonyl group and the aldehyde hydrogen atom, $\mathrm{HC}(\mathrm{O}) \mathrm{C}(\mathrm{O}) \mathrm{O}_{2} \mathrm{NO}_{2}$ would be expected to have a much higher $\mathrm{HO}$ reaction rate constant and to have a much higher photolysis frequency than PAN:

$$
\mathrm{HC}(\mathrm{O}) \mathrm{C}(\mathrm{O}) \mathrm{O}_{2} \mathrm{NO}_{2}+\mathrm{HO} \rightarrow \mathrm{CO}+\mathrm{CO}_{2}+\mathrm{NO}_{3}+\mathrm{H}_{2} \mathrm{O}
$$

$$
\begin{array}{r}
\mathrm{HC}(\mathrm{O}) \mathrm{C}(\mathrm{O}) \mathrm{O}_{2} \mathrm{NO}_{2}+h \nu\left(+\mathrm{O}_{2}\right) \\
\rightarrow \mathrm{HO}_{2}+\mathrm{CO}+\mathrm{CO}_{2}+\mathrm{NO}_{3}
\end{array}
$$

Given these differences between $\mathrm{HC}(\mathrm{O}) \mathrm{C}(\mathrm{O}) \mathrm{O}_{2} \mathrm{NO}_{2}$ and PAN, it would be wrong to group this peroxynitrate with PAN. However, the mechanism can still be simplified if a few assumptions are made. The $\mathrm{NO}$ pathway for $\mathrm{HC}(\mathrm{O}) \mathrm{C}(\mathrm{O}) \mathrm{O}_{2}{ }^{\circ}$ involves two peroxy radicals $\mathrm{HC}(\mathrm{O}) \mathrm{C}(\mathrm{O}) \mathrm{O}_{2} \cdot$ and $\mathrm{HO}_{2}$ and therefore two possible $\mathrm{NO}$ to $\mathrm{NO}_{2}$ conversions. The $\mathrm{NO}_{2}$ pathway produces an overall total of fewer than $1 \mathrm{NO}$ to $\mathrm{NO}_{2}$ conversions because of $\mathrm{HC}(\mathrm{O}) \mathrm{C}(\mathrm{O}) \mathrm{O}_{2} \mathrm{NO}_{2}$ formation and consumption of the $\mathrm{HO}$ radical in (58). If it is assumed that the $\mathrm{NO}$ and $\mathrm{NO}_{2}$ pathways for $\mathrm{HC}(\mathrm{O}) \mathrm{C}(\mathrm{O}) \mathrm{O}_{2}$ are of equal importance, then the total number of $\mathrm{NO}$ to $\mathrm{NO}_{2}$ conversions will be close to 1 . This assumption is justificd because only the photolysis reaction directly produces additional peroxy radicals (although the rapid photolysis of $\mathrm{NO}_{3}$ does produce some $\mathrm{O}_{3}$ ). The $\mathrm{HC}(\mathrm{O}) \mathrm{C}(\mathrm{O}) \mathrm{O}_{2} \cdot$ reaction pathway will have about the same effect on $\mathrm{O}_{3}$ and $\mathrm{NO}_{x}$ concentrations as the pathway given by (55). For this reason and given their high uncertainty, the possible $\mathrm{HC}(\mathrm{O}) \mathrm{C}(\mathrm{O}) \mathrm{O}_{2}$ : reactions are ignored. Therefore the RACM model reaction is completely analogous to (55):

$$
\mathrm{GLY}+\mathrm{HO}\left(+\mathrm{O}_{2}\right) \rightarrow \mathrm{HO}_{2}+2 \mathrm{CO}+\mathrm{H}_{2} \mathrm{O}
$$

The major difference for MGLY is that its reaction with $\mathrm{HO}$ produces an acetyl radical rather than an $\mathrm{HO}_{2}$ (Table $2 \mathrm{~b}$ ). The reactions of DCB are described in section 3.4.3 because unsaturated dicarbonyls are products of aromatic oxidation.

The reactions of the $\mathrm{NO}_{3}$ radical with carbonyl species HCHO, ALD, GLY, MGLY; and DCB are included because these reactions can be significant during the nighttime [Stockwell and Calvert, 1983]. The $\mathrm{NO}_{3}$ radical reacts with saturated aldehydes through hydrogen atom abstraction:

$$
\mathrm{RCHO}+\mathrm{NO}_{3}\left(+\mathrm{O}_{2}\right) \rightarrow \mathrm{RCO}_{3} \cdot+\mathrm{HNO}_{3}
$$

The rate constants for the reaction of $\mathrm{NO}_{3}$ with $\mathrm{HCHO}$ and acetaldehyde (ALD) were taken from DeMore et al. [1994]. The $\mathrm{NO}_{3}-\mathrm{MGLY}$ rate constant was assumed to be equal to the rate constant of ALD. For glyoxal there are only aldehydic hydrogen atoms; therefore the rate constant for GLY was estimated as $2 \times k\left(\right.$ acetaldehyde $\left.+\mathrm{NO}_{3}\right)-k\left(\right.$ ethane $\left.+\mathrm{NO}_{3}\right)$. The activation energies for the reactions of $\mathrm{NO}_{3}$ radical with all these aldehydes were assumed to be equal to the activation energy for the acetaldehyde $+\mathrm{NO}_{3}$ reaction given by DeMore et al. [1994]. The rate constant for the $\mathrm{DCB}+\mathrm{NO}_{3}$ reaction was taken from the estimates of Bierbach et al. [1994], and the activation energy was estimated to be the mean of the activation energy for the reaction of $\mathrm{NO}_{3}$ with acetaldehyde and the activation energies for the reaction of $\mathrm{NO}_{3}$ with unbranched internal alkenes.

Photolysis of carbonyls represents a significant loss process for these species. The quantum yields, cross sections, along with representative photolysis frequencies used for the carbonyl species are given in Table 2a. The photolysis frequencies for $\mathrm{HCHO}$ and ALD remain nearly unchanged from those used by Stockwell et al. [1990], while the KET values increased by about $10 \%$. The photolysis frequencies for GLY is significantly changed due to new recommendations from Atkinson et al. [1992b]. For MGLY, new values for the cross sections [Atkinson et al., 1992b; Staffelbach et al., 1995] and the quantum yield measurements [Koch and Moortgat, 1996] were adopted.

\subsection{Alkenes}

Alkenes are important constituents of the polluted and rural troposphere. Owing to the double bond, they are very reactive 
Table 5. Estimated Rate Constants for Alkenes Used for All Alkenes for Which Data Were Not Available

\begin{tabular}{|c|c|c|c|c|c|c|c|}
\hline \multirow{2}{*}{$\begin{array}{c}\text { RACM } \\
\text { Mechanism } \\
\text { Species }\end{array}$} & \multirow[b]{2}{*}{$\begin{array}{l}\text { Alkene } \\
\text { Structure }\end{array}$} & \multicolumn{2}{|c|}{$\mathrm{HO}$} & \multicolumn{2}{|c|}{$\mathrm{NO}_{3}$} & \multicolumn{2}{|l|}{$\mathrm{O}_{3}$} \\
\hline & & $\begin{array}{c}k(298 \mathrm{~K}) \\
\mathrm{cm}^{3} \mathrm{~s}^{-1}\end{array}$ & 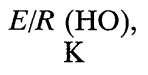 & $\begin{array}{c}k(298 \mathrm{~K}) \\
\mathrm{cm}^{3} \mathrm{~s}^{-1}\end{array}$ & $\begin{array}{c}E / R, \\
\mathrm{~K}\end{array}$ & $\begin{array}{c}k(298 \mathrm{~K}) \\
\mathrm{cm}^{3} \mathrm{~s}^{-1}\end{array}$ & $\begin{array}{c}E / R, \\
\mathrm{~K}\end{array}$ \\
\hline \multirow[t]{2}{*}{ OLT } & $\mathrm{RCH}=\mathrm{CH}_{2}$ & $3.2 \times 10^{-11}$ & -500 & $1.0 \times 10^{-14}$ & 1000 & $1.0 \times 10^{-17}$ & 1700 \\
\hline & $\mathrm{R}_{2} \mathrm{C}=\mathrm{CH}_{2}$ & $5.8 \times 10^{-11}$ & -500 & $4.5 \times 10^{-13}$ & 150 & $1.0 \times 10^{-17}$ & 1700 \\
\hline \multirow[t]{3}{*}{ OLI } & $\mathrm{RCH}=\mathrm{CHR}$ & $6.4 \times 10^{-11}$ & -500 & $4.5 \times 10^{-13}$ & 150 & $1.5 \times 10^{-16}$ & 1000 \\
\hline & $\mathrm{R}_{2} \mathrm{C}=\mathrm{CHR}$ & $8.8 \times 10^{-11}$ & -500 & $1.0 \times 10^{-11}$ & -500 & $5.0 \times 10^{-16}$ & 800 \\
\hline & $\mathrm{R}_{2} \mathrm{C}-\mathrm{CR}_{2}$ & $10.4 \times 10^{-11}$ & -500 & $5.7 \times 10^{-11}$ & -500 & $1.1 \times 10^{-15}$ & 300 \\
\hline
\end{tabular}

species that have relatively high rate constants for reaction with $\mathrm{HO}$ radical and they react with ozone and $\mathrm{NO}_{3}$ radicals [Finlayson-Pitts and Pitts, 1986]. Four model species were used to represent the anthropogenic emitted alkenes to better represent their diverse reactivities. Ethene is explicitly represented by ETE because of its lower rate constants for reactions with $\mathrm{HO}$ and $\mathrm{O}_{3}$ and its relatively high concentrations. Propene and other terminal alkenes (alkenes with a double bond attached to a carbon atom at an end of the molecule) are represented by OLT. Internal alkenes (alkenes with a double bond within the molecule) such trans-2-butene or cycloalkenes are represented by the model species OLI. Dienes and 1,3butadiene are aggregated into a new model species, DIEN. DIEN was introduced into the RACM mechanism because the rate constant for the reaction of 1,3-butadiene with $\mathrm{HO}$ is very different from the other terminal alkenes and its chemistry is more like isoprene than terminal alkenes [Atkinson, 1994].

For the RACM mechanism the rate constants for the reactions of alkenes with $\mathrm{HO}, \mathrm{NO}_{3}$, and $\mathrm{O}_{3}$ were taken from Atkinson [1994] when possible. For alkenes not listed by Atkinson [1994] the rate constants were estimated by taking the mean of alkenes with known rate constants and similar structure (Table 5).

3.5.1. Reaction with HO. The reaction of HO with alkenes occurs by addition to the double bond, followed by the addition of $\mathrm{O}_{2}$ to form a peroxy radical. The peroxy radicals may react with $\mathrm{NO}, \mathrm{NO}_{3}$, or $\mathrm{RO}_{2}$ to produce oxy radicals which are believed to decompose completely [Atkinson, 1994]. The only significant exception is the oxy radical resulting from ethene; about $20 \%$ of this reacts via $\mathrm{H}$ abstraction by $\mathrm{O}_{2}$ which produce hydroxyacetaldehyde $\left(\mathrm{H}_{2} \mathrm{C}(\mathrm{OH}) \mathrm{CHO}\right)$ which is treated as ALD in RACM:

$$
\mathrm{II}_{2} \mathrm{C}(\mathrm{OH}) \mathrm{CH}_{2} \mathrm{O} \cdot+\mathrm{O}_{2} \rightarrow \mathrm{H}_{2} \mathrm{C}(\mathrm{OH}) \mathrm{CHO}+\mathrm{HO}_{2}
$$

The rate constants for the reactions of the model species OLT and OLI with $\mathrm{HO}$ producing the peroxy radicals OLTP and OLIP were calculated according to (12). In contrast to the alkanes, for which measured data for many rate constants are available, only a few measurements exist for alkenes [Atkinson, 1994]. Where no experimental rate constants were available, estimated values from Table 5 were taken as $k_{i, \mathrm{HO}}$ in (14). The composition of the model peroxy radicals ETEP, OLTP, and OLIP was calculated by using the same procedure that was applied to the alkanes. For alkenes higher than ethene the reaction with $\mathrm{HO}$ is so fast on a daily basis that on the regional scale an initial amount of alkenes would be almost completely consumed. Therefore according to (16), no reactivity weighting is required.
3.5.2. Alkene $+\mathrm{NO}_{3}$ radical. The initial reaction of $\mathrm{NO}_{3}$ with alkenes is addition to the double bond. The adduct reacts with $\mathrm{O}_{2}$ to form a peroxy radical which was represented by the model species OLN in the original RADM2 mechanism [Stockwell et al., 1990]. The $\mathrm{NO}_{3}$-alkene-peroxy radicals may react with $\mathrm{NO}, \mathrm{NO}_{3}$, or $\mathrm{RO}_{2} \cdot$ to form oxy radicals. For the oxy radicals produced in this reaction, two fundamentally different reaction pathways are possible: decomposition (pathway $\mathrm{D}$ ) or formation of organic nitrates (pathway $N$ ). Recent experimental studies on the reaction of $\mathrm{NO}_{3}$ which alkenes have shown that the product yields for the two pathways depend strongly on the structure of the alkene from which the $\mathrm{NO}_{3}$-alkene-oxy radical was formed [Barnes et al., 1990; Hjorth et al., 1990; Skov et al., 1992; Tuazon et al., 1993]. Radicals formed from unbranched alkenes and dienes produce high yields of carbonyl nitrates (pathway $\mathrm{N}$ ), while the reactions of $\mathrm{NO}_{3}$ with branched monoalkenes have higher yields of carbonyls and $\mathrm{NO}_{2}$ (pathway $\mathrm{D}$ ) and relatively low yields of carbonyl nitrates. To distinguish between these two reaction pathways, the species OLN in the RADM2 mechanism was replaced by two model species for the $\mathrm{NO}_{3}$-alkene-peroxy radical, OLNN, which primarily produces nitrates (pathway N), and OLND, which primarily decomposes to produce carbonyls and $\mathrm{NO}_{2}$ (pathway D). It is assumed that the total yield of OLNN and OLND is unity.

Following the general procedure for building the RACM mechanism, the product yields $\alpha_{i, \mathrm{NO}_{3}}^{\mathrm{Nitrate}}$ for the model species OLNN and OLND from the reactions of the species ETE, OLI, and OLT with $\mathrm{NO}_{3}$ depend on the rate constants $k_{i, \mathrm{NO}}$ and nitrate product yields, $\beta_{i, \mathrm{NO}_{3}}^{\mathrm{Nitrate}}$, for the reaction of the chemical species with $\mathrm{NO}_{3}$ and the composition of alkene emissions [Middleton et al., 1990]. Our estimated nitrate yields, $\beta_{i, \mathrm{NO}_{3}}^{\mathrm{Nitra}}$, for alkenes are compiled in Table 6 , but there are relatively few experiments, and the agreement between them is not good [Barnes et al., 1990; Hjorth et al., 1990; Tuazon et al., 1993]:

$$
\begin{aligned}
& \mathrm{OLT}+\mathrm{NO}_{3} \rightarrow \alpha_{\mathrm{OLT}, \mathrm{NO}_{3}}^{\mathrm{OLNN}} \mathrm{OLNN}+\left(1-\alpha_{\mathrm{OLT}, \mathrm{NO}_{3}}^{\mathrm{OLNN}}\right) \text { OLND } \\
& \mathrm{OLI}+\mathrm{NO}_{3} \rightarrow \alpha_{\mathrm{OLI}, \mathrm{NO}_{3}}^{\mathrm{OINN}} \mathrm{OLNN}+\left(1-\alpha_{\mathrm{OLI}, \mathrm{NO}_{3}}^{\mathrm{OLNN}}\right) \mathrm{OLND}
\end{aligned}
$$

with

$$
\alpha_{m, \mathrm{NO}_{3}}^{\mathrm{OLNN}}=\frac{\sum_{i \in m} E_{i} k_{i, \mathrm{NO}_{3}} \beta_{i, \mathrm{NO}_{3}}^{\mathrm{Nitrate}}}{\sum_{i \in m} E_{i} k_{i, \mathrm{NO}_{3}}}
$$

where $m$ is equal to either OLI or OLT. The reactions of OLNN and OLND with NO and with other peroxy radicals were described by Kirchner and Stockwell [1996a]. 
Table 6. Estimated Yields of Nitrate $\left(\beta_{i, \mathrm{NO}_{3}}^{\mathrm{Nitrate}}\right)$ Carbonyls and $\mathrm{NO}_{2}$ From Nitrate Radical-Alkene Adduct Reactions

\begin{tabular}{lcll}
\hline $\begin{array}{c}\text { Alkene } \\
\text { Structure }\end{array}$ & $\begin{array}{c}\text { Estimated } \\
\beta_{i, \mathrm{NO}}^{\text {Nitrate }}\end{array}$ & \multicolumn{1}{c}{$\begin{array}{c}\text { Estimated Yield of } \\
\text { Carbonyls and } \mathrm{NO}_{2}\end{array}$} \\
\hline & & ETE & \\
$\mathrm{CH}_{2}=\mathrm{CH}_{2}$ & 0.80 & & $0.40 \mathrm{HCHO}$ \\
& & OLT & \\
$\mathrm{RCH}=\mathrm{CH}_{2}$ & 0.80 & & $0.20 \mathrm{HCHO}+0.20 \mathrm{ALD}$ \\
$\mathrm{R}_{2} \mathrm{C}=\mathrm{CH}_{2}$ & 0.20 & & $0.80 \mathrm{HCHO}+0.80 \mathrm{ALD}$ \\
& & OLI & \\
$\mathrm{RCH}=\mathrm{CHR}$ & 0.60 & & $0.80 \mathrm{ALD}$ \\
$\mathrm{R}_{2} \mathrm{C}=\mathrm{CHR}$ & 0.10 & & $0.90 \mathrm{ALD}+0.90 \mathrm{KET}$ \\
$\mathrm{R}_{2} \mathrm{C}=\mathrm{CR}_{2}$ & 0 & & $2.00 \mathrm{KET}$ \\
\hline
\end{tabular}

3.5.3. Ozonolysis of alkenes. Ozone reacts with alkenes to form ozonides which immediately decompose to yield excited Criegee intermediates [Finlayson-Pitts and Pitts, 1986] and carbonyl species, for example,

$$
\begin{aligned}
& \mathrm{CH}_{3} \mathrm{CH}=\mathrm{CH}_{2}+\mathrm{O}_{3} \rightarrow \mathrm{CH}_{3} \mathrm{CHO}+\left[\mathrm{CH}_{2} \mathrm{OO}\right]^{\neq} \\
& \mathrm{CH}_{3} \mathrm{CH}=\mathrm{CH}_{2}+\mathrm{O}_{3} \rightarrow \mathrm{HCHO}+\left[\mathrm{CH}_{3} \mathrm{CHOO}\right]^{\neq}
\end{aligned}
$$

The branching ratio for the formation of Criegee intermediates from $\mathrm{CH}_{3} \mathrm{CH}=\mathrm{CH}_{2}$ was investigated by Horie and Moortgat [1991] and was found to be $\left[\mathrm{CH}_{2} \mathrm{OO}\right] /\left[\mathrm{R}_{2} \mathrm{COO}\right]=0.38: 0.62$. To estimate these branching ratios for other alkenes, this ratio of 4:6 was used for all $\mathrm{R}-\mathrm{CH}=\mathrm{CH}_{2}$ and for $\mathrm{R}_{2} \mathrm{C}=\mathrm{CHR}$. The $\mathrm{R}_{2} \mathrm{C}=\mathrm{CH}_{2}$ branching ratio was calculated to be 0.4 to be consistent with the branching ratios for $\mathrm{R}-\mathrm{CH}=\mathrm{CH}_{2}$ and $\mathrm{R}_{2} \mathrm{C}=\mathrm{CHR}$ :

$$
\frac{\left[\mathrm{CH}_{2} \mathrm{OO}\right]^{\neq}}{\left[\mathrm{R}_{2} \mathrm{COO}\right]^{\neq}} \approx \frac{\left[\mathrm{CH}_{2} \mathrm{OO}\right]^{\neq}}{[\mathrm{RCHOO}]^{\ddagger}} \frac{\left[\mathrm{RCHOO}^{\neq}\right.}{\left[\mathrm{R}_{2} \mathrm{COO}\right]^{\neq}} \approx \frac{4 \times 4}{6 \times 6} \approx 0.4
$$

The excited Criegee intermediates are either stabilized or they decompose. Table 7 shows the products and yields of excited Criegee intermediates from Atkinson [1994] and the yields assumed in this work. Some $\mathrm{HO}$ is directly produced through the decomposition of the excited Criegee intermediates [Atkinson and Aschmann, 1993; Atkinson, 1994]. However, direct production of $\mathrm{HO}$ is not enough to account for the concentrations determined by Atkinson and Aschmann [1993] even if the upper limits given in Table 7 are used.

Table 7. Products and Yields of Criegee Intermediates Used in This Work

\begin{tabular}{clcc}
\hline \multicolumn{1}{c}{ Criegee } & \multicolumn{1}{c}{ Product } & Atkinson [1994] & This Work \\
\hline$\left[\mathrm{CH}_{2} \mathrm{OO}\right]^{*}$ & $\mathrm{CH}_{2} \mathrm{O}_{2}$ & 0.37 & 0.37 \\
& $\mathrm{CO}_{2}+\mathrm{H}_{2}$ & -0.13 & 0.13 \\
& $\mathrm{CO}_{2}+2 \mathrm{H}$ & $0.06-0.1$ & 0.07 \\
& $\mathrm{CO}+\mathrm{H}_{2} \mathrm{O}$ & $0.31-0.58$ & 0.31 \\
& $\mathrm{HCO}+\mathrm{HO}$ & $\sim 0.12$ & 0.12 \\
{$\left[\mathrm{CH}_{3} \mathrm{CHOO}\right]^{\neq}$} & $\mathrm{CH}_{3} \mathrm{CHO}_{2}$ & $0.15-0.42$ & 0.3 \\
& $\mathrm{CO}_{2}+\mathrm{CH}_{4}$ & $0.14-0.17$ & 0.15 \\
& $\mathrm{CO}_{2}+\mathrm{CH}_{3}+\mathrm{H}$ & $0.17-0.34$ & 0.2 \\
& $\mathrm{CO}+\mathrm{CH}_{3} \mathrm{OH}$ & $0-0.07$ & 0.03 \\
& $\mathrm{CO}+\mathrm{CH}_{3}+\mathrm{HO}$ & $0.16-0.30$ & 0.3 \\
& $\mathrm{HCO}+\mathrm{CH}_{3} \mathrm{O}$ & $0-0.07$ & 0.03 \\
{$\left[\left(\mathrm{CH}_{3}\right)_{2} \mathrm{COO}\right]^{\neq}$} & $(\mathrm{CH})_{2} \mathrm{CO}_{2}$ & 0.3 & 0.3 \\
& $\mathrm{CH}_{3} \mathrm{C}(\mathrm{O}) \mathrm{CH}_{2}+\mathrm{HO}$ & 0.7 & 0.7 \\
& & &
\end{tabular}

The dominant atmospheric loss process of the stabilized Criegee intermediates appears to be its reaction with $\mathrm{H}_{2} \mathrm{O}$ therefore these products are included in the new mechanism. Paulson et al. [1992b] proposed that stabilized Criegee intermediates may react with $\mathrm{H}_{2} \mathrm{O}$ to produce $\mathrm{HO}$ :

$$
\mathrm{R}_{2} \mathrm{COO}+\mathrm{H}_{2} \mathrm{O} \rightarrow \mathrm{R}_{2} \mathrm{CO}+2 \mathrm{HO}
$$

This reaction was used to fit the $\mathrm{HO}$ yields reported by Atkinson [1994]. The HO yields were fit by varying the fraction of Criegee intermediates which react by (69). The fraction of Criegee intermediates that react by (69) was estimated to be $0.0,0.4$ and 0.6 for $\mathrm{CH}_{2} \mathrm{OO}, \mathrm{RCHOO}$, and $\mathrm{R}_{2} \mathrm{COO}$. The resulting $\mathrm{HO}$ yields for alkenes of various structures are given in Table 8.

The reaction of stabilized Criegee intermediates with water may also produce hydrogen peroxide [Becker et al., 1993]:

$$
\mathrm{R}_{2} \mathrm{COO}+\mathrm{H}_{2} \mathrm{O} \rightarrow \mathrm{R}_{2} \mathrm{CO}+\mathrm{H}_{2} \mathrm{O}_{2}
$$

A $\mathrm{H}_{2} \mathrm{O}_{2}$ yield of $0.5 \%$ was determined by the measurements for $\mathrm{CH}_{2} \mathrm{OO}$ and for $\mathrm{R}_{2} \mathrm{COO}$ the estimated yield was about $13 \%$. Formic acid is also produced from the reaction of $\mathrm{R}_{2} \mathrm{COO}$ with water [Moortgat et al., 1997]. The results of Moortgat et al. [1997] suggest that the $\mathrm{HCOOH}$ and higher organic acids are formed through the rapid decomposition of organic hydroperoxides. Assuming the yield for RCHOO to be the geometric mean of the $\mathrm{CH}_{2} \mathrm{OO}$ and $\mathrm{R}_{2} \mathrm{COO}$ yields and allowing for the formation of $\mathrm{HO}$ and organic acids, the following reactions result:

$$
\begin{aligned}
& \mathrm{CH}_{2} \mathrm{OO}+\mathrm{H}_{2} \mathrm{O} \rightarrow 0.005 \mathrm{H}_{2} \mathrm{O}_{2} \\
& \quad+0.005 \mathrm{HCHO}+0.995 \mathrm{HCOOH} \\
& \mathrm{RCHOO}+\mathrm{H}_{2} \mathrm{O} \rightarrow 0.025 \mathrm{H}_{2} \mathrm{O}_{2} \\
& \quad+0.80 \mathrm{HO}+0.425 \mathrm{RCHO}+0.575 \mathrm{RCOOH} \\
& \quad \mathrm{R}_{2} \mathrm{COO}+\mathrm{H}_{2} \mathrm{O} \rightarrow 0.1 \mathrm{H}_{2} \mathrm{O}_{2}+1.2 \mathrm{HO} \\
& +0.7 \mathrm{RC}(\mathrm{O}) \mathrm{R}+0.3 \mathrm{RC}(\mathrm{O}) \mathrm{OR}
\end{aligned}
$$

Further reactions of the ester, $\mathrm{RC}(\mathrm{O}) \mathrm{OR}$, are ignored because it is relatively unreactive and only a relatively small amount is produced through gas-phase reactions. The alcohols which result from gas-phase chemistry are treated similarly.

The chemistry of the unsaturated dicarbonyl species (DCB) and the unsaturated peroxynitrate (TPAN) has been revised by including $\mathrm{HO}$ and $\mathrm{NO}_{3}$ addition reactions and ozonolysis. These reactions are described below because unsaturated carbonyls and peroxynitrates are products of aromatic decay.

Table 8. Estimated HO Yiclds Produced From the Ozonolysis of Alkenes

\begin{tabular}{lcc}
\hline Alkene Structure & $\begin{array}{c}\text { Experimental } \\
\text { [Atkinson, 1994] }\end{array}$ & $\begin{array}{c}\text { Fit } \\
\text { (This Work) }\end{array}$ \\
\hline $\mathrm{CH}_{2}=\mathrm{CH}_{2}$ & 0.12 & 0.12 \\
$\mathrm{CH}_{2}=\mathrm{CHR}$ & 0.37 & 0.37 \\
$\mathrm{CH}_{2}=\mathrm{CR}_{2}$ & 0.84 & 0.78 \\
$\mathrm{CHR}=\mathrm{CHR}$ & 0.58 & 0.54 \\
$\mathrm{CHR}=\mathrm{CR}_{2}$ & 0.91 & 0.85 \\
$\mathrm{CR}_{2}=\mathrm{CR}_{2}$ & 1.02 & 1.06 \\
\hline
\end{tabular}




\subsection{Aromatic Chemistry}

Aromatic compounds are oxidized in the atmosphere through reaction with $\mathrm{HO}$. When aromatics react with $\mathrm{HO}$ radicals, the radicals either add to the aromatic ring or react with a substituent group. However, the reaction of $\mathrm{HO}$ radicals with aromatic substituent groups is usually a much less important channel than $\mathrm{HO}$ addition to the aromatic ring. For typical aromatic compounds containing alkyl substituent groups the measured fraction of $\mathrm{HO}$ radicals reacting with a substituent group ranges between 0.04 and 0.12 [Atkinson, 1994]. It is assumed that an overall fraction of 0.10 of the $\mathrm{HO}$ radicals react by abstraction with the aromatic compounds treated in the RACM mechanism.

The HO radicals that react with alkyl substituent groups abstract hydrogen atoms. This hydrogen atom abstraction leads to the formation of peroxy radicals which may react with NO or other species to form oxy radicals. Subsequently, the oxy radicals react with oxygen to produce aromatics with aldehyde or ketone substituent groups. The $\mathrm{NO}$ to $\mathrm{NO}_{2}$ conversions affected by the oxy radicals are accounted for by the $\mathrm{XO}_{2}$ operator radical in the RACM mechanism. The product aromatics with aldehyde or ketone substituent groups have relatively small yields relative to the products formed from the addition of $\mathrm{HO}$ to the aromatic ring; therefore the subsequent reactions of these products are not included.

3.6.1. Treatment of cresol formation from aromatic-HO adducts. The aromatic-HO adduct reacts with $\mathrm{O}_{2}$ to either abstract an $\mathrm{H}$ atom to form a cresol or to add the $\mathrm{O}_{2}$ to form a peroxy radical. In the original RADM2 mechanism, large amounts of cresols were formed from aromatic photooxidation: $25 \%$ from toluene and $17 \%$ from xylene. There is much disagreement in the literature about the relative importance of cresol formation, but the most recent measurements suggest that the cresol yields have been significantly overestimated. For example, Atkinson et al. [1989, 1991, 1992a] found in the presence of $\mathrm{NO}_{x}$ that the yield of cresol from the oxidation of toluene was $25 \%$, while for ortho-xylene, meta-xylene, and para-xylene the yields of cresol were 16, 21, and 19\%, respectively. More recent cxpcriments provide yields that are $20-$ $40 \%$ lower. Furthermore, cresol formation decreases with decreasing $\mathrm{NO}_{x}$ concentrations [Atkinson and Aschmann, 1994]. In the absence of $\mathrm{NO}_{x}$ they measured a cresol yield of $12 \%$ from toluene. The 2,3-dimcthylphenol yield from o-xylene was 4 times lower in experiments without $\mathrm{NO}_{x}$ than in experiments with $\mathrm{NO}_{x}$, although it is possible that in these experiments other phenolic compounds may have been formed which were not measured.

Even lower yields of cresols were measured by Bierbach [1994]. Bierbach used the photolysis of $\mathrm{H}_{2} \mathrm{O}_{2}$ as the $\mathrm{HO}$ radical source for the oxidation of aromatics. He determined that the yield of cresols increascd with increasing initial concentrations of $\mathrm{NO}_{x}, \mathrm{H}_{2} \mathrm{O}_{2}$, and aromatics. A cresol yield less of than $1 \%$ was measured for toluene if the initial $\mathrm{H}_{2} \mathrm{O}_{2}$ concentration was low. For high $\mathrm{H}_{2} \mathrm{O}_{2}$ initial concentrations, Bierbach measured a cresol yield of $7 \%$ if $\mathrm{NO}_{x}$ was absent. Overall, these results suggest that much cresol is produced through the reactions of the aromatic- $\mathrm{HO}$ adduct with $\mathrm{NO}_{x}, \mathrm{H}_{2} \mathrm{O}_{2}$, and aromatics. Thus high yields of cresol from aromatic oxidation should not be expected for most typical atmospheric conditions but to allow for highly polluted conditions with high $\mathrm{NO}_{x}$ concentrations the yield of cresol formation is dependent on the $\mathrm{NO}_{x}$ concentration in the RACM mechanism.
Three different aromatic-HO adducts were added to the mechanism: ADDT (from toluene), ADDX (from xylene), and ADDC (from cresol). The measured rate constants for the reactions of the methylhydroxycyclohexadienyl radical with $\mathrm{O}_{2}$ and $\mathrm{NO}_{2}$ [Knispel et al., 1990] were used for these adducts. The reactions of $\mathrm{NO}_{x}, \mathrm{H}_{2} \mathrm{O}_{2}$, and aromatics with the aromatic-HO adduct increase cresol yields. The difference in the cresol yields measured by Bierbach [1994] and Atkinson et al. [1994] can be further rationalized if the aromatic-HO adduct reacts with ozone through

aromatic- $\mathrm{HO}$ adduct $+\mathrm{O}_{3} \rightarrow \mathrm{Cresol}+\mathrm{HO}+\mathrm{O}_{2}$

Given that the aromatic-HO adduct reacts with $\mathrm{O}_{2}, \mathrm{NO}_{x}$, $\mathrm{H}_{2} \mathrm{O}_{2}$, and aromatics, it would be very surprising if it did not react with ozone. In the toluene experiments of Atkinson and Aschmann [1994] the concentrations of $\mathrm{O}_{2}$ and $\mathrm{O}_{3}$ were about $\sim 5 \times 10^{18}$ and $\sim 5 \times 10^{12} \mathrm{~cm}^{-3}$, respectively. Using the concentrations of $\mathrm{O}_{2}$ and $\mathrm{O}_{3}$ and the difference between the measured cresol yields of Bierbach [1994] and Atkinson et al. [1994], the rate constant for (74) is calculated to be about $10^{5}$ times greater than the rate constant for the reaction of aromatic-HO adduct with $\mathrm{O}_{2}$. This implies that the rate constant for the toluene-HO adduct with $\mathrm{O}_{3}$ is about $5 \times 10^{-11} \mathrm{~cm}^{3} \mathrm{~s}^{-1}$ which is near the rate constant for the reaction of the aromatic-HO adduct with $\mathrm{NO}_{2}, \sim 3.6 \times 10^{-11} \mathrm{~cm}^{3} \mathrm{~s}^{-1}$ [Knispel et al., 1990]. Including (74) in the mechanism improves the agreement between simulated concentrations and environmental chamber data for the experiments containing toluene.

For o-xylene oxidation experiments that did not contain $\mathrm{NO}_{x}$, a 2,3-dimethylphenol yield of $2.4 \pm 0.9 \%$ was measured [1tkinson and Aschmann, 1994]. They measured a 2,3dimethylphenol to 3,4-dimethylphenol ratio of 3:2 in systems containing $\mathrm{NO}_{x}$ and o-xylene. If this same ratio applies to the systems not containing $\mathrm{NO}_{x}$, the overall cresol yield is $4.0 \pm$ $1.5 \%$. This result implies that the reaction of the HO-xylene adduct with $\mathrm{O}_{3}$ must be somewhat slower than for the reaction of the HO-toluene adduct. A value of $1 \times 10^{-11} \mathrm{~cm}^{3} \mathrm{~s}^{-1}$ was assigned to the rate constant for the reaction of aromatic-HO adduct with $\mathrm{O}_{3}$.

3.6.2. Further parameterization of Aromatic-HO adduct reactions. In the RACM mechanism the aromatic-HO adduct reacts with $\mathrm{O}_{2}$ through addition to produce a peroxy radical as was assumed in several previous mechanisms [Lurmann et al., 1986; Calvert and Madronich, 1987; Stockwell et al., 1990]. The existence of an aromatic peroxy radical and its subsequent reaction mechanism are both highly uncertain [Barnes et al., 1996]. However, the mechanism presented here is consistent with available laboratory and environmental chamber data. Many more experimental data are needed to understand the atmospheric oxidation of aromatic species. If the aromatic-HO adduct reacts with $\mathrm{O}_{2}$ through addition, the resulting peroxy radical could react through three different pathways (Figure 1):

In pathway I, the peroxy radical may react with $\mathrm{NO}, \mathrm{NO}_{3}$, or $\mathrm{R}^{\prime} \mathrm{O}_{2}$. to produce an $\mathrm{RO} \cdot$ radical. The $\mathrm{RO} \cdot$ radical would decompose to form a C6-dicarbonyl-diene (A) and $\mathrm{HO}_{2}$.

In pathway II, the oxygen molecule can form a bridge across the ring followed by addition of a second oxygen molecule. The resulting radical reacts to form an $\mathrm{RO}$ that decomposes to produce unsaturated dicarbonyls, glyoxal or methylglyoxal, and $\mathrm{HO}_{2}$ radicals; these are represented in the RACM mechanism as DCB, GI,Y or MGLY, and $\mathrm{HO}_{2}$, respectively. Some of the adducts may decay through this channel because $\mathrm{C} 4$ - 


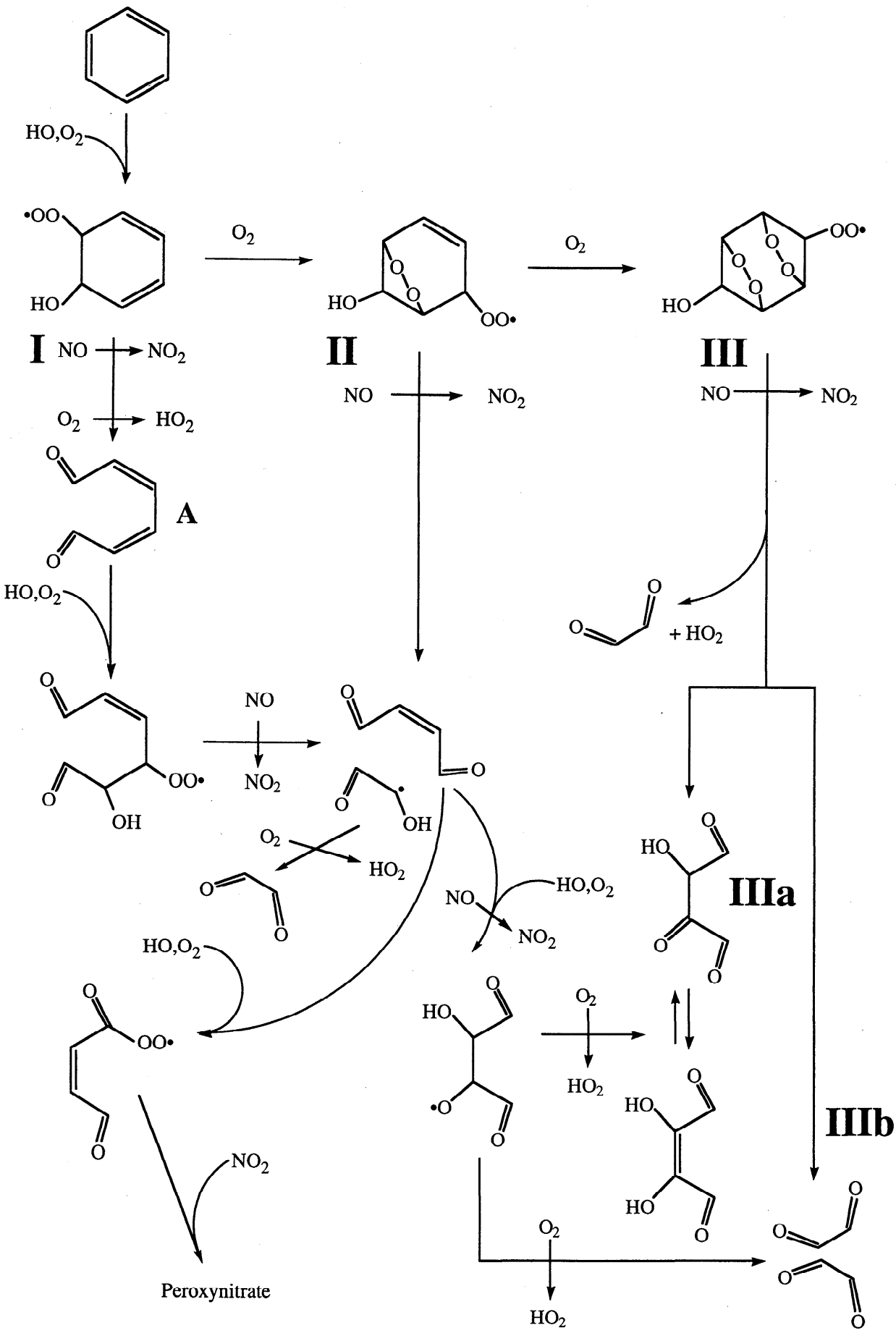

Figure 1. Possible decomposition pathways for the peroxy radical resulting from $\mathrm{O}_{2}$ addition to the aromatic-HO adduct.

dicarbonyls are experimentally measured aromatic compound decay products [Bierbach et al., 1994].

In pathway III, the peroxy radical can react to form a species with two $\mathrm{O}_{2}$ bridges across the ring followed by addition of a third oxygen molecule. This radical reacts to form an $\mathrm{RO}$. radical which decomposes to produce glyoxal or methylglyoxal and $\mathrm{HO}_{2}$ radicals.

The relative importance of these three possibilities for the reaction of the aromatic- $\mathrm{HO}-\mathrm{O}_{2}$ adduct was determined through the simulation of environmental chamber data including the experiments listed in Tables 9 and 10, but there is no guarantee that the environmental chamber simulations lead to a unique determination of their relative importance of the three reaction pathways. The calculation of the branching ratios is dependent in part upon the secondary products formed from the reactions of the unsaturated dicarbonyls. Knowledge of the chemistry of the unsaturated dicarbonyl products is very uncertain, and as new data become available, these branching ratios must be reevaluated.

The simulations show that the rate of ozone formation is different depending upon the decay channel (Figure 2). Channel I produces ozone at the greatest rate after a short inhibition 
Table 9. Comparison of RACM and RADM2 With SAPRC Ozone Data

\begin{tabular}{|c|c|c|c|c|c|c|c|c|c|c|c|}
\hline \multirow[b]{2}{*}{$\begin{array}{c}\text { SAPRC } \\
\text { Experi- } \\
\text { ment }\end{array}$} & \multirow[b]{2}{*}{$\begin{array}{c}\text { Principal } \\
\text { Organic } \\
\text { Compounds }^{\mathrm{a}}\end{array}$} & \multicolumn{5}{|c|}{ Peak Ozone Concentrations } & \multicolumn{5}{|c|}{ Time of Peak Ozone Concentrations } \\
\hline & & $\begin{array}{l}\text { Experi- } \\
\text { ment, } \\
\text { ppm }\end{array}$ & $\begin{array}{c}\text { RACM } \\
\text { Simulation, } \\
\text { ppm }\end{array}$ & $\begin{array}{l}\text { Differ- } \\
\text { ence, } \\
\%\end{array}$ & $\begin{array}{l}\text { RADM2 } \\
\text { Simulation, } \\
\text { ppm }\end{array}$ & $\begin{array}{l}\text { Differ- } \\
\text { ence, } \\
\%\end{array}$ & $\begin{array}{l}\text { Experi- } \\
\text { ment, } \\
\text { min }\end{array}$ & $\begin{array}{c}\text { RACM } \\
\text { Simulation, } \\
\text { min }\end{array}$ & $\begin{array}{l}\text { Differ- } \\
\text { ence, } \\
\%\end{array}$ & $\begin{array}{l}\text { RADM2 } \\
\text { Simulation, } \\
\text { min }\end{array}$ & $\begin{array}{l}\text { Differ- } \\
\text { ence, } \\
\%\end{array}$ \\
\hline EC-142 & ethene & 0.78 & 0.53 & -32.1 & 0.40 & -48.7 & $>360$ & $>360$ & $\ldots$ & $>360$ & . \\
\hline EC-143 & ethene & 1.09 & 0.83 & -23.9 & 0.79 & -27.5 & 212 & 290 & 36.8 & 350 & 65.1 \\
\hline EC-178 & $n$-butane & 0.37 & 0.47 & 27.0 & 0.46 & 24.3 & $>495$ & $>495$ & $\cdots$ & $>495$ & $\cdots$ \\
\hline EC-216 & propene & 0.56 & 0.73 & 30.4 & 0.62 & 10.7 & 465 & 525 & 12.9 & 520 & 11.8 \\
\hline EC-231 & mixture & 0.62 & 0.74 & 19.4 & 0.73 & 17.7 & 242 & 235 & -2.9 & 265 & 9.5 \\
\hline $\mathrm{EC}-232$ & mixture & 0.34 & 0.40 & 17.6 & 0.33 & -2.9 & $>390$ & $>390$ & $\ldots$ & $>390$ & $\ldots$ \\
\hline EC-233 & mixture & 0.33 & 0.44 & 33.3 & 0.42 & 25.8 & 262 & $>360$ & $\ldots$ & 340 & 29.8 \\
\hline EC-237 & mixture & 0.66 & 0.72 & 9.1 & 0.69 & 4.5 & 242 & 270 & 11.6 & 305 & 26.0 \\
\hline EC-238 & mixture & 0.69 & 0.82 & 18.8 & 0.79 & 14.5 & 435 & 450 & 3.4 & 510 & 17.2 \\
\hline EC-241 & mixture & 0.41 & 0.48 & 17.1 & 0.39 & -4.9 & $>360$ & $>360$ & $\cdots$ & $>360$ & $\cdots$ \\
\hline EC-242 & mixture & 0.68 & 0.73 & 7.9 & 0.69 & 0.9 & 105 & 111 & 5.7 & 123 & 17.1 \\
\hline EC-243 & mixture & 0.72 & 0.75 & 4.4 & 0.71 & -0.8 & 132 & 140 & 6.1 & 170 & 28.8 \\
\hline EC-245 & mixture & 0.89 & 0.92 & 3.4 & 0.88 & -1.0 & 175 & 192 & 9.7 & 220 & 25.7 \\
\hline EC-246 & mixture & 0.57 & 0.55 & -3.5 & 0.54 & -5.3 & $>575$ & $>570$ & $\ldots$ & $>570$ & $\ldots$ \\
\hline EC-254 & acetaldehyde & 0.26 & 0.31 & 19.2 & 0.23 & -11.5 & $>370$ & $>365$ & $\ldots$ & $>365$ & $\ldots$ \\
\hline EC-305 & $n$-butane & 0.4 & 0.54 & 35.0 & 0.55 & 37.5 & $>360$ & $>360$ & $\ldots$ & $>360$ & $\cdots$ \\
\hline EC-331 & $\begin{array}{l}\text { toluene }+ \\
n \text {-butane }\end{array}$ & 0.51 & 0.67 & 31.4 & 0.52 & 2.0 & 125 & 175 & 40.0 & 132 & 5.6 \\
\hline EC-340 & toluene & 0.41 & 0.40 & -2.4 & 0.35 & -14.6 & $>330$ & $>330$ & $\therefore$ & $>330$ & $\cdots$ \\
\hline EC-344 & $m$-xylene & 0.59 & 0.72 & 22.0 & 0.59 & 0.0 & 172 & 300 & 74.4 & 240 & 39.5 \\
\hline EC-345 & $m$-xylene & 0.4 & 0.50 & 25.0 . & 0.38 & -6.3 & 70 & 160 & 128.6 & 105 & 50.0 \\
\hline
\end{tabular}

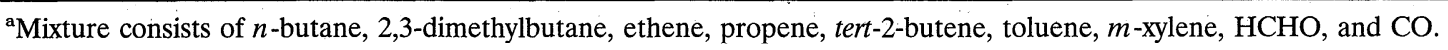

period. Channel II shows no inhibition period, but the rate of ozone production is somewhat less. Channel III shows the slowest rate of ozone formation, and the rate depends on whether the final products are an unsaturated-dihydroxydione, glyoxal and methyglyoxal (IIIa), or only glyoxal and methyglyoxal (IIIb). For xylene the agreement between simulations and experiment was best if $100 \%$ of the adducts react by channel II, while for toluene the best fit was obtained if it was assumed that $50 \%$ of the adducts react by channel II, $50 \%$ react by channel IIIb, and none react through channel I. It was furthermore assumed that the adducts have a $5 \%$ nitrate yield.

3.6.3. Treatment of unsaturated dicarbonyl products. The description of the chemistry of unsaturated dicarbonyl products (DCB) is primarily based on the work of Bierbach et al. [1994] and Wiesen et al. [1995]. For the reaction of HO with unsaturated dicarbonyls, Bierbach et al. found that $50 \%$ of the $\mathrm{HO}$ radicals react by $\mathrm{H}$ abstraction and about $50 \%$ add to the double bond. This ratio of abstraction to addition for the

Table 10. Comparison of RACM and RADM 2 With SAPRC Nitrogen Dioxide Data

\begin{tabular}{|c|c|c|c|c|c|c|c|c|c|c|c|}
\hline \multirow[b]{2}{*}{$\begin{array}{c}\text { SAPRC } \\
\text { Experi- } \\
\text { ment }\end{array}$} & \multirow[b]{2}{*}{$\begin{array}{c}\text { Principal } \\
\text { Organic } \\
\text { Compounds }^{\mathrm{a}}\end{array}$} & \multicolumn{5}{|c|}{ Peak $\mathrm{NO}_{2}$ Concentrations } & \multicolumn{5}{|c|}{ Time of Peak $\mathrm{NO}_{2}$ Concentrations } \\
\hline & & $\begin{array}{l}\text { Experi- } \\
\text { ment, } \\
\text { ppm }\end{array}$ & $\begin{array}{c}\text { RACM } \\
\text { Simulation, } \\
\text { ppm }\end{array}$ & $\begin{array}{l}\text { Differ- } \\
\text { ence, } \\
\%\end{array}$ & $\begin{array}{c}\text { RADM2 } \\
\text { Simulation, } \\
\text { ppm }\end{array}$ & $\begin{array}{c}\text { Differ- } \\
\text { ence, } \\
\%\end{array}$ & $\begin{array}{l}\text { Experi- } \\
\text { ment, } \\
\text { min }\end{array}$ & $\begin{array}{c}\text { RACM } \\
\text { Simulation, } \\
\text { min }\end{array}$ & $\begin{array}{c}\text { Differ- } \\
\text { ence, } \\
\%\end{array}$ & $\begin{array}{c}\text { RADM2 } \\
\text { Simulation, } \\
\text { min }\end{array}$ & $\begin{array}{c}\text { Differ- } \\
\text { ence, } \\
\%\end{array}$ \\
\hline EC-142 & ethene & 0.30 & 0.36 & 20.0 & 0.35 & 16.7 & 105 & 150 & 42.9 & 165 & 57.1 \\
\hline EC-143 & ethene & 0.38 & 0.41 & 7.4 & 0.41 & 6.6 & 60 & 93 & 55.0 & 105 & 75.0 \\
\hline EC-178 & $n$-butane & 0.07 & 0.08 & 17.1 & 0.08 & 17.1 & 75 & 110 & 46.7 & 100 & 33.3 \\
\hline EC-216 & propene & 0.37 & 0.39 & 5.4 & 0.38 & 4.1 & 105 & 80 & -23.8 & 90 & -14.3 \\
\hline EC-231 & mixture & 0.36 & 0.40 & 11.1 & 0.40 & 11.1 & 80 & 57 & -28.8 & 70 & -12.5 \\
\hline EC-232 & mixture & 0.34 & 0.36 & 5.9 & 0.36 & 5.3 & 165 & 180 & 9.1 & 195 & 18.2 \\
\hline EC-233 & mixture & 0.07 & 0.09 & 22.9 & 0.09 & 24.3 & 35 & 32 & -8.6 & 40 & 14.3 \\
\hline EC-237 & mixture & 0.37 & 0.39 & 5.7 & 0.39 & 5.1 & 65 & 58 & -10.8 & 72 & 10.8 \\
\hline EC-238 & mixture & 0.66 & 0.71 & 6.8 & 0.70 & 5.3 & 125 & 130 & 4.0 & 145 & 16.0 \\
\hline EC-241 & mixture & 0.35 & 0.36 & 3.4 & 0.36 & 2.3 & 140 & 130 & -7.1 & 140 & 0.0 \\
\hline EC-242 & mixture & 0.40 & 0.43 & 6.3 & 0.43 & 7.5 & 25 & 22 & -12.0 & 28 & 12.0 \\
\hline EC-243 & mixture & 0.40 & 0.42 & 5.0 & 0.42 & 5.0 & 35 & 27 & -22.9 & 39 & 11.4 \\
\hline EC-245 & mixture & 0.76 & 0.81 & 6.6 & 0.81 & 6.6 & 55 & 53 & -3.6 & 60 & 9.1 \\
\hline EC-246 & mixture & 0.37 & 0.37 & -0.5 & 0.37 & -1.1 & 148 & 170 & 14.9 & 190 & 28.4 \\
\hline EC-254 & acetaldehyde & 0.07 & 0.09 & 25.7 & 0.08 & 20.0 & 55 & 75 & 36.4 & 90 & 63.6 \\
\hline EC-305 & $n$-butane & 0.08 & 0.08 & 5.6 & 0.08 & 6.3 & 65 & 73 & 12.3 & 67 & 3.1 \\
\hline EC-331 & $\begin{array}{l}\text { toluene }+ \\
n \text {-butane }\end{array}$ & 0.33 & 0.36 & 7.6 & 0.36 & 7.6 & 45 & 45 & 0.0 & 40 & -11.1 \\
\hline EC-340 & toluene & 0.25 & 0.29 & 15.2 & 0.28 & 14.0 & 112 & 122 & 8.9 & 125 & 11.6 \\
\hline EC-344 & $m$-xylene & 0.45 & 0.48 & 6.7 & 0.49 & 7.8 & 45 & 60 & 33.3 & 55 & 22.2 \\
\hline EC-345 & $m$-xylene & 0.19 & 0.21 & 10.5 & 0.22 & 13.2 & 25 & 26 & 4.0 & 31 & 24.0 \\
\hline
\end{tabular}

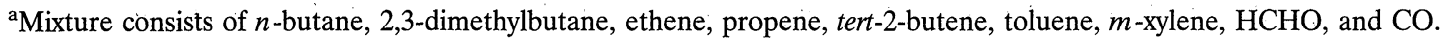




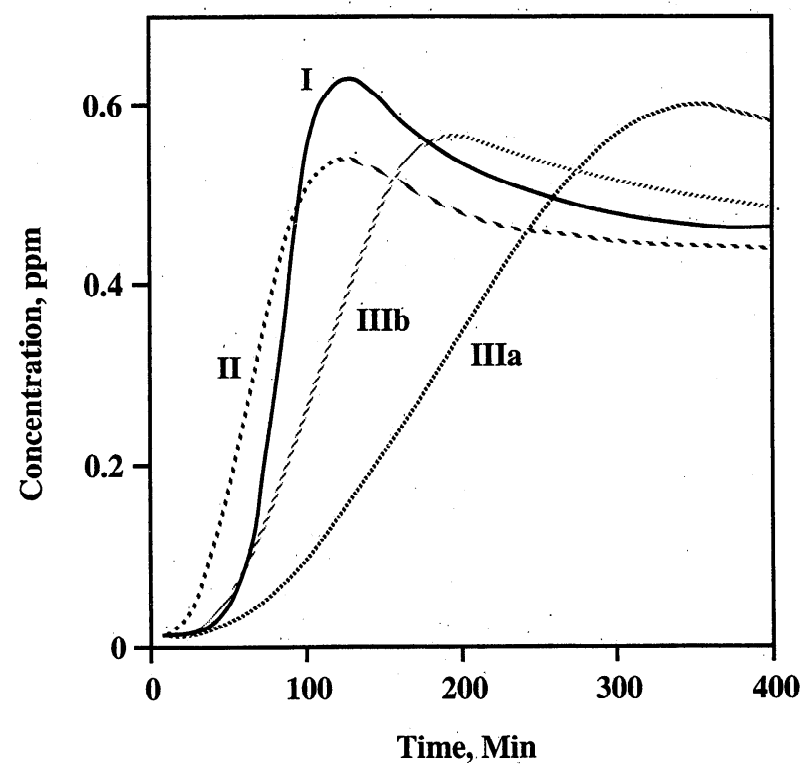

Figure 2. Simulated ozone formation profiles for decomposition pathways (I, II, and III) for the peroxy radical resulting from $\mathrm{O}_{2}$ addition to the aromatic-HO adduct aromatic decay (see text). Two different alternatives are presented for pathway III: IIIa, decomposition to 3,4-dihydroxy-3-hexene-2,5-dione, glyoxal and methyglyoxal; and IIIb, glyoxal and methyglyoxal only. These simulations are based upon EC-331.

reaction of $\mathrm{HO}$ with unsaturated dicarbonyls is similar to the ratio for the reaction of $\mathrm{HO}$ with methacrolein [Atkinson, 1994].

The products for the addition of $\mathrm{HO}$ with unsaturated dicarbonyls are based upon the several studies [Bierbach et al., 1994; Wiesen et al., 1995; Tuazon et al., 1985]. These researchers have studied the products of the $\mathrm{HO}$ addition reaction with 3-hexene-2,5-dione. The initial reaction sequence is the same as for alkenes: the $\mathrm{HO}$ radical adds to the double bond, and this is followed by addition of $\mathrm{O}_{2}$. The peroxy radical reacts with $\mathrm{NO}, \mathrm{NO}_{3}$, or other peroxy radicals to form an $\mathrm{RO}$ - radical. In contrast to most alkenes the $\mathrm{RO} \cdot$ decomposition reaction is much less important. About $30 \%$ of the $\mathrm{RO}$ radicals decompose, and $70 \%$ react via $\mathrm{H}$ abstraction by $\mathrm{O}_{2}$ or through $\mathrm{H}$ atom shift isomerization [Bierbach et al., 1994]. The main product for the reaction of $\mathrm{HO}$ with 3-hexene-2,5-dione was found to be 3,4-dihydroxy-3-hexene-2,5-dione, an unsaturateddihydroxy-dicarbonyl (UDD) [Wiesen, 1995]. To keep the RACM mechanism reasonably condensed, the peroxy radical is not treated separately rather it is represented by $\mathrm{XO}_{2}$ and its products from the peroxy radical-NO reaction.

The addition reaction of $\mathrm{HO}$ with 3,4-dihydroxy-3-hexene2,5-dione is very fast $\left(k \approx 2.7 \times 10^{-10} \mathrm{~cm}^{3} \mathrm{~s}^{-1}\right.$ ) [Wiesen, 1995]. Therefore it was assumed that the $\mathrm{H}$ abstraction reaction is unimportant for UDD. The main products of the 3,4dihydroxy-3-hexene-2,5-dione $+\mathrm{HO}$ reaction were $\mathrm{HO}_{2}$ and 3,3-dihydroxyhexane-2,4,5-trione: The 3,3-dihydroxyhexane2,4,5-trione may dehydrate to form hexane-2,3,4,5-tetraone. Generalizing this to UDD, the reaction products of $\mathrm{HO}$ with UDD are treated as $\mathrm{HO}_{2}$ and a mixture of ALD and KET.

The rate constant for the reaction of ozone with unsaturated dicarbonyl species (DCB) was based on the estimates of Bierbach et al: [1994]. There are no available product studies for the ozonolysis for these compounds. A Criegee mecha- nism was assumed, and the excited Criegee intermediates from the unsaturated dicarbonyls were characterized as $[\mathrm{HC}(\mathrm{O}) \mathrm{CHOO}]^{\neq},\left[\mathrm{HC}(\mathrm{O}) \mathrm{C}\left(\mathrm{CH}_{3}\right) \mathrm{OO}\right]^{\neq}$, and $[\mathrm{RC}(\mathrm{O}) \mathrm{CHOO}]^{\neq}$. $[\mathrm{HC}(\mathrm{O}) \mathrm{C}(\mathrm{R}) \mathrm{OO}]^{\neq}$is also a product of methacrolein ozonolysis [Kirchner and Stockwell, 1996b]. These excited Criegee intermediates would be expected to produce glyoxals, aldehydes, organic acids, and radicals. If it is further assumed that the ozonolysis of DCB produces equal yields of all three Criegee intermediates, and if the carbonyl products are included, the following reaction results:

$$
\begin{aligned}
\mathrm{DCB} & +\mathrm{O}_{3} \rightarrow 0.5 \mathrm{GLY}+0.62 \mathrm{MGLY}+0.16 \mathrm{ALD} \\
& +0.66 \mathrm{CO}+0.11 \mathrm{PAA}+0.11 \mathrm{ORA} 1+0.21 \mathrm{ORA} 2 \\
& +0.28 \mathrm{ACO}_{3}+0.29 \mathrm{HO}_{2}+0.21 \mathrm{HO}
\end{aligned}
$$

Unsaturated acyl peroxy radicals $\left(\mathrm{TCO}_{3}\right)$ are produced from DCB through photolysis and through reactions with $\mathrm{HO}$ and $\mathrm{NO}_{3}$ [Stockwell et al., 1990]. The reaction of unsaturated acyl peroxy radicals with NO leads to the production of $\mathrm{NO}_{2}, \mathrm{CO}_{2}$, and compounds of the form $\mathrm{R}-\mathrm{C}(\mathrm{O})-\mathrm{CH}=\mathrm{CH}$. $\mathrm{R}-\mathrm{C}(\mathrm{O})-\mathrm{CH}=\mathrm{CH} \cdot$ reacts with $\mathrm{O}_{2}$ to produce $\mathrm{R}-\mathrm{C}(\mathrm{O}) \mathrm{CHO}$, $\mathrm{CO}$, and $\mathrm{HO}_{2}$. The dicarbony compounds $\mathrm{R}-\mathrm{C}(\mathrm{O}) \mathrm{CHO}$ react with $\mathrm{HO}$ to produce acyl radicals and $\mathrm{CO}$ and $\mathrm{HO}_{2}$ radicals. These products are almost the same as the products from the aldehyde-hydrogen atom abstraction from methacrolein [Tuazon and Atkinson, 1990]:

$$
\begin{aligned}
& \mathrm{CH}_{2} \mathrm{CCH}_{3} \mathrm{CHO}+\mathrm{HO}\left(+\mathrm{O}_{2}\right) \\
& \rightarrow \mathrm{CH}_{2} \mathrm{C}\left(\mathrm{CH}_{3}\right) \mathrm{C}(\mathrm{O}) \mathrm{OO} \cdot+\mathrm{H}_{2} \mathrm{O} \\
& \mathrm{CH}_{2} \mathrm{C}\left(\mathrm{CH}_{3}\right) \mathrm{C}(\mathrm{O}) \mathrm{OO}+\mathrm{NO} \rightarrow \mathrm{NO}_{2}+\mathrm{CH}_{2} \mathrm{C}\left(\mathrm{CH}_{3}\right) \mathrm{C}(\mathrm{O}) \mathrm{O} \\
& \mathrm{CH}_{2} \mathrm{C}\left(\mathrm{CH}_{3}\right) \mathrm{C}(\mathrm{O}) \mathrm{O} \cdot \rightarrow \mathrm{CH}_{2} \mathrm{C}\left(\mathrm{CH}_{3}\right)+\mathrm{CO}_{2} \\
& \mathrm{CH}_{2} \mathrm{C}\left(\mathrm{CH}_{3}\right) \cdot\left(+2 \mathrm{O}_{2}\right) \rightarrow \mathrm{CH}_{3} \mathrm{C}(\mathrm{O}) \mathrm{O}_{2} \cdot+\mathrm{HCHO}^{77} \\
& \mathrm{HCHO}+\mathrm{HO}\left(+\mathrm{O}_{2}\right) \rightarrow \mathrm{CO}+\mathrm{HO}_{2}+\mathrm{H}_{2} \mathrm{O}
\end{aligned}
$$

To simplify the mechanism, the model species $\mathrm{TCO}_{3}$ was also used to represent the unsaturated acyl products. It is probable that the acyl radicals produced from unsaturated dicarbonyls react with $\mathrm{NO}_{2}$ to produce peroxynitrates; however, these products have not been observed. The comparable product, produced in methacrolein degradation, methacrylic peroxynitrate, has been reasonably well investigated. The rate constants and products for the reactions of our model species TPAN with $\mathrm{HO}$ and $\mathrm{O}_{3}$ are based upon the experimental measurements for methacrylic peroxynitrate. These reactions are further discussed by Kirchner and Stockwell [1996b].

3.6.4. Treatment of cresols. For the reaction of $\mathrm{HO}$ with cresol (CSL) it was assumed that an unsaturated dicarbonyl enol was formed which can isomerize via keto-enol-tautomerie into a saturated tricarbonyl compound which is treated as MGLY. For the reaction of $\mathrm{NO}_{3}$ with cresol it was assumed that the only reaction channel was the abstraction of the hydrogen atom from the $\mathrm{HO}$ group. There may be other possible reactions [Atkinson et al., 1994], but now no other reactions are known. The resulting phenoxy radical (PHO) can react with $\mathrm{NO}_{2}$ followed by a hydrogen shift to form ortho-nitrophenol: 
<smiles>Cc1ccc(O)c(C)c1</smiles><smiles>Cc1ccc([O-])c(C)c1</smiles>

The rate constant for (82) is not known; therefore the rate constant for the analogous $\mathrm{CH}_{3} \mathrm{O} \cdot+\mathrm{NO}_{2}$ addition reaction was used. For the $\mathrm{PHO}+\mathrm{HO}_{2}$ reaction the rate constant for $\mathrm{Ph}-\mathrm{CH}_{2} \mathrm{OO}+\mathrm{HO}_{2}$ was used. Because orthonitrophenol is less reactive than phenol by 1 order of magnitude, the products are described in the mechanism as 0.1CSL + ONIT. There is also experimental evidence for the formation of quinones from cresols [Wiesen et al., 1995] through the reactions of phenoxy radicals, but not enough is known about the quinone chemistry to include it in the RACM mechanism.

\subsection{Biogenic Organic Species}

The oxidation mechanism for isoprene was completely revised from the mechanism of Stockwell et al. [1990]. Isoprene is oxidized in the atmosphere through its reactions with $\mathrm{HO}$, $\mathrm{NO}_{3}$, and $\mathrm{O}_{3}$. Our oxidation scheme for the isoprene $+\mathrm{HO}$ reaction follows the measurements of Paulson et al. [1992a]. The new isoprene mechanism is a good representation of the atmospheric chemistry of methacrolein and MPAN. It includes treatment of isoprene ozonolysis, hydroperoxide production, and production of carbonitrates from the reaction of isoprene with $\mathrm{NO}_{3}$.

The RACM mechanism also includes a mechanism for the oxidation of $\alpha$-pinene and d-limonene. The new oxidation mechanism for $\alpha$-pinene and d-limonene is based upon the limited available experimental data for terpene reactions [Atkinson, 1994]. Because many of the reactions for $\alpha$-pinene and d-limonene oxidation are unknown, our mechanism was completed by using reactions that were analogous to known reactions for lower alkenes. To include the large number of different monoterpenes in the mechanism, it was necessary to group the individual monoterpenes into a few groups. This grouping was done according to similarities in rate constants and structure. Structure and rate constants of sabinene and $\Delta^{3}$-carene are similar to those of $\alpha$-pinene; therefore those terpenes are treated as $\alpha$-pinene. The mechanism was successfully tested against environmental chamber runs [Kirchner and Stockwell, 1996b].

\subsection{Parameters for Organic Peroxy Radical-Peroxy Radical Reactions and the Reactions of Nitrate Radical With Organic Peroxy Radicals}

The reactions of organic peroxy radical with $\mathrm{NO}, \mathrm{NO}_{3}, \mathrm{HO}_{2}$, and with other organic peroxy radicals were revised as described by Kirchner and Stockwell [1996a]. The revised rate constants were significantly different from those used by Stockwell et al. [1990] in several cases. Simulations made using the original mechanism and a version with the updated rate con- stants for the peroxy radical reactions were compared. The calculated concentrations (especially the nighttime concentrations) of PAN, higher organic hydroperoxides, peroxyacetic acid organic peroxy radicals, $\mathrm{HO}_{2}, \mathrm{HO}$, and $\mathrm{NO}_{3}$ were strongly affected by the revisions to peroxy radical chemistry. On the basis of that study it appcars that $\mathrm{RO}_{2} \cdot+\mathrm{NO}_{3}$ reactions are more important in the nighttime atmosphere than $\mathrm{RO}_{2}{ }^{+}$ $\mathrm{RO}_{2} \cdot$ reactions.

\section{Comparison With Environmental Chamber Data}

Simulation of environmental chamber experiments allows an atmospheric chemical mechanism to be tested as a complete system. The chamber typically contains a mixture of $\mathrm{NO}_{x}$ and organic species that are exposed to either sunlight or artificial light and concentration measurements are made as the compounds react [Carter et al., 1995]. Unfortunately, the usefulness of environmental chamber data is limited by several factors including the relatively high concentrations used, wall effects, and photolysis ratc uncertainties [Stockwell et al., 1990]. Chamber experiments are usually conducted at concentrations greater than those in ambient air to permit the concentrations to be measured. The walls of the chamber affect the concentrations of $\mathrm{O}_{3}, \mathrm{NO}_{x}$, aldehydes, and ketones and arc a significant source of radicals [Lurmann et al., 1986; Stockwell et al., 1990; Carter et al., 1995]. There may be differences between sunlight and the spectral distribution in the actinic flux in indoor chambers, while for outdoor chambers the photolysis rates may be uncertain due to clouds, wall adsorption and reflection, and other chamber effects [National Center for Atmospheric Research, (NCAR), 1986]. Taken together, all of these uncertainties in environmental chamber data limit the evaluation of predicted ozone concentrations to no better than about $\pm 30 \%$ [Stockwell et al., 1990].

The environmental chamber runs modeled here are the same experiments used to test both the mechanism of Lurmann et al. [1986] and the RADM2 mechanism [Stockwell et al., 1990]. Extremely extensive testing of the RADM2 mechanism that used the results from several different chambers showed that the experiments used here are representative for ozone, nitrogen oxides, and VOC [Carter and Lurmann, 1989]. The reevaluated data set of Carter et al. [1995] was used for these tests. The 20 environmental chamber runs were made using the Evacuable Chamber (EC) of the Statewide Air Pollution Research Center (SAPRC) of the University of California, Riverside [Carter et al., 1995]. This indoor chamber has a volume of $5800 \mathrm{~L}$. Typically, the relative humidity was near $50 \%$ for the modeled experiments. The RACM and RADM2 mechanisms were compared by running both against these experiments. The RADM2 simulations presented here are slightly different from the simulations of Stockwell et al. [1990] because the description of the chamber radical sources, other wall effects, actinic flux was updated to the chamber model developed by Carter et al. [1995]. It should be noted that the original chamber runs used by Stockwell et al. included experiment EC-306 but it had to be dropped from our comparisons because the experiment had been removed from the reevaluated data set.

Figures 3, 4, and 5 show profiles of $\mathrm{NO}_{x}$, ozone, and the aromatics toluene and xylene, respectively, for experiment EC237, which consistcd of the photolysis of a mixture of nitrogen oxides and a complex mixture of reactive organic species: 


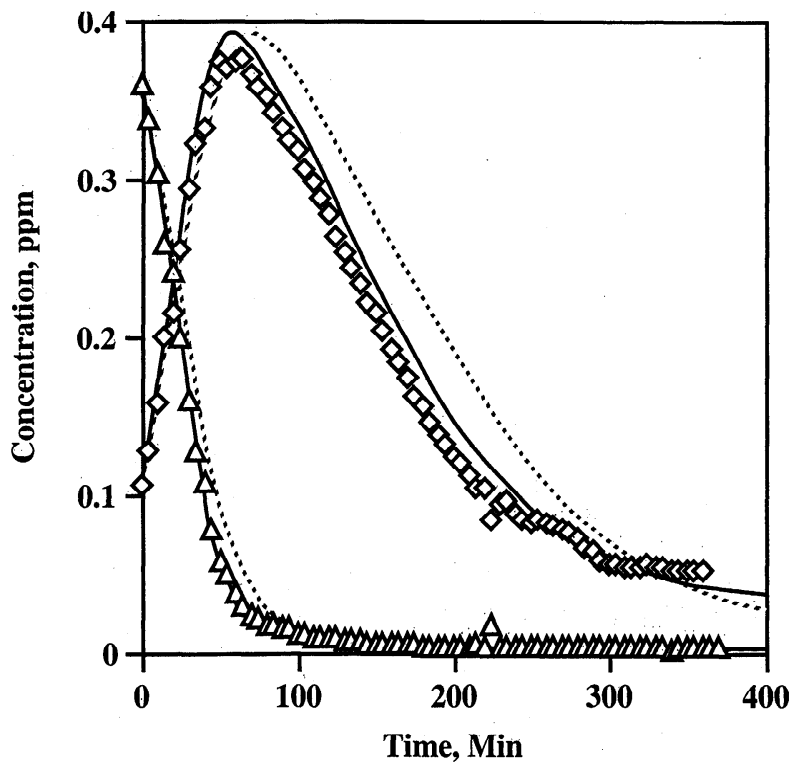

Figure 3. Predicted and observed concentration profiles of $\mathrm{NO}_{x}$ for SAPRC environmental chamber experiment EC-237. Triangles represent experimental values of NO; diamonds represent experimental $\mathrm{NO}_{2}$; solid lines represent RACM simulations; dotted line represents RADM2 simulations.

ethene, propene, t-2-butene, $n$-butene, 2,3-dimethylbutene, toluene, and $m$-xylene [Carter et al., 1995]. Experiment EC-237 is a "benchmark" case that has been presented by several mechanism developers [McRae et al., 1982; Lurmann et al., 1986; Stockwell et al., 1990]. The experiment provides a clear illustration of the chemistry of ozone formation. Figure 3 shows that $\mathrm{NO}$ is converted to $\mathrm{NO}_{2}$ which is due to the reaction of peroxy radicals with $\mathrm{NO}$. The $\mathrm{NO}_{2}$ photolyzes to produce ozone, while the reduction in NO concentrations decreases the

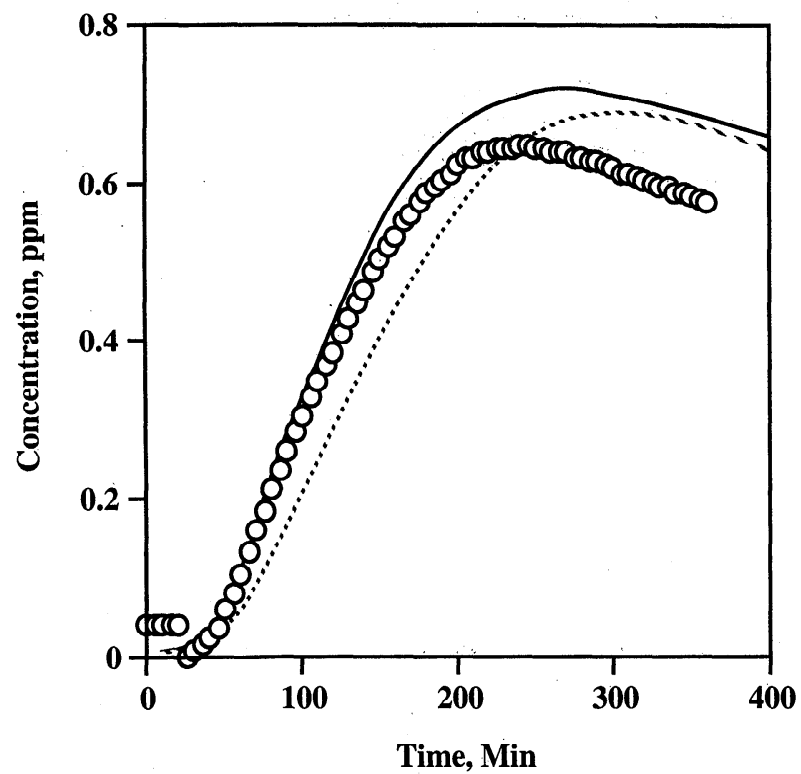

Figure 4. Predictcd and observed concentration profiles of ozone for SAPRC environmental chamber experiment EC237. Circles represent experimental values of $\mathrm{O}_{3}$; solid line represents RACM simulations; dotted line represents RADM2 simulations. rate of the $\mathrm{O}_{3}+\mathrm{NO}$ reaction. Therefore the net effect of the $\mathrm{NO}$ to $\mathrm{NO}_{2}$ conversions is to increase ozone concentrations (Figure 4).

The profiles of $\mathrm{NO}_{x}$ and ozone and the timing of the maxima of $\mathrm{NO}_{2}$ and ozone are better fit by the new mechanism than by RADM2 when the simulations are made with the revised chamber model of Carter et al. [1995]. For experiment EC-237 the NO-to- $\mathrm{NO}_{2}$ conversion rate predicted by RACM is slightly greater than the rate predicted by RADM2. The timing of the maximum $\mathrm{NO}_{2}$ concentration predicted by RACM is earlier and in better agreement with the chamber data than the RADM2 calculation.

Figure 4 shows the comparison of simulated ozone concentrations with the experimental values for EC-237. The RACM profile shows an ozone production rate more consistent with the chamber data than that predicted by RADM2. The peak concentration predicted by the RACM mechanism is slightly higher than predicted by the RADM2 mechanism. However, both predicted ozone profiles are well within $\pm 30 \%$ of the experimental ozone concentration. The timing of the ozone peak predicted by RACM is somewhat later than the experimental value but is in better agreement than the RADM2 calculations.

The predicted and measured concentrations of toluene and xylene are in good agreement for both mechanisms for these chamber conditions (Figure 5). This agreement indicates that for this experiment both mechanisms accurately predict the HO concentration because the only processes which reduce aromatic concentrations are reaction with $\mathrm{HO}$ and dilution in the chamber.

Tables 9 and 10 summarize the calculated and observed maximum $\mathrm{O}_{3}$ and $\mathrm{NO}_{2}$ concentrations and the time at which they occur for all 20 of the modeled EC experiments. In a few cases the experiments were concluded before the ozone reached a maximum or the simulations did not predict a ozone maximum during the time of the experiment. For these cases



Figure 5. Predicted and observed concentration profiles of toluene for SAPRC environmental chamber experiment EC237. Circles represent experimental values of toluene; squares represent xylene; solid lines represent RACM simulations; dotted lines represent RADM2 simulations. 
the timing of the ozone maximum in Table 9 is reported as "greater than experimental time," and the maximum ozone concentration is the concentration at the end of the experiment. These cases are included in Figure 6, showing the peak $\mathrm{O}_{3}$ concentrations observed and predicted by the two mechanisms, but they are not included in Figure 7 in which the timing of the peak $\mathrm{O}_{3}$ concentrations is compared. Overall, the RACM mechanism generally predicts higher peak ozone concentrations than those predicted by RADM2. For the peak ozonc concentrations the mean normalized deviations of the RACM and RADM2 calculated values from the EC experimental values are 19 and $13 \%$, respectively.

The timing of the ozone maximum is better represented by the RACM mechanism for most cases, except for a few cases with an aromatic hydrocarbon as the principal component (EC-331, EC-344, and EC-345). If these cases are included, the mean of the normalized deviations of the RACM calculated timing of the peak ozone concentrations from the EC experimental values is $30 \%$, while RADM2 gives a value of $27 \%$. For EC-340 the agreement between the final experimental and simulated ozone concentrations was better for the RACM mechanism. If runs EC-331, EC-340, EC-344, and EC-345 are excluded, the mean normalized deviations are 11 and $25 \%$ for RACM and RADM2, respectively.

For cxperiment EC-331 the RACM mechanism predicted the toluene profile better than the RADM2. Because toluene concentrations in the chamber were affected only by $\mathrm{HO}$ reaction and dilution, this suggests that the RACM mechanism predicted $\mathrm{HO}$ concentrations better than the RADM2 mechanism. For EC-344 run where $m$-xylene was the major aromatic compound, the ozone profile and xylene profiles were both simulated better by the original RADM2.

However, simulations of aromatic photo-oxidation experiments made in another SAPRC chamber (the evacuatable Teflon chamber (ETC)) gave better agreement between model

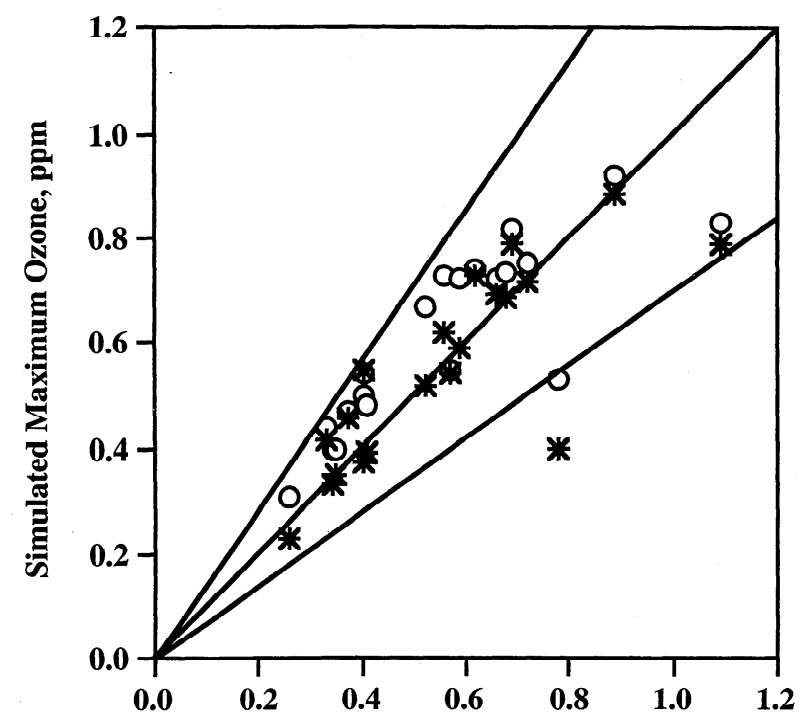

Experimental Maximum Ozone, ppm

Figure 6. Maximum ozone concentrations predicted by RACM and RADM2 mechanisms plottcd against SAPRC experimental values. Circles refer to RACM mechanism; asterisks refer to RADM2. Middle line is the 1-1 line; top and bottom lines represent $\pm 30 \%$ deviation from the 1-1 line.

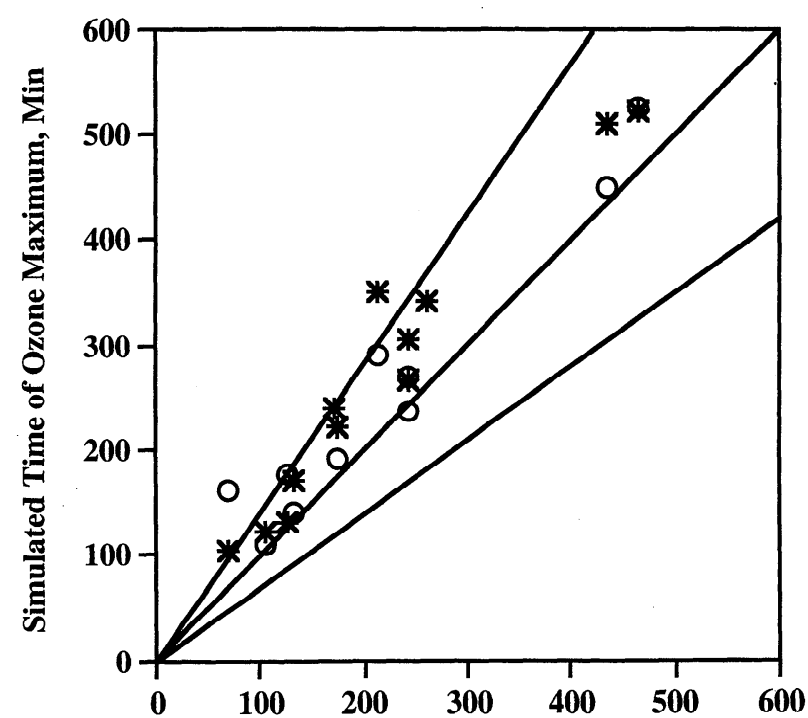

Experimental Time of Ozone Maximum, Min

Figure 7. Time of the maximum of the ozone concentrations predicted by RACM and RADM2 mechanisms plotted against SAPRC experimental values. Only experiments where an ozone peak was observed are included. Circles refer to RACM mechanism; asterisks refer to RADM2. Middle line is the 1-1 line; top and bottom lines represent $\pm 30 \%$ deviation from the 1-1 line.

and experiment for the RACM mechanism, but these experiments were performed under black light illumination and low relative humidity $(\sim 5 \%)$. Furthermore, the chamber studies showed that the RADM cresol mechanism produced too much ozone and that the timing of the peak ozone was late. The revised RACM mechanism predicts the timing of the ozone peak better, but the amount of ozone production from cresol requires improvement. Although the aromatic chemistry in the RACM mechanism is based on more recent data than the RADM2 mechanism, future versions will require additional research.

Both mechanisms predict peak $\mathrm{NO}_{2}$ concentrations very well (Figure 8). The mean normalized deviations of $\mathrm{NO}_{2}$ peak concentrations from the EC chamber experimental measurements for RACM and RADM2 are 10 and 9\%, respectively. The timing of the $\mathrm{NO}_{2}$ peak concentration predicted by RACM and RADM2 are very similar (Figure 9). The timing of the simulated $\mathrm{NO}_{2}$ maxima is later in most cases, but the overall performance of RACM is only slightly improved (Figure 9). The mean normalized deviations of timing of the $\mathrm{NO}_{2}$ peak concentrations for RACM and RADM2 are both near $3 \%$. The ability of the mechanism to correctly simulate the NO and $\mathrm{NO}_{2}$ profiles suggests that not only the $\mathrm{HO}$ concentrations but also the underlying acyl peroxy radical concentrations and their effect on the chemistry $\mathrm{NO}_{2}$ are well simulated because under thesc conditions, significant amounts of PAN and PAN analogs are produced.

\section{Comparison of the RACM and RADM2 Mechanisms}

The concentrations of $\mathrm{O}_{3}, \mathrm{H}_{2} \mathrm{O}_{2}, \mathrm{H}_{2} \mathrm{SO}_{4}, \mathrm{HNO}_{3}$, and PAN predicted by the RACM and the RADM2 mechanisms were compared. The purpose of this comparison was to allow an 


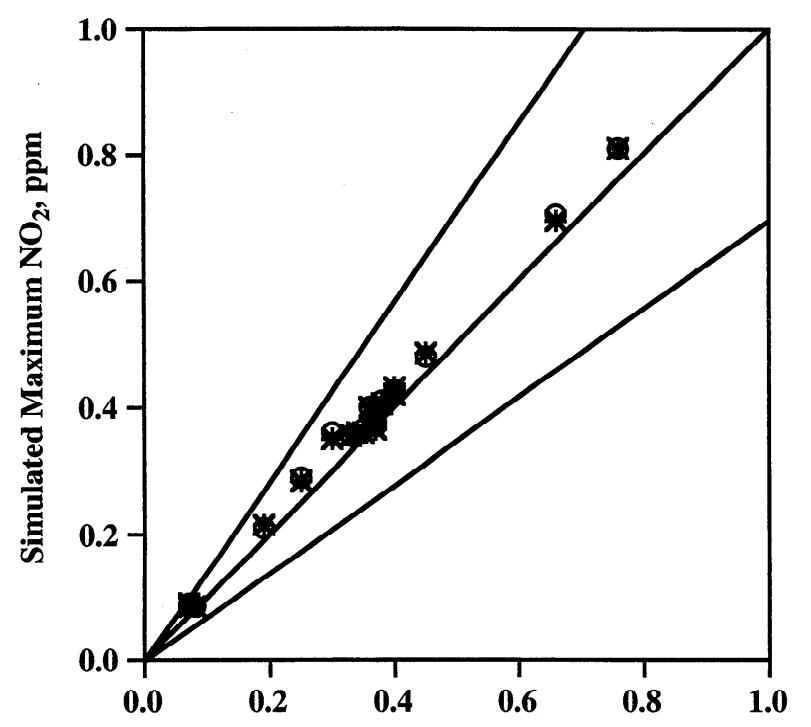

Experimental Maximum $\mathrm{NO}_{2}, \mathrm{ppm}$

Figure 8. Maximum $\mathrm{NO}_{2}$ concentrations predicted by RACM and RADM2 mechanisms plotted against SAPRC experimental values. Circles refer to RACM mechanism; asterisks refer to RADM2. Middle line is the 1-1 line; top and bottom lines represent $\pm 30 \%$ deviation from the $1-1$ line.

assessment of the effect of the revisions on the calculated concentrations of species that are of particular interest to the developers of regional air quality models. No emissions, dilution, or deposition were included in the first 18 cases. The impacts of emissions on the relative performance of the mechanisms were assessed through cases 19-21 discussed below.

The first 18 simulations are from an extensive mechanism intercomparison study [Stockwell and Lurmann, 1989]. The

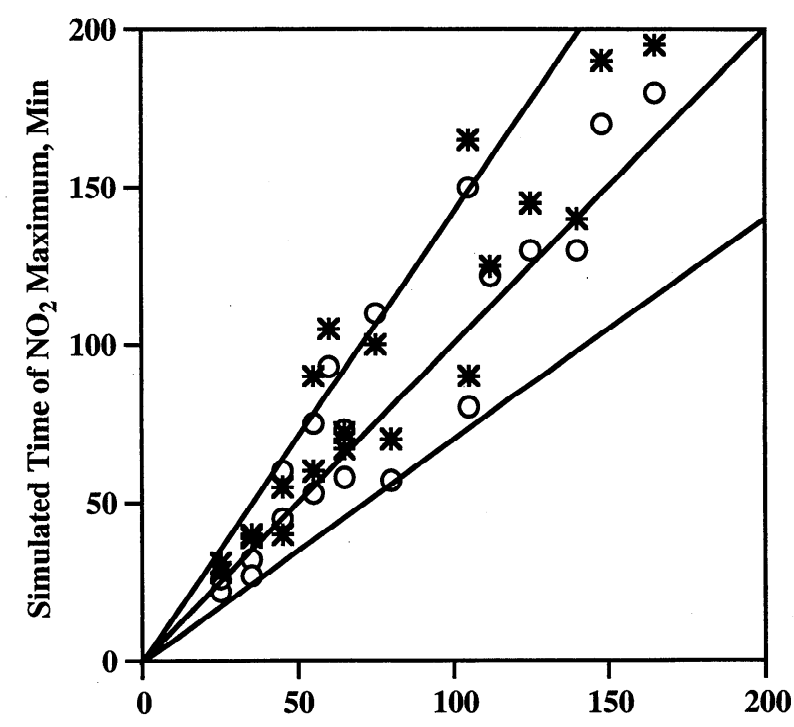

Experimental Time of $\mathrm{NO}_{2}$ Maximum, Min

Figure 9. Time of the maximum of the $\mathrm{NO}_{2}$ concentrations predicted by RACM and RADM 2 mechanisms plotted against SAPRC experimental values. Circles refer to RACM mechanism; asterisks refer to RADM2. Middle line is the 1-1 line; top and bottom lines represent $\pm 30 \%$ deviation from the $1-1$ line. simulations were performed for typical rural and urban conditions in a manner similar to a comparison of the first and second version of the RADM mechanisms [Stockwell et al., 1990]. Two days were simulated for summer surface conditions starting and stopping at $0600 \mathrm{LT}$. The simulations employed diurnally varying solar radiation for $40^{\circ}$ latitude. The fluxes are calculated from a radiative transfer model based on the deltaEddington technique [Joseph et al., 1976; Madronich, 1987] as described by Stockwell et al. [1990]. The original and updated photolysis rates were used for the RADM2 and the RACM mechanisms, respectively.

The composition of the rural and urban mixtures shown in Table 11 were used, and Table 12 shows the initial concentrations for these simulations. The rural simulations have initial $\mathrm{NO}_{x}$ concentrations ranging from 0.5 to $5 \mathrm{ppb}$, total nonmethane organic compound (NMOC) concentrations ranging from 10 to $100 \mathrm{ppb}$ C. The RADM2 aggregation factors were used for both mechanisms. The rural initial $\mathrm{NMOC} / \mathrm{NO}_{x}$ ratios ranged from 2 to 200. The urban simulations have initial $\mathrm{NO}_{x}$ concentrations ranging from 10 to $100 \mathrm{ppb}$, initial VOC concentrations ranging from 100 to $1000 \mathrm{ppb} \mathrm{C}$, and initial $\mathrm{NMOC} / \mathrm{NO}_{x}$ ratios ranging from 1 to 100 .

The RACM and RADM2 mechanisms predict similar $\mathrm{O}_{3}$ concentrations for typical atmospheric conditions (Table 13). The average ratio of $\mathrm{O}_{3}$ predicted by RACM to that predicted by RADM2 is 1.08 . The RACM mechanism predicts higher peak $\mathrm{O}_{3}$ concentrations for 11 of the 18 cases. The $\mathrm{O}_{3}$ predictions for the rural cases are all in excellent agreement. The RACM mechanism predicts more $\mathrm{O}_{3}$ than the RADM2 mechanism for all but one of the urban cases with the greatest diffcrences occurring for cases 11,12 , and 15 . The urban cases have a greater proportion of highly reactive alkenes and aromatics than the rural cases, and therefore the urban cases are

Table 11. Nonmethane Organic Composition Used for Cases 1-18

\begin{tabular}{|c|c|c|c|c|}
\hline \multirow[b]{2}{*}{$\begin{array}{l}\text { Organic Group/ } \\
\text { Compound }\end{array}$} & \multicolumn{2}{|c|}{ Rural Summer Surface } & \multicolumn{2}{|c|}{ Urban Summer Surface } \\
\hline & $\begin{array}{c}\text { Fraction } \\
\text { of } \\
\text { Group }_{\%}^{a}\end{array}$ & $\begin{array}{c}\text { Fraction of } \\
\text { Nonmethane } \\
\text { Organic } \\
\text { Carbon, }{ }^{a} \%\end{array}$ & $\begin{array}{l}\text { Fraction } \\
\text { of } \\
\text { Group, } \\
\%\end{array}$ & $\begin{array}{c}\text { Fraction of } \\
\text { Nonmethane } \\
\text { Organic } \\
\text { Carbon, a \% }\end{array}$ \\
\hline Alkanes & & 63.0 & & 55.0 \\
\hline Ethane & 25 & 15.8 & 10 & 5.5 \\
\hline Propane & 20 & 12.6 & 15 & 8.3 \\
\hline$n$-butane & 15 & 9.5 & 15 & 8.3 \\
\hline$n$-pentane & 15 & 9.5 & 15 & 8.3 \\
\hline$n$-hexane & 10 & 6.3 & 15 & 8.3 \\
\hline$n$-heptane & 10 & 6.3 & 15 & 8.3 \\
\hline$n$-octanc & 5 & 3.2 & 15 & 8.3 \\
\hline Alkenes & & 15.0 & & 20.0 \\
\hline Ethene & 40 & 6.0 & 35 & 7.0 \\
\hline Propene & 20 & 3.0 & 40 & 8.0 \\
\hline tert-2-butene & 0 & 0 & 25 & 5.0 \\
\hline Isoprene & 40 & 6.0 & 0 & 0 \\
\hline Aromatics & & 20.0 & & 20.0 \\
\hline Benzene & 50 & 10.0 & 20 & 4.0 \\
\hline Toluene & 35 & 7.0 & 40 & 8.0 \\
\hline$m$-xylene & 15 & 3.0 & 25 & 5.0 \\
\hline Mesitylene & 0 & 0 & 15 & 3.0 \\
\hline Aldehydes & & 2.0 & & 5.0 \\
\hline Formaldehyde & 50 & 1.0 & 50 & 2.5 \\
\hline Acetaldehyde & 50 & 1.0 & 50 & 2.5 \\
\hline
\end{tabular}

From Stockwell et al. [1990].

${ }^{a}$ All organic fractions are calculated on a carbon basis. 
more strongly affected by the revisions to the aromatic chemistry and the nighttime $\mathrm{NO}_{3}+$ alkene chemistry.

The RACM mechanism predicts less $\mathrm{H}_{2} \mathrm{O}_{2}$ than the RADM2 mechanism for 15 of the cases due to the revisions to the peroxy radical chemistry [Kirchner and Stockwell, 1996a]. The predicted $\mathrm{H}_{2} \mathrm{O}_{2}$ concentrations agree well for the cases which produce large amounts $\mathrm{H}_{2} \mathrm{O}_{2}$, but the mechanisms show their greatest differences for the cases 16 and 17 where both predicted little $\mathrm{H}_{2} \mathrm{O}_{2}$. The average ratio of $\mathrm{H}_{2} \mathrm{O}_{2}$ predicted by RACM to that predicted by RADM2 is 0.90 if these two cases are excluded and 0.99 if they are included.

The RACM mechanism predicts higher $\mathrm{H}_{2} \mathrm{SO}_{4}$ concentrations than the RADM2 for all 18 cases; the average predicted peak $\mathrm{H}_{2} \mathrm{SO}_{4}$ concentration ratios of RACM to RADM2 are 1.20. This also shows that the RACM mechanism predicts higher $\mathrm{HO}$ concentrations for these rural and urban conditions because the reaction of $\mathrm{HO}$ with $\mathrm{SO}_{2}$ is only gas-phase reaction which produces $\mathrm{H}_{2} \mathrm{SO}_{4}$ in the mechanisms. The differences between the RACM and RADM 2 mechanisms are greatest for the urban cases, and these differences are greater for those cases with the higher initial $\mathrm{NMOC} / \mathrm{NO}_{x}$ ratios.

The RACM mechanism predicts slightly higher $\mathrm{HNO}_{3}$ concentrations than the RADM2 for 14 of the 18 cases. The

Table 12. Initial Conditions for Mechanism Intercomparison Cases 1-18

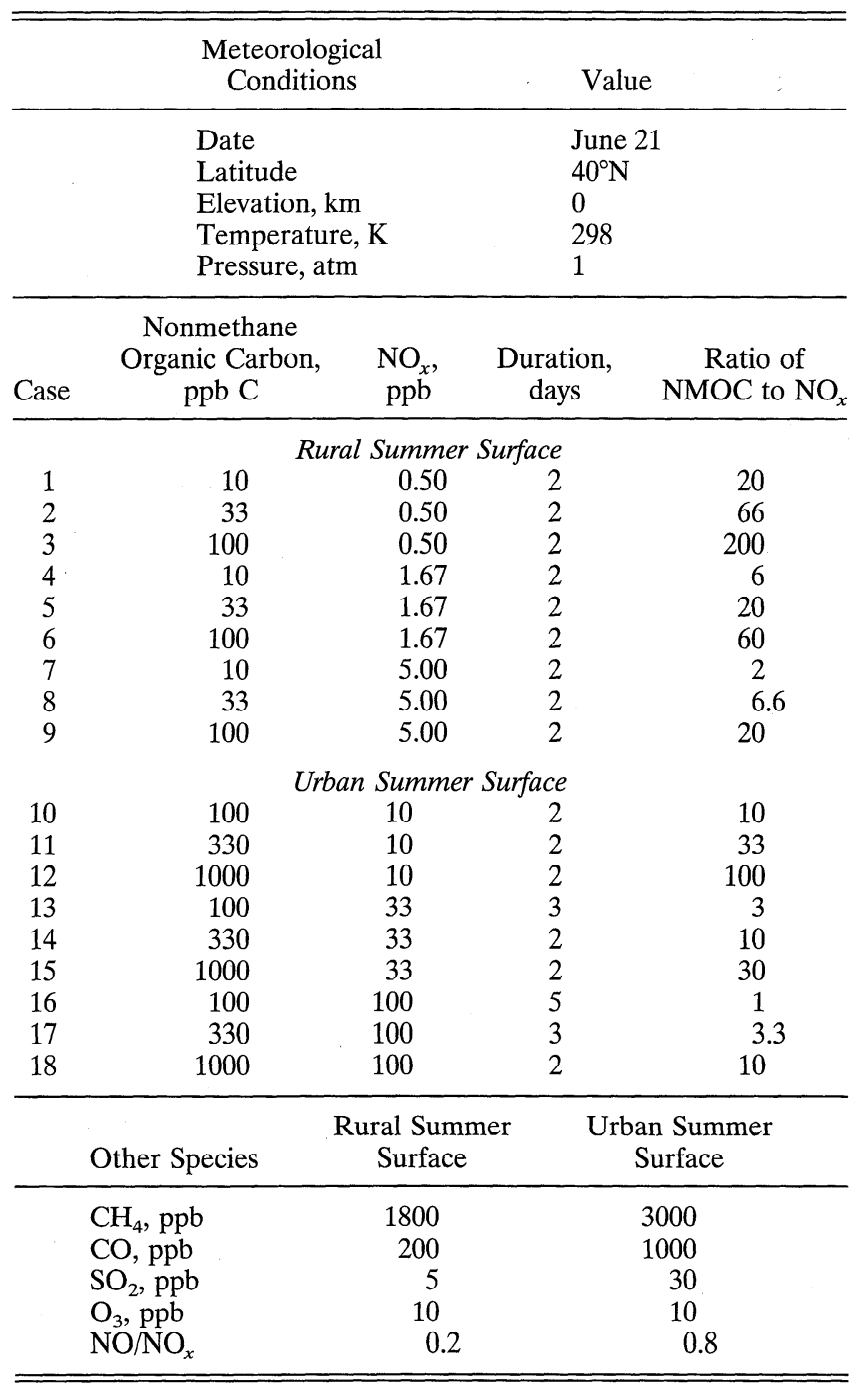

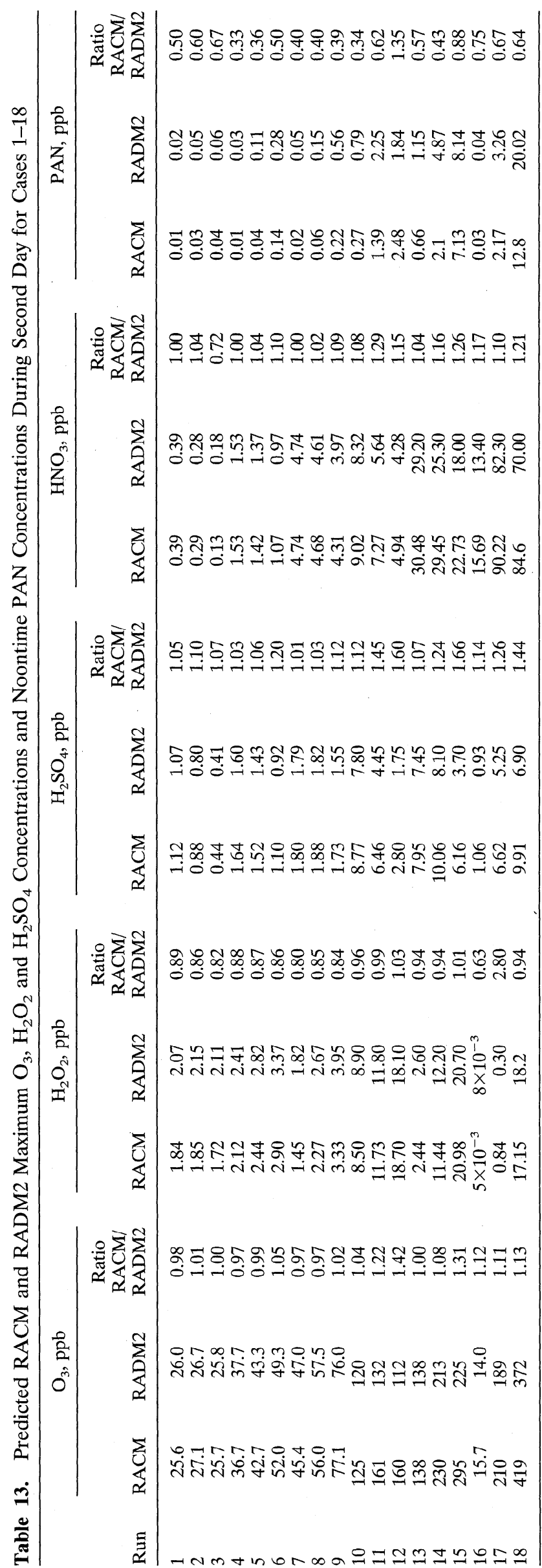


Table 14. Initial Conditions for Cases 19-21

\begin{tabular}{|c|c|c|c|c|c|}
\hline \multirow[b]{2}{*}{ Compound } & \multicolumn{2}{|c|}{ Case 19} & \multicolumn{2}{|c|}{ Case 20} & \multirow[b]{2}{*}{$\begin{array}{c}\text { Case } 21 \text { Initial } \\
\text { Concentrations } \\
\text { ppb }\end{array}$} \\
\hline & $\begin{array}{c}\text { Initial } \\
\text { Concentrations, } \\
\mathrm{ppb}\end{array}$ & $\begin{array}{c}\text { Emissions, } \\
\mathrm{ppt} / \mathrm{min}\end{array}$ & $\begin{array}{c}\text { Initial } \\
\text { Concentrations, } \\
\text { ppb }\end{array}$ & $\begin{array}{c}\text { Emissions, } \\
\mathrm{ppt} / \mathrm{min}\end{array}$ & \\
\hline \multicolumn{6}{|c|}{ Inorganics } \\
\hline NO & 0.2 & 2.6 & 0.02 & 0.26 & $\cdots$ \\
\hline $\mathrm{NO}_{2}$ & 0.5 & $\ldots$ & 0.05 & $\ldots$ & 1.0 \\
\hline $\mathrm{HNO}_{3}$ & 0.1 & $\cdots$ & 0.01 & $\cdots$ & 0 \\
\hline $\mathrm{H}_{2} \mathrm{O}$ & $1 \times 10^{7}$ & $\cdots$ & $1 \times 10^{7}$ & $\cdots$ & $1.6 \times 10^{7}$ \\
\hline $\mathrm{O} 3$ & 50 & $\ldots$ & 30 & $\cdots$ & 30 \\
\hline $\mathrm{H}_{2} \mathrm{O}_{2}$ & 2.0 & $\cdots$ & 0.2 & $\cdots$ & $\cdots$ \\
\hline $\mathrm{SO}_{2}$ & $\cdots$ & 0.52 & $\cdots$ & 0.052 & 1.0 \\
\hline $\mathrm{CO}$ & 200 & 5.7 & 104 & 0.57 & 104 \\
\hline \multicolumn{6}{|c|}{ Alkanes } \\
\hline $\mathrm{CH}_{4}$ & 1700 & $\cdots$ & 1700 & $\cdots$ & 1700 \\
\hline ETH & $\cdots$ & 0.24 & $\cdots$ & 0.024 & 1.2 \\
\hline $\mathrm{HC} 3$ & $\cdots$ & 2.6 & $\cdots$ & 0.26 & 1.3 \\
\hline HC5 & $\cdots$ & 0.76 & $\cdots$ & 0.076 & 2.1 \\
\hline $\mathrm{HC} 8$ & $\cdots$ & 0.45 & $\cdots$ & 0.045 & 1.1 \\
\hline \multicolumn{6}{|c|}{ Alkenes } \\
\hline EITE & $\cdots$ & 0.46 & $\cdots$ & 0.046 & 0.66 \\
\hline OLI & $\cdots$ & 0.19 & $\cdots$ & 0.019 & 0.42 \\
\hline OLT & $\cdots$ & 0.22 & $\ldots$ & 0.022 & 0.27 \\
\hline ISO & $\cdots$ & $\cdots$ & $\cdots$ & $\cdots$ & 0.5 \\
\hline \multicolumn{6}{|c|}{ Aromatics } \\
\hline TOL & $\cdots$ & 0.57 & $\cdots$ & 0.057 & 1.5 \\
\hline XYL & $\cdots$ & 0.52 & $\cdots$ & 0.052 & 0.07 \\
\hline \multicolumn{6}{|c|}{ Carbonyls } \\
\hline HCHO & .1 .0 & 0.14 & 0.1 & 0.014 & 2.1 \\
\hline ALD & $\cdots$ & 0.036 & $\cdots$ & 0.0036 & 1.0 \\
\hline KET & $\ldots$ & 0.32 & $\cdots$ & 0.032 & 1.0 \\
\hline PAN & $\cdots$ & & $\cdots$ & $\cdots$ & 1.0 \\
\hline
\end{tabular}

From Kirchner and Stockwell [1996a].

average predicted peak $\mathrm{HNO}_{3}$ concentrations ratios is 1.08 . The greatest differences are seen for the urban cases and the differences arc greatest for those cases with the higher initial $\mathrm{NMOC} / \mathrm{NO}_{x}$ ratios. The concentrations are affected by the differences between the predicted $\mathrm{HO}$ concentrations and the differences in the nighttime $\mathrm{NO}_{3}$ chemistry which affects the predicted nitrogen balance.

The differences in predicted PAN concentrations are very significant, and these results are in accord with our previous results [Kirchner and Stockwell, 1996a]. The RACM mechanism predicts less PAN at noon on the second day for all cases except case 12 . The average predicted noontime PAN concentration ratios of RACM to RADM2 are 0.58. The increased rates of reaction of the $\mathrm{ACO}_{3}$ radical with $\mathrm{NO}$ and the additional reaction of $\mathrm{ACO}_{3}$ with $\mathrm{NO}_{3}$ cause the predicted concentrations of PAN predicted by the RACM mechanism to be significantly reduced. This illustrates the importance of the revisions to the $\mathrm{NO}_{3}$ and peroxy radical chemistry.

To further determine the cause of the observed differences between RACM and RADM2, three additional cases were simulated (Table 14). The first two cases are based upon conditions used in an international intercomparison of chemical mechanisms [Poppe et al., 1996], and all of these cases were used to determine the effect of revisions to peroxy radical chemistry on predicted concentrations [Kirchner and Stockwell, 1996a]. Cases 19, 20, and 21 were cases 1, 2, and 3, respectively, of Kirchner and Stockwell [1996a]. The same meteorological conditions were used for these simulations as were used for cases 1-18. Emissions are included for cases 19 and 20. Case 19 represents a somewhat polluted atmosphere with emissions of $\mathrm{NO}_{x}$ and organic compounds. Case 20 represents a relatively cleaner atmosphere with the initial $\mathrm{NO}_{x}$ and organic compound concentrations and emission rates reduced by a factor of 10 from those of case 19 . Case 21 represents the situation of aged air mixing with rural air. The three cases were simulated using the RADM2 mechanism, the RADM2 mechanism with updated peroxy radical chemistry (run D of Kirchner and Stockwell [1996a]), and the new RACM mechanism.

Table 15 shows the results of this intercomparison for cases 19 to 21 . The effect of the revisions to peroxy radical chemistry was discussed by Kirchner and Stockwell [1996a]; therefore we focus the differences between the RACM and RADM2 mechanisms with revised peroxy radical chemistry (RADM2-D). The $\mathrm{O}_{3}$ concentrations were not strongly affected by the revisions. However, the RACM mechanism predicted nighttime $\mathrm{HO}$ and $\mathrm{HO}_{2}$ concentrations that were much greater than those of RADM2-D. The ratios of the nighttime HO concentrations predicted by the RACM mechanism to that predicted by the RADM2-D mechanism were $3.34,2.90$, and 2.04 , for cases 19,20 , and 21 , respectively. The ratios of the nighttime $\mathrm{HO}_{2}$ concentrations predicted by the RACM mechanism to that predicted by the RADM2-D mechanism were $1.39,1.49$, and 1.28 for cases 19,20 , and 21 , respectively. The most important reason for the greater nighttime $\mathrm{HO}$ concentrations predicted by the RACM mechanism is the revised product composition of the reaction of $\mathrm{HO}_{2}$ with $\mathrm{NO}_{3}$. For case 19 the 
Table 15. Simulation Results for Cases 19-21

\begin{tabular}{|c|c|c|c|c|c|c|c|c|c|c|c|c|c|c|}
\hline \multirow[b]{2}{*}{ Run } & \multirow[b]{2}{*}{ Time $^{\mathrm{a}}$} & \multirow[b]{2}{*}{ Units } & \multicolumn{4}{|c|}{ Case $19^{\mathrm{a}}$} & \multicolumn{4}{|c|}{ Case $20^{\mathrm{a}}$} & \multicolumn{4}{|c|}{ Case $21^{\mathrm{a}}$} \\
\hline & & & $\mathrm{RADM} 2^{\mathrm{b}}$ & RADM2-D ${ }^{c}$ & RACM & $\begin{array}{c}\text { RACM/ } \\
\text { RADM2-D }\end{array}$ & RADM $^{\mathrm{b}}$ & RADM2-D ${ }^{c}$ & RACM & $\begin{array}{c}\text { RACM/ } \\
\text { RADM2-D }\end{array}$ & $\mathrm{RADM}^{\mathrm{b}}$ & RADM2- $D^{c}$ & RACM & $\begin{array}{c}\text { RACM/ } \\
\text { RADM2-D }\end{array}$ \\
\hline $\mathrm{O}_{3}$ & overall & $\mathrm{ppb}$ & 150 & 146 & 148 & 1.01 & 51.4 & 51.1 & 50.1 & 0.98 & 58.4 & 57.9 & 57.2 & 0.99 \\
\hline PAN & day & $\mathrm{ppb}$ & 2.3 & 1.1 & 1.10 & 1.00 & 0.11 & 0.05 & 0.04 & 0.80 & 0.14 & 0.06 & 0.04 & 0.67 \\
\hline PAN & night & $\mathrm{ppb}$ & 2.2 & 0.7 & 0.64 & 0.91 & 0.071 & 0.016 & 0.012 & 0.75 & 0.12 & 0.02 & 0.01 & 0.50 \\
\hline $\mathrm{H}_{2} \mathrm{O}_{2}$ & overall & $\mathrm{ppb}$ & 5.7 & 4.5 & 4.03 & 0.90 & 2.67 & 2.50 & 2.21 & 0.88 & 3.48 & 3.21 & 2.96 & 0.92 \\
\hline Methyl hydroperxide & overall & $\mathrm{ppb}$ & 0.98 & 0.73 & 0.88 & 1.21 & 0.55 & 0.48 & 0.64 & 1.33 & 0.96 & 0.90 & 1.15 & 1.28 \\
\hline Higher hydroperoxides & overall & $\mathrm{ppb}$ & 1.2 & 2.02 & 2.13 & 1.05 & 0.15 & 0.30 & 0.36 & 1.20 & 0.33 & 0.60 & 0.75 & 1.25 \\
\hline Peroxoacetic acid & overall & $\mathrm{ppb}$ & 0.72 & 0.26 & 0.29 & 1.12 & 0.13 & 0.05 & 0.05 & 1.00 & 0.34 & 0.13 & 0.13 & 1.00 \\
\hline Higher aldehydes & day & $\mathrm{ppb}$ & 1.37 & 1.31 & 0.91 & 0.69 & 0.171 & 0.159 & 0.107 & 0.67 & 0.25 & 0.32 & 0.15 & 0.47 \\
\hline Higher aldehydes & night & ppb & 1.00 & 1.18 & 0.814 & 0.69 & 0.160 & 0.163 & 0.101 & 0.62 & 0.26 & 0.31 & 0.15 & 0.48 \\
\hline NO & day & ppt & 132 & 155 & 182 & 1.17 & 53.4 & 54 & 57.7 & 1.07 & 21 & 16 & 18.4 & 1.15 \\
\hline $\mathrm{NO}_{3}$ & night & ppt & 301 & 182 & 183 & 1.01 & 69 & 45 & 38 & 0.84 & 69 & 27 & 29 & 1.07 \\
\hline $\mathrm{HO}$ & day & ppt & 0.308 & 0.303 & 0.365 & 1.20 & 0.290 & 0.296 & 0.327 & 1.10 & 0.30 & 0.30 & 0.34 & 1.13 \\
\hline $\mathrm{HO}$ & night & ppt & 0.0009 & 0.0029 & 0.0099 & 3.41 & 0.00026 & 0.00069 & 0.00185 & 2.68 & 0.00045 & 0.00091 & 0.00170 & 1.87 \\
\hline $\mathrm{HO}_{2}$ & day & ppt & 37 & 33 & 34 & 1.03 & 24.5 & 23.8 & 23.6 & 0.99 & 25.5 & 25.2 & 25.7 & 1.02 \\
\hline $\mathrm{HO}_{2}$ & night & ppt & 0.6 & 2.3 & 2.8 & $1: 22$ & 0.16 & 0.45 & 0.59 & 1.31 & 0.30 & 0.60 & 0.62 & 1.03 \\
\hline Methyl peroxy radicals & day & $\mathrm{ppt}$ & 15.7 & 14.3 & 12.4 & $0: 87$ & 13.1 & 12.8 & 11.4 & 0.89 & 23 & 23 & 20 & 0.87 \\
\hline Methyl peroxy radicals & night & $\mathrm{ppt}$ & 16.5 & 4.2 & 3.95 & 0.94 & 2.6 & 0.8 & 0.94 & 1.18 & 6.8 & 1.7 & 1.5 & 0.88 \\
\hline Actetyl peroxy radicals & day & ppt & 10.9 & 3.5 & 2.98 & 0.85 & 2.7 & 0.9 & 0.688 & 0.76 & 6.7 & 2.2 & 1.5 & 0.68 \\
\hline Actetyl peroxy radicals & night & $\mathrm{ppt}$ & 9.1 & 1.1 & 1.0 & 0.91 & 1.18 & 0.16 & 0.13 & 0.81 & 3.7 & 0.4 & 0.2 & 0.50 \\
\hline OLN or OLNN+OLND & night & $\mathrm{ppt}$ & 40 & 1.0 & 0.96 & 0.96 & 6.8 & 0.30 & 0.40 & 1.33 & 0.019 & 0.0006 & 0.0008 & 1.33 \\
\hline
\end{tabular}

${ }^{\mathrm{a}}$ Overall represents for cases 19 and 20 the highest value between sunrise of the fifth day and sunrise of the sixth day and for case 21 , the highest value between sunset of the second day and sunsct


for cases 19 and 20, fifth night, $0130 \mathrm{LT}$; and for case 21, second night, $0130 \mathrm{LT}$.

Original RADM2 with $\mathrm{N}_{2} \mathrm{O}_{5}$ hydrolysis rate constant set to 0

'Only peroxy radical reactions updated (run D of Kirchner and Stockwell [1996a]). 
$\mathrm{HO}_{2}+\mathrm{NO}_{3}$ reaction becomes the most important $\mathrm{HO}$ source during the night, and for cases 20 and 21 this reaction occurs at a rate that is about 75 and $50 \%$ the rate of the $\mathrm{O}_{3}+\mathrm{HO}_{2}$ reaction.

The higher nighttime $\mathrm{HO}$ concentrations increase the importance of their reactions during the nighttime. For case 19 a counter species analysis [Leone and Seinfeld, 1985] of the RACM simulation showed that the fraction of organic compounds which react with $\mathrm{HO}$ slightly exceed the fraction reacting with $\mathrm{NO}_{3}$. The fraction of organic compounds which react with $\mathrm{O}_{3}$ was significantly less than the fraction reacting with either $\mathrm{HO}$ or $\mathrm{NO}_{3}$ during the nighttime hours. On the fifth night of this simulation, for example, the amount of HCHO that reacted with $\mathrm{NO}_{3}$ is the same as the amount that reacted with $\mathrm{HO}$. There are additional slow reactions of $\mathrm{NO}_{3}$ with alkanes which are not included in the RACM mechanism which may increase the relative fraction of organic compounds that react with $\mathrm{NO}_{3}$ and slightly decrease the relative importance of the $\mathrm{HO}$ reaction. However, the comparison of the RACM mechanism with the RADM2-D mechanism shows that during the nighttime the reaction of organic species with $\mathrm{HO}$ can be important. The revised nighttime production of $\mathrm{HO}$ now included in the RACM mechanism produce greater $\mathrm{HO}_{2}$ and organic peroxy radical concentrations.

The revisions incorporated into the aromatic oxidation scheme in the RACM mechanism lead to greater $\mathrm{NO}_{x}$ concentrations. Comparison of the RACM simulations with the RADM2-D simulations shows that this increase is due to lower organic nitrate concentrations predicted by the RACM mechanism. Although the organic nitratc photolysis frequency is higher in the RACM mechanism, the lower organic nitrate concentrations are due to lower production from aromatic oxidation. In the RADM2 and RADM2-D mechanisms, large amounts of cresols are produced from the oxidation of aromatic species. During the night the cresol rapidly reacts with $\mathrm{NO}_{3}$ to produce intermediates that react with $\mathrm{NO}_{2}$ to form organic nitrate. Organic nitrates are produced in the RACM mechanism through the reaction of $\mathrm{PHO}$ with $\mathrm{NO}_{2}$. Phenoxy radicals are produced from the reaction of cresol with $\mathrm{NO}_{3}$ and partially from the reaction of cresol with HO. In the RACM mechanism the cresol yields are much lower than in the RADM2 and RADM2-D mechanisms; this leads to much lower concentrations of phenoxy radicals and organic nitrate.

During daytime the lower cresol yield from aromatics in combination with a faster rate of $\mathrm{HO}_{2}$ production and a reduced organic peroxy radical production rate from the reaction of $\mathrm{HO}$ with $\mathrm{HC} 3$ lead to higher daytime $\mathrm{HO}, \mathrm{HO}_{2}$, and $\mathrm{NO}_{x}$ concentrations for the RACM mechanism. The revised rate parameter for the $\mathrm{HO}+\mathrm{HC} 3$ reaction is significantly lower in RACM. The increase in the HO concentrations leads to increased aldehyde (ALD) production from the hydrocarbons, and consequently, the rate of the ALD + HO reaction is increased. This produces more acetyl peroxy radicals $\left(\mathrm{ACO}_{3}\right)$, peroxy acetic acid (PAA), and PAN for the RACM mechanism relative to the RADM2-D mechanism. The concentrations of PAN and PAA are further increased because the revised rate constants for the reaction of these compounds with $\mathrm{HO}$ are lower than those used in RADM2. The increase of methyl hydrogen peroxide (OP1) concentrations results from the updated photolysis rate constant which is about $25 \%$ lower than the one used in RADM2. This leads to a significant increase in the predicted methyl hydrogen peroxide concentrations. The increase in the higher organic peroxide concentrations is much smaller because it has a much greater rate constant with $\mathrm{HO}$ which makes its loss by photolysis relatively less important.

\section{Conclusions}

The Regional Atmospheric Chemistry Mechanism is a substantially revised version of the RADM2 mechanism [Stockwell et al., 1990], with rate constants and product yields from the most recent laboratory measurements. The mechanism has been compared with the previous RADM 2 mechanism, and it has been tested against environmental chamber data. The mechanism includes 17 stable inorganic species, 4 inorganic intermediates, 32 stable organic species, and 24 organic intermediates in 237 reactions. Several inorganic reactions were added to the RACM mechanism including reactions of $\mathrm{O}\left({ }^{3} P\right)$ with nitrogen oxides and ozone, the reaction of $\mathrm{HO}$ with $\mathrm{H}_{2}$, $\mathrm{HO}$ with nitrous acid, and a few additional $\mathrm{NO}_{3}$ reactions.

It should be stressed that the RACM mechanism considers only the gas-phase chemistry of the troposphere. Therefore the reaction of $\mathrm{N}_{2} \mathrm{O}_{5}$ with $\mathrm{H}_{2} \mathrm{O}$ was dropped from the RACM gas-phase mechanism because it now appears that the $\mathrm{N}_{2} \mathrm{O}_{5}+$ $\mathrm{H}_{2} \mathrm{O}$ reaction is a heterogeneous reaction. Heterogeneous and aqueous phase reactions have a strong effect on the gas-phase chemistry, and these should be included in photochemical atmospheric chemistry models [Seinfeld, 1986]. However, much more data from both laboratory and field measurements will be required before this aspect of atmospheric chemistry is well understood.

To make the mechanism suitable for three-dimensional (3-D) models, the RACM mechanism uses grouped organic species. Aggregation factors for reactive organic species were developed according to the methods of Middleton et al. [1990] and Stockwell et al. [1990]. The organic chemistry was significantly revised. Revisions to the branching ratios for alkane decay led to significantly reduced aldehyde to ketone ratios. The decomposition and nitrate formation channels for the reactions of $\mathrm{NO}_{3}$ with alkenes are now more explicitly treated, and the relatively large amounts of nitrates resulting from the reactions of unbranched alkenes are included in the RACM mechanism. The production of $\mathrm{HO}$ from the ozonolysis of alkenes has a much greater yield in the RACM mechanism than in the RADM2 mechanism.

The aromatic chemistry has been revised to be more consistent with recent laboratory data. Many more aromatic reaction products are accounted for in the RACM mechanism. Large amounts of products from the aromatic-HO addition reactions had been ignored in the RADM2 mechanism; this has been corrected in the RACM mechanism. The chemistry of the unsaturated dicarbonyl species and the unsaturated peroxynitrate has been revised by including $\mathrm{HO}$ and $\mathrm{NO}_{3}$ addition reactions and ozonolysis. The amount of cresol production has been reduced. For the RADM 2 mechanism, environmental chamber studies showed that the timing for the cresol chemistry was poor and that the cresol chemistry produced too much ozone. The new RACM mechanism has improved the timing for the cresol chemistry, but the level of ozone production requires additional improvement.

The peroxyacetyl nitrate chemistry and the organic peroxy radical-peroxy radical reactions were revised. Organic peroxy radical $+\mathrm{NO}_{3}$ reactions were added according to Kirchner and Stockwell [1996a]. These revisions lead to significantly lower calculated PAN concentrations.

The oxidation mechanism for isoprene was completely re- 
vised from the RADM2 mechanism. The new isoprene mechanism includes a good representation of methacrolein, and it includes treatment of isoprene ozonolysis, hydroperoxide production, and production of carbonitrates from the reaction of isoprene with $\mathrm{NO}_{3}$. The RACM mechanism includes a mechanism for the oxidation of $\alpha$-pinene and d-limonene. Tests against environmental chamber experiments show a reasonably good agreement between simulation and experiment [Kirchner and Stockwell, 1996b].

Both the RADM and RACM mechanisms reproduce the concentrations of $\mathrm{O}_{3}, \mathrm{NO}_{2}$, and hydrocarbons and the timing of peak $\mathrm{O}_{3}$ and $\mathrm{NO}_{2}$ concentrations measured in environmental chambers to within about $\pm 30 \%$. Intercomparisons for rural and urban conditions show that the RADM and RACM predict similar concentrations of $\mathrm{O}_{3}, \mathrm{H}_{2} \mathrm{O}_{2}, \mathrm{H}_{2} \mathrm{SO}_{4}$, and $\mathrm{HNO}_{3}$, but the PAN concentrations are significantly different. Because the RACM chemistry is based upon significantly more reliable laboratory measurements and evaluated rate constants, we strongly recommend this mechanism for use in regional atmospheric chemistry models.

Research on the development of tropospheric gas-phase reaction mechanisms must be considered to be a continuing process because of significant gaps and uncertainties in the laboratory kinetic data. These important gaps include several topics for research. The reactions of ozone and $\mathrm{NO}_{3}$ with alkenes and their reaction products require better characterization. For these compounds, uncertainties include the nature and yield of radicals, hydrogen peroxide, hydroperoxides, and organic acids. The oxidation mechanisms of biogenic hydrocarbons including isoprene and terpenes are very complex and poorly understood. The uncertainties in aromatic chemistry are very high; many of the products have not been characterized. The initial fate of the aromatic-HO adduct is not known. The point during the oxidation cycle where the aromatic ring breaks is not known nor is the location of the break known. Although many rate constants for the primary reactions of $\mathrm{HO}$, $\mathrm{O}_{3}$, and $\mathrm{NO}_{3}$ with organic compounds have been measured, there has been insufficient progress in the understanding of the secondary organic chemistry. Product yields and the reactions of many photooxidation products remain to be investigated. This is especially true for the chemistry of higher molecular weight organic compounds. There are few available data on the secondary chemistry for compounds with carbon numbers greater than 3 or 4 . For example, the nature, yield, and fate of many of the carbonyl products produced from the high molecular weight alkanes, alkenes and other compounds requires more laboratory data. The reactions of organic peroxy radicals with $\mathrm{NO}, \mathrm{HO}_{2}, \mathrm{RO}_{2}$, and $\mathrm{NO}_{3}$ need to be better characterized because these reactions may strongly affect the concentrations of PAN, organic nitrate, organic peroxides, $\mathrm{H}_{2} \mathrm{O}_{2}$, and ozone. For higher molecular weight alkoxy radicals the relative importance of unimolecular decomposition, reaction with oxygen, and isomerization reactions is unknown, and this may effect simulated ozone production rates.

More environmental chambcr and ficld data are required to test the mechanisms. Uncertainties in photolysis rates, wall effects, and initial conditions and the relatively high concentrations that are used in environmental chambers limit the usefulness of the data now available. Cleaner environmental experiments with a wider number of measured species are required. Field measurements suffer from uncertainties in meteorological conditions, photolysis rates, emissions, initial concentrations and air mass age, effects of heterogeneous and aqueous phase reactions, deposition rates, etc. One possible solution to the problem is comparison of in situ measurements of $\mathrm{HO}$ and other radical intermediates with model simulations based upon a reasonably complete dataset of meteorological parameters, photolysis frequencies, $\mathrm{NO}_{x}$, and VOC concentrations [Poppe et al., 1994]. These tests and similar ones should be repeated for the new mechanism. Finally, simultaneous field measurements of $\mathrm{NO}_{x}, \mathrm{PAN}$, organic nitrates, and $\mathrm{HNO}_{3}$ would be a useful test of the mechanism because the secondary peroxy radical chemistry strongly affects the partitioning between nitrogen species [Stockwell et al., 1990, 1995; Kirchner and Stockwell, 1996a].

Acknowledgments. Support for this research was provided by the German Bundesministerium für Bildung, Wissenschaft, Forschung und Technologie, projects "Spurenstoffkreisläufe," contract 07VSK01/4 and "Der Einfluss biogener Kohlenwasserstoffe auf das troposphärische Oxidationsverhalten: Abbaumechanismus biogener Alkene über Reaktionen mit $\mathrm{OH}, \mathrm{NO}_{3}$ und $\mathrm{O}_{3}$ ", and by the Commission of the European Communities, projects "Biogenic Emissions in the Mediterranean Area (BEMA)" and "Biogenic VOC Emissions and Photochemistry in the Boreal Regions of Europe (BIPHOREP)." The authors thank Karl H. Becker and colleagues at the Bergische Universität Wuppertal for helpful discussions.

\section{References}

Andersson-Sköld, Y., Updating the chemical scheme for the IVL photochemical trajectory model, IVL Rep. B 1151, Swed. Environ. Res. Inst., Götegorg, Sweden, 1995.

Atkinson, R., Gas-phase tropospheric chemistry of organic compounds: A review, Atmos. Environ., 24A, 1-41, 1990.

Atkinson, R., Kinetics and mechanisms of the gas-phase reactions of the $\mathrm{NO}_{3}$ radical with organic compounds, J. Phys. Chem. Ref. Data, 20, 459-507, 1991.

Atkinson, R., Gas-phase tropospheric chemistry of organic compounds: A review, J. Phys. Chem. Ref. Data, Monogr. 2, 1-216, 1994.

Atkinson, R., and S. M. Aschmann, OH radical production from the gas-phase reactions of $\mathrm{O}_{3}$ with a series of alkenes under atmospheric conditions, Environ. Sci. Technol., 27, 1357-1363, 1993.

Atkinson, R., and S. M. Aschmann, Products of the gas-phase reactions of aromatic hydrocarbons: Effect of $\mathrm{NO}_{2}$ concentration, Int. J. Chem. Kinet., 26, 929-944, 1994.

Atkinson, R., and A. C. Lloyd, Evaluation of kinetic and mechanistic data for modeling of photochemical smog, J. Phys. Chem. Ref. Data, 13, 315-444, 1984.

Atkinson, R., S. M. Aschmann, J. Arey, and W. P. L. Carter, Formation of ring-retaining products from the $\mathrm{OH}$ radical-initiated reactions of benzene and toluene, Int. J. Chem. Kinet., 21, 801-827, 1989.

Atkinson, R., S. M. Aschmann, and J. Arey, Formation of ringretaining products from the $\mathrm{OH}$ radical-initiated reactions of 0 -, $m-$, and $p$-xylene, Int. J. Chem. Kinet., 23, 77-97, 1991.

Atkinson, R., S. M. Aschmann, and J. Arcy, Rcactions of $\mathrm{OH}$ and $\mathrm{NO}_{3}$ radicals with phenol, cresols and 2-nitrophenol at $296 \pm 2 \mathrm{~K}$, Environ. Sci. Technol., 26, 1397-1403, 1992a.

Atkinson, R., D. L. Baulch, R. A. Cox, R. F. Hampson, J. A. Kerr, and J. Troe, Evaluated kinetic and photochemical data for atmospheric chemistry: Supplement IV, IUPAC subcommittee on gas kinetic data evaluation for atmospheric chemistry, J. Phys. Chem. Ref. Data, 21, 1125-1568, $1992 \mathrm{~b}$.

Atkinson, R., E. C. Tuazon, I. Bridier, and J. Arey, Reactions of $\mathrm{NO}_{3}$-naphthalene, adducts with $\mathrm{O}_{2}$ and $\mathrm{NO}_{2}$, Environ. Sci. Technol., 28, 605-614, 1994.

Barnes, I., V. Bastian, K. H. Becker, and T. Zhu, Kinetic and products of the reactions of $\mathrm{NO}_{3}$ with monoalkenes, dialkenes, and monoterpene, J. Phys. Chem., 94, 2413-2419, 1990.

Barnes, I., B. Klotz, and K. H. Becker, Aromatic hydrocarbon oxidation: New developments, in Proceedings of the Air \& Waste Management Association 89th Annual Meeting \& Exhibition, June 23-28, 1996, Nashville, Tennessee, Air and Waste Manage. Assoc., Pittsburgh, Pa., 1996.

Becker, E., M. M. Rahman, and R. N. Schindler, Determination of the 
rate constants for the gas phase reactions of $\mathrm{NO}_{3}$ with $\mathrm{H}, \mathrm{OH}$ and $\mathrm{HO}_{2}$ radicals at $298 \mathrm{~K}$, Ber. Bunsenges. Phys. Chem., 96, 776-783, 1992.

Becker, K. H., J. Bechara, and K. J. Brockmann, Studies on the formation of $\mathrm{H}_{2} \mathrm{O}_{2}$ on the ozonolysis of alkenes, Atmos. Environ., 27A, 57-61, 1993.

Bierbach, A., Produktuntersuchungen und Kinetik der $\mathrm{OH}$-initiierten Gasphasenoxidation aromatischer Kohlenwasserstoffe sowie ausgewählter carbonylischer Folgeprodukte, dissertation, Bergische Univ. Gesamthochschule Wuppertal, Wuppertal, Germany, 1994.

Bierbach, A., I. Barnes, K. H. Becker, and E. Wiesen, Atmospheric chemistry of unsaturated carbonyls: butendial, 4-oxo-2-pentenal, 3hexene-2,5-dione, maleic anhydride, $3 \mathrm{H}$-furan-2-one, and 5-methyl3H-furan-2-one, Environ. Sci. Technol., 28, 715-729, 1994.

Calvert, J. G., and S. Madronich, Theoretical study of the initial products of the atmospheric oxidation of hydrocarbons, J. Geophys. Res., 92, 2211-2220, 1987.

Calvert, J. G., and W. R. Stockwell, Acid Generation in the troposphere by gas phase chemistry, Environ. Sci. Technol., 17, 428A443A, 1983.

Cantrell, C. A., W. R. Stockwell, L. G. Anderson, K. L. Busarow, D. Perner, A. Schmeltekopf, J. G. Calvert, and H. S. Johnston, Kinetic Study of the $\mathrm{NO}_{3}-\mathrm{CH}_{2} \mathrm{O}$ Reaction and its possible role in nighttime tropospheric chemistry, J. Phys. Chem., 89, 139-146, 1985.

Cantrell, C. A., J. A. Davidson, A. H. McDaniel, R. E. Shetter, and J. G. Calvert, Temperature-dependent formaldehyde cross sections in the near-ultraviolet spectral region, J. Phys. Chem., 94, 3902-3908, 1990.

Carter, W. P. L., and F. W. Lurmann, Evaluation of the RADM GasPhase Chemical Mechanism, U.S. Environmental Protection Agency Cooperative Agreement CR-814558-01-0. Statewide Air Pollut. Res. Cent., Univ. of Calif., Riverside, 1989.

Carter, W. P. L., D. Luo, I. L. Malkina, D. Fitz, The University of California, Riverside Environmental Chamber Data Base for Evaluating Oxidant Mechanisms, vol. 1 and 2, Univ. of Calif., Riverside, 1995.

Chang, J. S., et al., NAPAP report 4, the Regional Acid Deposition Model and Engineering Model, Acidic Deposition: State-of-Science/ Technology, vol. 1, Emissions, Atmospheric Processes, and Deposition, cdited by P. M. Irving, 192 pp., Natl. Acid Precip. Assess. Program, Washington, D. C., 1991.

Cvetanovic, R. J., Chemical kinetic studies of atmospheric interest, paper presented at 12th International Symposium on Free Radicals, Laguna Beach, Calif., Jan. 4-9, 1976.

Cvetanovic, R. J., Evaluated chemical kinetic data for the reactions of atomic oxygen $\mathrm{O}\left({ }^{3} P\right)$ with unsaturated hydrocarbons, J. Phys. Chem. Ref. Data, 16, 261-326, 1987.

Dagaut, P., R. Liu, T. J. Wallington, and M. J. Kurylo, The gas phase reactivity of aliphatic polyethers towards $\mathrm{OH}$ radicals: Measurements and predictions, Int. J. Chem. Kinet., 21, 1173-1180, 1989.

Davidson, J. A., C. A. Cantrell, R. E. Shetter, A. H. McDaniel, and J. G. Calvert, The $\mathrm{NO}_{3}$ radical decomposition and $\mathrm{NO}_{3}$ scavenging in the troposphere, J. Geophys. Res., 95, 13,963-13,969, 1990.

Davis, H. F., B. Kim, H. S. Johnston, and Y. T. Lee, Dissociation energy and photochemistry of $\mathrm{NO}_{3}, J$. Phys. Chem., 97, 2172-2180, 1993.

DeMore, W. B., S. P. Sander, D. M. Golden, R. F. Hampson, M. J. Kurylo, C. J. Howard, A. R. Ravishankara, C. E. Kolb, and M. J. Molina, Chemical kinetics and photochemical data for use in stratospheric modeling, Eval. 11, Natl. Aeronaut. and Space Admin., Jet Propul. Lab., Calif. Inst. of Technol., Pasadena, 1994.

Finlayson-Pitts, B. J., and J. N. Pitts Jr., Atmospheric Chemistry: Fundamentals and Experimental Techniques, John Wiley, New York, 1986.

Gardner, E. P., P. D. Sperry, and J. G. Calvert, Photodecomposition of acrolein in $\mathrm{O}_{2}-\mathrm{N}_{2}$ mixtures, J. Phys. Chem., 91, 1922-1930, 1987.

Gery, M. W., G. Z. Whitten, J. P. Killus, and M. C. Dodge, A photochemical mechanism for urban and regional scale computer modeling, J. Geophys. Res., 94, 12,925-12,956, 1989.

Giguere, P. A., and A. W. Olmos, Sur le spectre ultraviolet de l'acide peracétique et l'hydrolyse des peracétates, Can. J. Chem., 34, 689691, 1956.

Grosjean, D., E. L. Williams II, and E. Grosjean, Gas phase reaction of the hydroxyl radical with the unsaturated peroxyacyl nitrate $\mathrm{CH}_{2}=\left(\mathrm{CH}_{3}\right) \mathrm{C}(\mathrm{O}) \mathrm{OONO}_{2}$, Int. J. Chem. Kinet., 25, 921-929, 1993a.

Grosjean, D., E. Grosjean, and E. L. Williams II, The reaction of ozonc with MPAN, $\mathrm{CH}_{2}=\mathrm{C}\left(\mathrm{CH}_{3}\right) \mathrm{C}(\mathrm{O}) \mathrm{OONO}_{2}$, Environ. Sci. Technol., 27, 2548-2552, 1993 b.

Hall, I. W., R. P. Wayne, R. A. Cox, M. E. Jenkin, and G. D. Hayman, Kinetics of the reaction of $\mathrm{NO}_{3}$ with $\mathrm{HO}_{2}$, J. Phys. Chem., 92, 50495054, 1988.

Hass, H., H. J. Jakobs, and M. Memmesheimer, Analysis of a regional model (EURAD) near surface gas concentration predictions using observations from networks, Meteorol. Atmos. Phys., 57, 173-200, 1995.

Hjorth, J., C. Lohse, C. J. Nielsen, H. Skov, and G. Restelli, Products and mechanisms of the gas phase reactions between $\mathrm{NO}_{3}$ and a series of alkenes, J. Phys. Chem., 94, 7494-7500, 1990.

Hjorth, J., J. Notholt, and G. Restelli, A spectroscopic study of the equilibrium $\mathrm{NO}_{2}+\mathrm{NO}_{3}+\mathrm{M} \leftrightarrow \mathrm{N}_{2} \mathrm{O}_{5}+\mathrm{M}$ and the kinetics of the $\mathrm{O}_{3} / \mathrm{N}_{2} \mathrm{O}_{5} / \mathrm{NO}_{3} / \mathrm{NO}_{2}$ /air system, Int. J. Chem. Kinet., 24, 51-65, 1992.

Horie, O., and G. K. Moortgat, Decomposition pathways of the excited Criegee intermediates in the ozonolysis of simple alkenes, Atmos. Environ., 25A, 1881-1896, 1991.

Japar, S. M., and H. Niki, Gas-phase reactions of the nitrate radical with olefins, J. Phys. Chem., 79, 1629-1632, 1975.

Jenkin, M. E., S. M. Saunders, and M. J. Pilling, Constructing a detailed chemical mechanism for use in tropospheric chemistry models, report, AEA Technol., Natl. Environ. Technol. Cent., Culham, England, 1996.

Johnston, H. S., C. A. Cantrell, and J. G. Calvert, Unimolecular decomposition of $\mathrm{NO}_{3}$ to form $\mathrm{NO}$ and $\mathrm{O}_{2}$ and a review of $\mathrm{N}_{2} \mathrm{O}_{5} / \mathrm{NO}_{3}$ kinetics, J. Geophys. Res., 91, 5159-5172, 1986.

Joseph, J. H., W. J. Wiscombe, and J. A. Weinman, The deltaEddington approximation for radiative flux transfer, J. Atmos. Sci. 33, 2452-2458, 1976.

Kirchner, F. and W. R. Stockwell, Effect of peroxy radical reactions on the predicted concentrations of ozone, nitrogenous compounds, and radicals, J. Geophys. Res., 101, 21,007-21,022, 1996a. (Correction to "Effect of peroxy radical reactions on the predicted concentrations of ozone, nitrogenous compounds and radicals" by F. Kirchner and W. R. Stockwell, J. Geophys. Res., in press, 1997.)

Kirchner, F., and W. R. Stockwell, Isoprene and terpene oxidation mechanisms, in Proceedings of the EUROTRAC Symposium '96, Transport and Transformation of Pollutants in the Troposphere, Volume 1: Clouds, Aerosols, Modelling and Photooxidants, edited by P. M. Borrell et al., pp. 715-719, Comput. Mech. Publ., Southampton, England, 1996b.

Knispel, R., R. Koch, M. Siese, and C. Zetzsch, Adduct formation of $\mathrm{OH}$ radicals with benzene, toluene and phenol and consecutive reactions of the adducts with $\mathrm{NO}_{x}$ and $\mathrm{O}_{2}$, Ber. Bunsenges. Phys. Chem., 94, 1375-1379, 1990.

Koch, S., and G. K. Moortgat, Photolysis of methylgloxyal, in Air Pollution Research Report Series Research Report 57, Homogeneous and Heterogeneous Chemical Processes in the Troposphere, Joint Workshop LACTOZ-HALIPP, Septernber 25-26, Strasbourg, France, pp. 327-331, Off. for Off. Publ. of the Eur. Commun., Luxembourg, 1996.

Kwok, E. S. C., and R. Atkinson, Estimation of hydroxyl radical rate constants for gas phase organic compounds using a structurereactivity relationship: An update, Atmos. Environ., 29, 1685-1695, 1995.

Le Bras, G. (Ed), Chemical Processes in Atmospheric Oxidation, Springer-Verlag, New York, 1997.

Leone, J. A., and J. H. Seinfeld, Comparative analysis of chemical reaction mechanisms for photochemical smog, Atmos. Environ., 19, 437-464, 1985

Lightfoot, P. D., R. A. Cox, J. N. Crowley, M. Destriau, G. D. Haymạn, M. E. Jenkin, G. K. Moortgat, and F. Zabel, Organic peroxy radicals: Kinetics, spectroscopy and tropospheric chemistry, Atmos. Environ., 26A, 1806-1963, 1992.

Lurmann, F. W., A. C. Lloyd, and R. Atkinson, A chemical mechanism for use in long-range transport/acid deposition computer modeling, J. Geophys. Res., 91, 10,905-10,936, 1986.

Madronich, S., Photodissociation in the atmosphere, 1, Actinic flux and the effects of ground reflections and clouds, J. Geophys. Res., 92, 9740-9752, 1987.

Martinez, R. D., A. A. Buitrago, N. W. Howell, C. H. Hearn, and J. A. Joens, The near U.V. absorption spectra of several aliphatic aldehydes and ketones at $300 \mathrm{~K}$, Atmos. Environ., 26A, 785-792, 1992.

McRae, G. J., W. R. Gooden, and J. H. Seinfeld, Mathematical Mod- 
eling of Photochemical Air Pollution, EQL Rep. 18, Environ. Qual. Lab., Calif. Inst. of Technol., Pasadena, 1982.

Mellouki, A., G. Le Bras, and G. Poulet, Kinetics of the reactions of $\mathrm{NO}_{3}$ with $\mathrm{OH}$ and $\mathrm{HO}_{2}$, J. Phys. Chem., 92, 2229-2234, 1988.

Mellouki, A., R. K. Talukdar, A. M. R. P. Bopegedera, and C. J. Howard, Study of the kinetics of the reactions of $\mathrm{NO}_{3}$ with $\mathrm{HO}_{2}$ and OH, Int. J. Chem. Kinet., 25, 25-39, 1993.

Middleton, P., W. R. Stockwell, and W. P. L. Carter, Aggregation and analysis of volatile organic compound emissions for regional modeling, Atmos. Environ., 24A, 1107-1133, 1990.

Moortgat, G. K., W. Klippel, K. H. Mobus, W. Seiler, and P. Warncck, Laboratory measurement of photolytic parameters for formaldehyde, Final Rep. FAA-EE-80-47, Off. of Environ. and Energy, Fed. Aviat. Admin., Washington, D. C., 1980.

Moortgat, G. K., et al., Laboratory studies of peroxy radicals, carbonyl compounds and ozonolysis reactions of tropospheric importance, in Chemical Processes in Atmospheric Oxidation, edited by G. Le Bras, pp. 162-169, Springer-Verlag, New York, 1997.

Morris, E. D., Jr., and H. Niki, Reaction of the nitrate radical with acetaldehyde and propylene, J. Phys. Chem., 78, 1337-1338, 1974.

National Center for Atmospheric Research (NCAR) Preliminary Evaluation Studies with the Regional Acid Deposition Model (RADM), NCAR Tech. Note NCAR/TN-265+STR, Natl. Cent. for Atmos. Res., Boulder, Colo., 1986.

Paulson, S. E., R. C. Flagan, and J. H. Seinfeld, Atmospheric photooxidation of isoprene, I, The hydroxyl radical and ground state atomic oxygen reactions, Int. J. Chem. Kinet., 24, 79-101, 1992a.

Paulson, S. E., R. C. Flagan, and J. H. Seinfeld, Atmospheric photooxidation of isoprene, II, The ozone-isoprene reaction, Int. J. Chem. Kinet., 24, 103-125, $1992 \mathrm{~b}$.

Platt, U., D. Perner, A. M. Winer, G. W. Harris, and J. N. Pitts Jr., Detection of $\mathrm{NO}_{3}$ in the polluted troposphere by differential optical absorption, Geophys. Res. Lett., 7, 89-92, 1980.

Poppe, D., et al., Comparison of measured $\mathrm{OH}$ concentrations with model calculations, J. Geophys. Res., 99, 16,633-16,642, 1994.

Poppe, D., et al., Gas-phase reactions in atmospheric chemistry and transport models: A model intercomparison, report, EUROTRAC Int. Sci. Secr., Garmisch-Partenkirchen, Germany, 1996.

Russell, A. G., G. R. Cass, and J. H. Seinfeld, On some aspects of nighttime atmospheric chemistry, Environ. Sci. Technol., 20, 1167$1172,1986$.

Seinfeld, J. H., Atmospheric Chemistry and Physics of Air Pollution, John Wiley, New York, 1986.

Skov, H., J. Hjorth, C. Lohse, C. J. Jensen, and G. Restelli, Products and mechanisms of the reactions of the nitrate radical $\left(\mathrm{NO}_{3}\right)$ with isoprene, 1,3-butadien and 2,3-dimethyl-1,3-butadien in air, Atmos. Environ., 26A, 2771-2783, 1992.

Staffelbach, T. A., J. J. Orlando, G. S. Tyndall, and J. G. Calvert, The UV-visible absorption spectrum and photolysis quantum yields of methylglyoxal, J. Geophys. Res., 100, 14,189-14,198, 1995.

Stockwell, W. R., and J. G. Calvert, The mechanism of $\mathrm{NO}_{3}$ and HONO formation in the nighttime chemistry of the urban atmosphere, J. Geophys. Res., 88, 6673-6682, 1983.

Stockwell, W. R., and D. Kley, The Euro-RADM Mechanism: A gasphase chemical mechanism for European air quality studies, Forschungszentrum, Jülich, Jülich, Germany, 1994.

Stockwell, W. R., and F. W. Lurmann, Intercomparison of the ADOM and RADM gas-phase chemical mechanisms, report, Electr. Power Res. Inst., Palo Alto, Calif., 1989.
Stockwell, W. R., P. Middleton, J. S. Chang, and X. Tang, The second generation regional Acid Deposition Model chemical mechanism for regional air quality modeling, J. Geophys. Res., 95, 16,343-16,367, 1990.

Stockwell, W. R., J. B. Milford, D. Gao, and Y.-J. Yang, The effect of acetyl peroxy-peroxy radical reactions on peroxyacetyl nitrate and ozone concentrations, Atmos. Environ., 29, 1591-1599, 1995.

Sverdrup, G. M., C. W. Spicer, and G. F. Ward, Investigation of the gas phase reaction of dinitrogen pentoxide with water vapor, Int. J. Chem. Kinet., 19, 191-205, 1987.

Tuazon, E. C., and R. Atkinson, A product study of the gas phase reaction of methacrolein with the $\mathrm{OH}$ radical in the presence of $\mathrm{NO}_{x}$, Int. J. Chem. Kinet., 22, 591-602, 1990.

Tuazon, E. C., R. Atkinson, and W. P. L. Carter, Atmospheric chemistry of cis- and trans-3-hexene-2,5-dione, Environ. Sci. Technol., 19, 265-269, 1985.

Tuazon, E. C., J. Arey, R. Atkinson, and S. M. Aschmann, Gas-phase reactions of 2-vinylpyridine and styrene with $\mathrm{OH}$ and $\mathrm{NO}_{3}$ radicals and $\mathrm{O}_{3}$, Environ. Sci. Technol., 27, 1832-1841, 1993.

Uselman, W. M., S. Z. Levine, W. H. Chan, J. G. Calvert, and J. H. Shaw, A kinetic study of the mechanism of peroxynitric acid formation in irradiated mixtures of chlorine, hydrogen, nitrogen dioxide and nitric oxide in air, in Nitrogenous Air Pollutants, Chemical and Biological Implications, edited by D. Grosjean, Ann Arbor Sci., Ann Arbor, Mich., 1979.

Vogel, B., F. Fiedler, and H. Vogel, Influence of topography and biogenic volatile organic compounds emission in the state of BadenWürttemberg on ozone concentrations during episodes of high air temperatures, J. Geophys. Res., 100, 22,907-22,928, 1995.

Wallington, T. J., P. Dagaut, R. Liu, and M. J. Kurylo, The gas-phase reactions of hydroxyl radicals with a series of esters over the temperature range 240-440 K, Int. J. Chem. Kinet., 20, 177-186, 1988.

Warneck, P., Chemistry of the Natural Atmosphere, Academic, San Diego, Calif., 1988.

Wayne, R. P., et al., The nitrate radical: Physics, chemistry, and the atmosphere, Atmos. Environ., 25A, 1-203, 1991.

Wiesen, E., OH-initiierte Oxidation von p-Xylol und Produktuntersuchungen an ausgewaehlten Carbonylverbindungen (1995), dissertation, Bergische Univ. Gesamthochschule Wuppertal, Wuppertal, Germany, 1995.

Wiesen, E., I. Barnes and K. H. Becker, Study of the $\mathrm{OH}$-initiated degradation of the aromatic photooxidation product 3,4-dihydroxy3-hexene-2,5-dione, Environ. Sci. Technol., 29, 1380-1386, 1995.

Williams, D. C., L. N. O'Rji, and D. A. Stone, Kinetics of the reactions of $\mathrm{OH}$ radicals with selected acetates and other esters under simulated atmospheric conditions, Int. J. Chem. Kinet., 25, 539-548, 1993.

F. Kirchner, M. Kuhn, and W. R. Stockwell, Fraunhofer Institute for Atmospheric Environmental Research, Kreuzeckbahnstr. 19, D-82467 Garmisch-Partenkirchen, Germany. (e-mail: stockwel@ifu.fhg.de)

S. Seefeld, EAWAG, Swiss Federal Institute for Environmental Science and Technology, ETH Zürich, CH-8600 Dübendorf, Switzerland.

(Received December 6, 1996; revised March 17, 1997; accepted March 17, 1997.) 